\title{
ATLAS DE DISTRIBUCIÓN Y ESTADO DE CONSERVACIÓN DE LOS ANFIBIOS DE LA COMUNIDAD DE MADRID
}

\author{
I. Martínez-Solano ${ }^{1,2, *}$
}

\begin{abstract}
RESUMEN
La planificación de estrategias de gestión y conservación eficaces depende de la disponibilidad, integración y actualización de información procedente de diversas disciplinas. En este trabajo se recopila información relevante para la conservación de los anfibios de la Comunidad de Madrid. Hemos obtenido información corológica de un total de 1384 puntos de muestreo repartidos por toda la geografía madrileña y revisado la literatura científica de interés para la gestión de las poblaciones de anfibios en esta región. En el 70,3\% de los puntos muestreados se ha encontrado al menos una especie de anfibio. Los resultados se ilustran mediante mapas de distribución de cada una de las 18 especies encontradas: Salamandra salamandra, Pleurodeles waltl, Mesotriton alpestris, Lissotriton boscai, Triturus marmoratus, Triturus pygmaeus, Alytes obstetricans, Alytes cisternasii, Discoglossus galganoi, Discoglossus jeanneae, Pelobates cultripes, Pelodytes punctatus, Hyla arborea, Hyla meridionalis, Bufo bufo, Bufo calamita, Rana iberica y Rana perezi. Los mapas de distribución se presentan en cuadrículas UTM de $5 \times 5 \mathrm{~km}$. Se comparan e integran los resultados obtenidos con los de estudios anteriores y se delimitan grupos de poblaciones que representan unidades independientes de gestión, para los cuales se proponen categorías de amenaza según los criterios establecidos por la Unión Internacional para la Conservación de la Naturaleza (IUCN) a nivel regional. Finalmente, se señalan las principales amenazas para la supervivencia de las poblaciones de anfibios madrileños y se discuten posibles actuaciones para mejorar su estado de conservación.

Palabras clave: Distribución, Conservación, Anfibios, España, Madrid, Categorías
\end{abstract} IUCN.

\section{ABSTRACT \\ Distribution atlas and conservation status of amphibians in Comunidad de Madrid}

Successful management and conservation planning depend upon the availability, integration and updating of information provided by different disciplines. This paper compiles relevant information for the conservation of amphibians in Comunidad de Madrid (Spain). We obtained chorological information from 1384 sampling localities throughout the region of Madrid, and reviewed relevant scientific literature for the management of

Museo Nacional de Ciencias Naturales, CSIC. c/ José Gutiérrez Abascal, 2. 28006 Madrid, España.

Museum of Vertebrate Zoology, University of California, Berkeley. 3101 Valley Life Sciences Building. 94720-3160 Berkeley, California, USA.

email: inigomsolano@berkeley.edu 
amphibian populations in this area. We found at least one amphibian species in $70,3 \%$ of sampled localities. Results are illustrated in distribution maps of all 18 species found: Salamandra salamandra, Pleurodeles waltl, Mesotriton alpestris, Lissotriton boscai, Triturus marmoratus, Triturus pygmaeus, Alytes obstetricans, Alytes cisternasii, Discoglossus galganoi, Discoglossus jeanneae, Pelobates cultripes, Pelodytes punctatus, Hyla arborea, Hyla meridionalis, Bufo bufo, Bufo calamita, Rana iberica and Rana pere$z i$. Distribution maps are presented in five-kilometer side UTM grids cells. We compare and integrate our results with previous studies and outline groups of populations representing independent management units, for which we propose risk categories according to the criteria established by the International Union for the Conservation of Nature (IUCN) at the regional level. Finally, we point out the main threats for the survival of amphibian populations and discuss possible actions to improve their conservation status.

Key words: Distribution, Conservation, Amphibians, Spain, Madrid, IUCN Categories.

\section{Introducción}

Una de las primeras etapas en el desarrollo de cualquier proyecto relacionado con la gestión y conservación de los recursos naturales debe consistir en la elaboración de inventarios. Conocer de qué recursos se dispone y cómo se reparten éstos sobre el territorio resulta fundamental para poder elaborar estrategias de gestión. Además, a la dimensión espacial de la distribución de los recursos naturales se superpone una componente temporal que debe ser también considerada en los planes de gestión. Las poblaciones animales pueden variar su disposición sobre el territorio, muchas veces a lo largo de escalas temporales muy reducidas, en función de aspectos demográficos, bien expandiéndose colonizando nuevos territorios, o bien, como ocurre más frecuentemente, fragmentándose o extinguiéndose en respuesta a diversos factores negativos, incluida la acción humana. Por ello, la actualización regular de los inventarios faunísticos resulta fundamental, ya que permite detectar tendencias demográficas, como expansiones o declives (ver, por ejemplo, los resultados preliminares del Proyecto Grinnell, desarrollado por la Universidad de California, Berkeley, en la web: http://mvz.berkeley.edu/Grinnell/index.html), y planificar en consecuencia la estrategia de gestión más apropiada en cada caso (por ejemplo, mediante la selección de taxones o áreas geográficas cuya protección resulta prioritaria).

Los anfibios constituyen un grupo amenazado a escala global (Stuart et al., 2004), lo que ha motivado una creciente preocupación acerca de su estado de conservación. Las causas del declive de los anfibios parecen residir en la interacción entre numerosos factores negativos (Alford \& Richards, 1999; Kiesecker et al., 2001; Pounds et al., 2006). En áreas dominadas por la influencia humana, el factor preponderante suele ser la pérdida de hábitats favorables, ya sea a través de su alteración (especialmente la contaminación de los medios acuáticos o la introducción de especies exóticas) o por su destrucción directa (Lizana \& Barbadillo, 1997). La consecuencia inmediata es la fragmentación de las poblaciones, un factor crítico ya que su viabilidad en un contexto regional depende de que se mantenga un cierto grado de conectividad entre ellas (Cushman, 2006). Por todo ello, disponer de información detallada acerca de los patrones de distribución espacial de las poblaciones de anfibios resulta indispensable para evaluar su viabilidad $\mathrm{y}$, en consecuencia, planificar su gestión de manera eficaz.

El objetivo de este trabajo es ofrecer información actualizada acerca de la distribución de las poblaciones de anfibios de la Comunidad de Madrid y realizar una evaluación de su estado de conservación, incluida una propuesta de catalogación de cada especie de acuerdo con las categorías de la Unión Internacional para la Conservación de la Naturaleza (IUCN). Para ello se ha recopilado la información corológica y genética disponible, con el fin de delimitar dentro de cada especie grupos de poblaciones que formen unidades coherentes desde un punto de vista evolutivo y sirvan de ayuda a la hora de planificar la gestión de cada especie a nivel regional.

En cuanto a la información corológica, ya se han publicado con anterioridad mapas de distribución de los anfibios madrileños (García París et al., 1989). Sin embargo, en los años posteriores a la publicación de ese trabajo tan sólo se ha añadido información puntual sobre algunas zonas en informes inéditos o notas breves de distribución (Álvarez-Vasserot, 1991; Grijalbo, 1991; Prieto, 1991; García-Avilés et al., 1999; Aceituno, 2001; 
Martínez-Solano \& García-París, 2001; RecueroGil et al., 2002). Por ello, se ha completado esta información con la obtenida durante nuevos muestreos realizados durante los años 1999 a 2004.

Por otro lado, durante los últimos años se han completado varios estudios que han ofrecido información relevante para la conservación de las poblaciones de anfibios madrileñas desde diversas perspectivas (demográfica, ecológica, genética, taxonómica) (García-París \& Jockusch, 1999; Bosch et al., 2001; García-París \& MartínezSolano, 2001; García París et al., 2001, 2003; Gómez-Mestre \& Tejedo, 2003, Martínez-Solano et al., 2003; Martínez-Solano, 2004; Martínez-Solano et al., 2004, 2005a,b; Real et al., 2005; Bosch et al., 2006). Por tanto, resulta oportuno hacer una síntesis de la información disponible hasta el momento, llevar a cabo una revisión y actualización de ésta y completarla en los casos en que resulte necesario.

\section{Material y métodos}

Las citas empleadas para la elaboración del Atlas de los Anfibios de Madrid proceden de diversas fuentes, que incluyen citas bibliográficas, revisión de colecciones científicas (ver Martínez-Solano \& González-Fernández, 2003), citas aportadas por particulares y datos propios obtenidos en muestreos recientes.

Las citas bibliográficas proceden de los siguientes trabajos: García-París et al. (1989, 1990); Álvarez-Vasserot (1991); Grijalbo (1991); Prieto (1991); Astudillo et al. (1993); Lizana et al. (1995); De la Riva et al. (1998); Bosch et al. (1998, 2000); García-Avilés et al. (1999), Martínez-Solano \& García-París (2001); Aceituno (2001); SCV (2001a); Recuero-Gil et al. (2002); Pleguezuelos et al. (2002). En todos los casos se ha tratado de localizar con la mayor precisión posible las localidades muestreadas y confirmar las citas en muestreos más recientes. Como ayuda se ha contado con las fichas originales que incluyen las citas del Atlas provisional de los Anfibios y Reptiles de Madrid (García-París et al., 1989), y con la base de datos que recoge las citas incluidas en el recientemente publicado Atlas y Libro Rojo de los Anfibios y Reptiles de España (Pleguezuelos et al., 2002). En ciertos casos ha sido posible consultar directamente a los autores de las citas acerca de las localidades muestreadas.

Las nuevas citas proceden en su mayor parte de muestreos propios llevados a cabo entre los años 1999 y 2004 . Estos muestreos han consistido habi- tualmente en la selección previa de áreas de potencial interés mediante la consulta de la cartografía disponible y la posterior visita de los lugares seleccionados. Las visitas se llevaron a cabo tanto durante el día como de noche. En el primer caso se buscaron ejemplares adultos ocultos bajo refugios como troncos o piedras, y también se realizaron muestreos de larvas y adultos mediante el empleo de salabres. Durante la noche se realizaron varios tipos de muestreo. En primer lugar, se realizaron muestreos acústicos en las inmediaciones de los puntos de agua. Además, se realizaron muestreos visuales en los medios acuáticos durante el periodo reproductor, generalmente transectos en los puntos de reproducción localizados durante las visitas diurnas. También se realizaron recorridos tanto diurnos como nocturnos por carretera con el fin de observar ejemplares activos o registrar la presencia de ejemplares atropellados.

En cuanto al esfuerzo de muestreo realizado, se ha tenido en cuenta las características específicas del trabajo para definir una medida apropiada. Las medidas de esfuerzo de muestreo empleadas habitualmente en la elaboración de inventarios (por ejemplo, el tiempo empleado por número de investigadores por unidad espacial muestreada) tienen como objeto estandarizar los muestreos con objeto de evaluar su repetibilidad y facilitar las comparaciones con futuros muestreos. Sin embargo, dado que nuestro objetivo era proporcionar para cada unidad espacial muestreada un listado de especies lo más exhaustivo posible, se ha considerado más conveniente dar menor importancia a igualar el esfuerzo entre diferentes áreas. Ello es debido a que en casos como el que nos ocupa, cuya batracofauna está relativamente bien conocida y tiene un número moderado de especies por cuadrícula, resulta aconsejable hacer un esfuerzo suplementario en algunas zonas con objeto de obtener unos resultados que reflejen lo más fielmente posible la composición específica exhaustiva del área muestreada. Por ello, hemos considerado más práctico definir un nivel mínimo de esfuerzo a realizar en cada una de las cuadrículas de $10 \times 10 \mathrm{~km}$ muestreadas, tratando de asegurar que la probabilidad de que se encuentren más especies si se realiza un esfuerzo mayor sea razonablemente pequeña. Este esfuerzo sería ampliable en caso de que se pudiera inferir que alguna especie pudiera haber pasado inadvertida. La necesidad de realizar un esfuerzo de muestreo adicional se ha decidido en el contexto de los resultados observados en cuadrículas vecinas. Se ha considerado que un esfuerzo de muestreo de 5 cuadrículas UTM de 1x1 km diferentes dentro de cada 
cuadrícula UTM de 10×10 km es suficiente para valorar la necesidad o no de realizar un esfuerzo adicional en cada caso particular.

Aunque la mayor parte de la información recopilada se ha referenciado a una escala geográfica de $1 x 1 \mathrm{~km}$, se ha decidido presentar los resultados en forma de mapas de distribución elaborados a una escala geográfica de $5 \times 5 \mathrm{~km}$. En este caso sólo se ha incluido la información que pudiera ser referenciada con una precisión de $1 \mathrm{x} 1 \mathrm{~km}$ y estuviera además confirmada recientemente (las demás citas, incluidas las bibliográficas y las procedentes de colecciones, se muestran también en los mapas, pero a una escala de 10x10 km). Estos mapas de distribución representan probablemente de manera más fiel características de las poblaciones de anfibios que resultan relevantes para su gestión, como su grado de conectividad, ya que distancias mayores de $5 \mathrm{~km}$ probablemente exceden la capacidad media de dispersión de la mayor parte de las especies de anfibios presentes en la Comunidad de Madrid. Como se ha comentado con anterioridad, no se han incorporado a estos mapas las citas bibliográficas que no han podido ser confirmadas, bien por la imposibilidad de localizarlas con esa resolución o bien porque los muestreos llevados a cabo han resultado negativos en ese aspecto. Estos matices se discuten en los apartados correspondientes a cada especie. El interés fundamental de incorporar y discutir esta información radica en la posibilidad de realizar comparaciones directas con los publicados por García-París et al. (1989).

La asignación de categorías de riesgo de extinción es una de las bases que deben sustentar la designación de prioridades para la conservación, proceso en el que, además, se toman en consideración factores adicionales de índole no siempre biológica, como aspectos económicos, culturales, etc. En este contexto, los criterios establecidos por la IUCN, cuyo objetivo es clasificar a los taxones que son objeto de estudio en relación con el riesgo de extinción que afrontan a corto, medio o largo plazo, son los más empleados en la elaboración de listados de especies amenazadas.

En el vigente "Catálogo Regional de Especies Amenazadas de la Comunidad de Madrid", cuya categorización no se basa en la aplicación de los criterios IUCN, una especie está catalogada como "En Peligro de Extinción" (Triturus alpestris); otra se considera "De Interés Especial" (Triturus boscai), y finalmente, tres están catalogadas como "Vulnerable" (Pelodytes punctatus; Hyla arborea y Rana iberica). Existe una propuesta más reciente de revisión de este Catálogo que sí aplica los crite- rios de la IUCN (De la Riva et al., 1998), en la que se incluyen taxones a nivel subespecífico, de acuerdo con la información disponible en aquel momento. Según esta propuesta (se indican en cada caso los criterios seguidos en la catalogación, ver IUCN, 1994), un único taxon debería ser catalogado como "En Peligro Crítico" (Alytes obstetricans pertinax: A1, B1, B2, C1); tres como "En Peligro": (Salamandra salamandra bejarae: A2, B1; Triturus alpestris cyreni: B1 y Alytes obstetricans almogavarii: A1, A2); cinco como "Vulnerable" (Salamandra salamandra almanzoris: B1; Triturus boscai: A2, B1; Triturus marmoratus: B1, B2; Bufo bufo: A2; Rana iberica: B1, B2); y cinco como "Menor Riesgo, casi amenazado" (Pleurodeles waltl; Triturus pygmaeus; Alytes cisternasii; Pelobates cultripes e Hyla arborea). Tres especies fueron catalogadas como "Datos Insuficientes": Discoglossus galganoi, Discoglossus jeanneae y Pelodytes punctatus. Esta nueva propuesta no ha sido aún puesta en práctica, de manera que la nueva información recogida en este trabajo permitiría complementarla eficazmente, como se detalla más adelante.

Uno de los objetivos de este trabajo es desarrollar una nueva propuesta que incorpore los nuevos datos corológicos y moleculares recopilados durante la elaboración de este estudio y aplique la versión más reciente de las categorías de amenaza y de los criterios de clasificación establecidos por la IUCN (IUCN, 2001).

La aplicación de las categorías definidas por la IUCN, inicialmente definidas para su uso en el ámbito global, en un contexto regional requiere la consideración del grado de conexión entre las poblaciones del taxon a evaluar en la región estudiada y las poblaciones de las regiones inmediatamente adyacentes. La base teórica supone que si existiera un flujo constante de migración entre las poblaciones de nuestra región de estudio y las cercanas, puede producirse un "efecto rescate", de manera que la probabilidad de extinción se reduce. Por ello se recomienda en estos casos evaluar primero la categoría de amenaza a nivel global y luego aplicar la siguiente que indique un riesgo menor de extinción. Por el contrario, en el caso de que el taxon objeto de estudio se encuentre aislado, sin poblaciones circundantes, el riesgo de extinción es mayor y por tanto se recomienda aplicar una categoría más restrictiva que la derivada de un análisis global (Gardenförs, 2001; Gardenförs et al., 2001a, b).

Por el momento no existen directrices claras para determinar la existencia o no de continuidad geográfica entre poblaciones localizadas, por ejemplo, 
en distintos países o entre provincias dentro de un mismo país, por lo que la aplicación de este criterio no siempre está exenta de subjetividad (ver discusión en Keller et al., 2005). Esta cuestión es relevante debido a las diferencias en cuanto a la capacidad de dispersión entre diferentes grupos taxonómicos. En nuestro caso hemos considerado que una categorización basada en criterios cuantitativos (aunque su aplicación no esté exenta de arbitrariedades) podría permitir una aplicación más rigurosa de este criterio de continuidad. Por ello, se ha cuantificado el número de cuadrículas UTM de $10 \times 10 \mathrm{~km}$ a través de las cuales se puede inferir continuidad en la distribución de cada taxon en Madrid $\mathrm{y}$ las provincias adyacentes y se ha aplicado el siguiente criterio: para aquellos taxones en los que la continuidad se limita a 5 cuadrículas o menos se propone aumentar la categoría de amenaza; para aquellas comprendidas entre 6 y 10 se propone mantener la categoría de amenaza; y finalmente, para las que presentan continuidad a través de más de 10 cuadrículas se propone rebajar la categoría de amenaza. La información corológica de las provincias circundantes (Ávila, Segovia, Guadalajara, Cuenca y Toledo), así como la referida a las categorías de amenaza de cada especie a nivel nacional procede fundamentalmente de las datos recogidos en el Atlas y Libro Rojo de los Anfibios y Reptiles de España (Pleguezuelos et al., 2002).

Siguiendo estas directrices, en este apartado se ofrecerá por un lado la categoría IUCN de cada taxon a nivel específico o, siempre que exista información al respecto, subespecífico. Por otro lado y sobre la base de esta categorización y considerando información adicional en cada caso, se hará una propuesta de prioridades de conservación para los anfibios presentes en la Comunidad de Madrid. La información adicional se detalla en cada caso particular e incluye: marginalidad (definida en este caso como el porcentaje de la superficie total de la Comunidad de Madrid ocupada por cada taxon), particularidades biológicas de las poblaciones madrileñas y finalmente, la tendencia general de las poblaciones madrileñas durante los últimos años. Por último, de acuerdo con las prioridades establecidas, se enumeran algunas propuestas de actuación en cada caso concreto.

\section{Resultados}

En total se ha obtenido información de 1384 puntos de muestreo (ver Apéndice), de los cuales 973 presentaban alguna especie de anfibio (70,3\%).
En cuanto al grado de cobertura, se ha encontrado alguna especie de anfibio en 111 cuadrículas de $10 \times 10 \mathrm{~km}(99,1 \%$ del total) y en 329 cuadrículas de $5 \times 5 \mathrm{~km}(73,43 \%$ del total $)$.

ESPECIES PRESENTES. - Se han localizado un total de 18 especies de anfibios. Con respecto al Atlas provisional de los anfibios y reptiles de Madrid (García-París et al., 1989), se han incorporado las siguientes especies: Triturus pygmaeus (anteriormente considerado conjuntamente con $T$. marmoratus), Discoglossus jeanneae (anteriormente considerado conjuntamente con $D$. galganoi) e Hyla meridionalis, cuya área de distribución penetra marginalmente en la Comunidad de Madrid. A continuación se comenta en detalle la información recogida acerca del área de distribución y estado de conservación de cada especie (se ha seguido la revisión taxonómica de García-París et al., 2004 para la denominación de cada taxon).

Salamandra salamandra (Linnaeus, 1758) (Fig. 1A)

Presente en 27 cuadrículas UTM de 10x10 km (43 cuadrículas de $5 \times 5 \mathrm{~km}$ ). Se han encontrado poblaciones de S. salamandra entre 640 y 2200 metros de altitud. Con respecto a las citas bibliográficas, no se han vuelto a observar salamandras en las inmediaciones de Somosierra y Puebla de la Sierra (UTM VL-45 y 55) ni tampoco en Navafría (VL-34), si bien en este último caso la proximidad de otras poblaciones (Puerto de Navafría, Lozoya) sugiere que nuevas prospecciones podrían ofrecer resultados positivos, aunque en todo caso estas poblaciones serían escasas.

Los mapas de distribución muestran la existencia de dos grupos de poblaciones disjuntos. El primero ocupa las zonas de baja altitud junto a los valles de los ríos Tiétar y Alberche. El segundo núcleo se extiende por las zonas más altas de la Sierra del Guadarrama, desde El Escorial hasta el Puerto de Peña Quemada, en Braojos. Estas poblaciones son cada vez más dispersas en sentido oeste-este. Los datos de ADN mitocondrial, por otro lado, muestran también la existencia de dos linajes bien diferenciados (Martínez-Solano et al., 2005a), pero en este caso los límites entre grupos son diferentes. Así, las poblaciones localizadas en la Sierra de la Higuera, en Cenicientos, son más similares genéticamente a las de la Sierra del Guadarrama que a las de los municipios más cercanos de Pelayos de la Presa, Robledo de Chavela, El Tiemblo o La Adrada. Las primeras han sido asignadas a la subespecie $S$. $S$. 
A

Salamandra salamandra

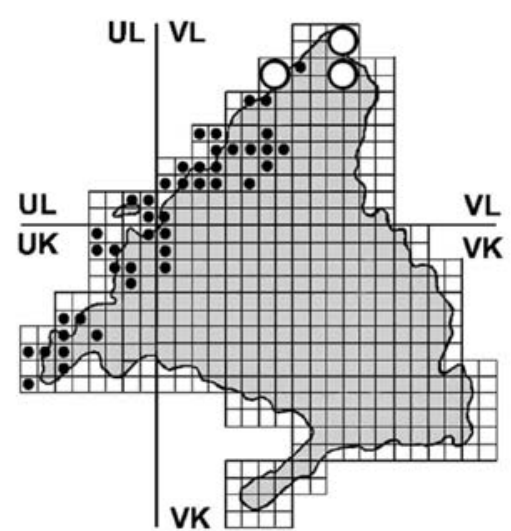

C Mesotriton alpestris

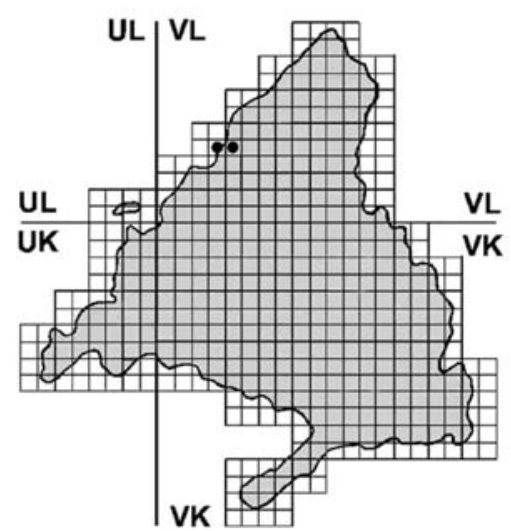

E

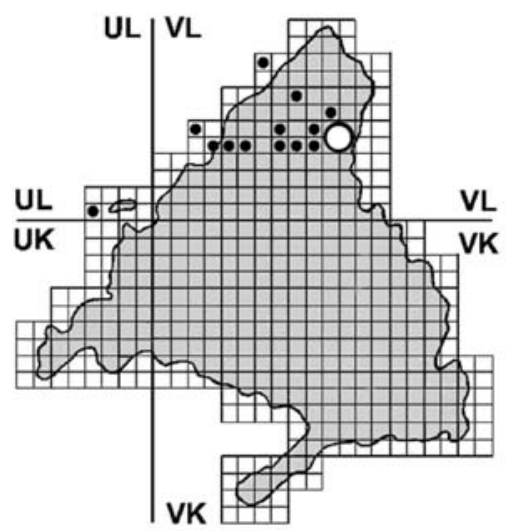

B

Pleurodeles waltl

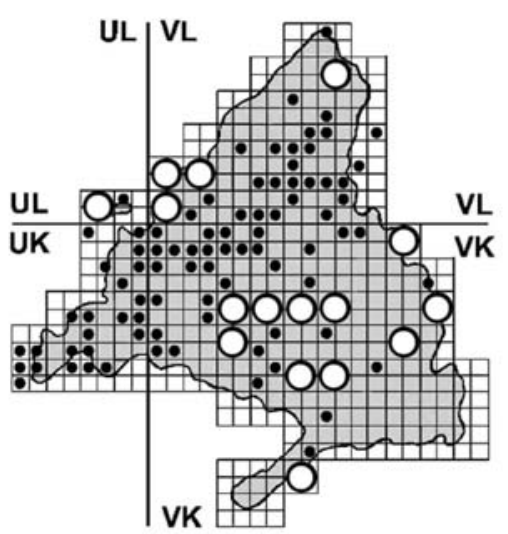

D

Lissotriton boscai

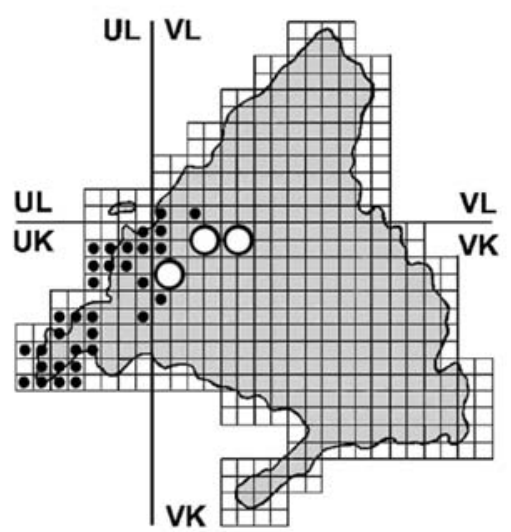

F

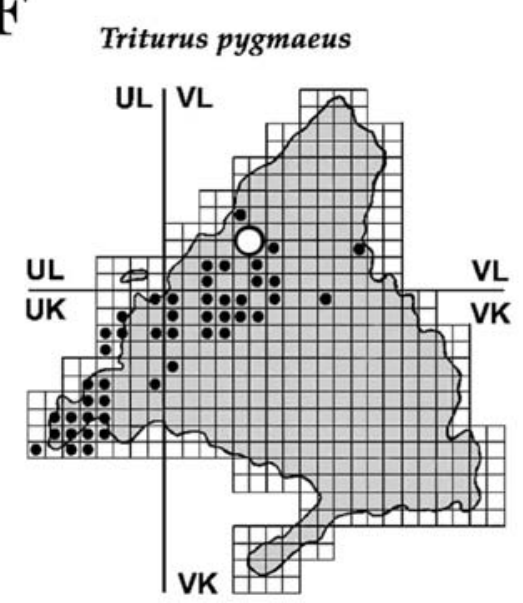


almanzoris Müller \& Hellmich, 1935, mientras que las demás corresponderían a la subespecie S. s. bejarae Wolterstorff, 1934, si bien es necesaria una revisión taxonómica de este último taxon (ver García-París et al., 2003; Martínez-Solano et al., 2005a). Por ello se ha preferido evaluar ambos taxones de manera conjunta en la propuesta de nuevas categorías de amenaza (ver más adelante).

\section{Pleurodeles waltl Michahelles 1830 (Fig. 1B)}

Presente en 69 cuadrículas UTM de 10x10 km (84 cuadrículas de $5 \times 5 \mathrm{~km}$ ). Se han encontrado poblaciones de esta especie entre 480 y 1540 metros de altitud. No se han podido confirmar los datos correspondientes a las cuadrículas: VL-00, VL-01, VL-11, VL-54, VK-26, VK-27, VK-37, VK-42, VK45, VK-47, VK-55, VK-57, VK-76, VK-79 y VK-87. Entre éstos, al menos los referentes a las poblaciones asentadas en las riberas del río Guadarrama (VK-26 y VK-27), la Laguna de Ontígola (VK-42) y el casco urbano de Madrid (VK-47) parecen constituir ausencias reales asociadas con la alteración o destrucción de medios acuáticos (Recuero-Gil et al., 2002; Martínez-Solano \& González-Fernández, 2003).

Los mapas de $5 \times 5 \mathrm{~km}$ muestran la existencia de una importante fragmentación en las poblaciones del centro y sur de la Comunidad de Madrid, que se encuentran aisladas en ocasiones decenas de kilómetros, especialmente en el sureste de la región.

\section{Mesotriton alpestris (Laurenti, 1768) (Fig. 1C)}

Presente en 2 cuadrículas UTM de 10x10 km (2 cuadrículas de $5 \times 5 \mathrm{~km}$ ). Las únicas poblaciones conocidas se asientan en el Parque Natural de Peñalara, entre 2080 y 2140 metros de altitud.

Los datos más recientes sugieren un estado de conservación aceptable para estas poblaciones, que parecen encontrarse en plena expansión (MartínezSolano et al., 2003). Por otro lado, nuevos estudios moleculares parecen confirmar el origen alóctono de estas poblaciones, si bien sigue sin determinarse el origen preciso de la introducción (Arano et al., 1991; M. Alcobendas \& M. García-París, com. pers. 2004).

\section{Lissotriton boscai (Lataste, 1879) (Fig. 1D)}

Presente en 19 cuadrículas UTM de 10x10 km (32 cuadrículas de $5 \times 5 \mathrm{~km}$ ). Se han encontrado poblaciones entre 500 y 1420 metros de altitud.

No se han confirmado las citas correspondientes a las cuadrículas VK-08, VK-19 y VK-29. Mientras que en el primer caso podría tratarse de un defecto de muestreo, las poblaciones de Collado-Villalba (VK-19) y Hoyo de Manzanares (VK-29) parecen haberse extinguido durante los últimos años (Recuero-Gil et al., 2002; Martínez-Solano \& González-Fernández, 2003), algo que en un futuro próximo podría suceder también en poblaciones próximas a éstas como en Alpedrete (MartínezSolano \& Bosch, 2001).

\section{Triturus marmoratus (Latreille, 1800) (Fig. 1E)}

Presente en 9 cuadrículas UTM de 10x10 km (13 cuadrículas de $5 \times 5 \mathrm{~km}$ ). Se han encontrado poblaciones entre 920 y 2140 metros de altitud.

No se ha podido confirmar la presencia de $T$. marmoratus en la cuadrícula VL-52, donde sí se ha registrado la extinción local de una población durante los últimos años (Martínez-Solano \& González-Fernández, 2003). Sin embargo, como indican estos mismos autores, probablemente en la zona existan otras poblaciones.

Aunque los mapas de distribución muestran que aparentemente las poblaciones de T. marmoratus se distribuyen en dos núcleos disjuntos, lo cierto es que a efectos prácticos en Madrid podemos considerar un único grupo localizado principalmente en la vertiente sur de la Sierra del Guadarrama, desde el Parque Natural de Peñalara a lo largo del valle del Lozoya y hasta la Sierra de La Cabrera, ya que el otro grupo lo constituyen poblaciones localizadas ya en territorio de la provincia de Segovia (San Rafael).

\section{Triturus pygmaeus (Wolterstorff, 1905) (Fig. 1F)}

Presente en 28 cuadrículas UTM de 10x10 km (43 cuadrículas de $5 \times 5 \mathrm{~km}$ ). Se han encontrado poblaciones entre 500 y 1420 metros de altitud, aunque excepcionalmente se han realizado observaciones esporádicas por encima de 2000 metros en

Fig. 1.- Mapas de distribución de los anfibios de la Comunidad de Madrid (I). Los círculos blancos indican citas que no han podido ser confirmadas recientemente. (A) Salamandra salamandra; (B) Pleurodeles waltl; (C) Mesotriton alpestris; (D) Lissotriton boscai; (E) Triturus marmoratus; (F) Triturus pygmaeus.

Fig. 1.- Distribution maps of amphibians in Comunidad de Madrid (I). White circles indicate records that were not confirmed recently. (A) Salamandra salamandra; (B) Pleurodeles waltl; (C) Mesotriton alpestris; (D) Lissotriton boscai; (E) Triturus marmoratus; (F) Triturus pygmaeus. 
el Parque Natural de Peñalara, atribuidas a ejemplares introducidos (ver Bosch et al., 2001).

De las citas bibliográficas, no se han podido confirmar las correspondientes a la cuadrícula VL21. En los mapas se han incluido tentativamente citas recientes procedentes de áreas alejadas del núcleo principal de la especie en Madrid, como en las cuadrículas VK-49 (Soto de Viñuelas) y VL-51 (Talamanca del Jarama). Estas citas podrían corresponder a $T$. marmoratus o a $T$. pygmaeus. Por lo demás, el mapa de $5 \times 5 \mathrm{~km}$ no muestra discontinuidades importantes en el área de distribución de esta especie en la Comunidad de Madrid.

\section{Alytes cisternasii Boscá, 1879 (Fig. 2A)}

Presente en 53 cuadrículas UTM de 10×10 km (65 cuadrículas de $5 \times 5 \mathrm{~km}$ ). Se han encontrado poblaciones entre 480 y 1300 metros de altitud. Siguiendo a Martínez-Solano \& García-París (2001), las citas correspondientes a las cuadrículas VK43, VK53 y VK73 no se consideran parte de la distribución de la especie en Madrid por corresponder posiblemente a errores de identificación.

No se han podido confirmar las citas de las cuadrículas UK-66, VK-05, VK-09, VK-19, VK-37, VK-47, VK-59, VL-44 y VL-61. Al menos en la localidad de Collado-Villalba (VK-19) se ha constatado la extinción local de una población por la destrucción de su lugar de reproducción (MartínezSolano \& González-Fernández, 2003), además de las citadas por Barbadillo \& García-París (1991). Los mismos autores señalan la posible desaparición de las poblaciones asentadas en los alrededores del área urbana de Madrid en los últimos años.

Los mapas de distribución a escala de $5 \times 5 \mathrm{~km}$ parecen mostrar cierto grado de continuidad entre las poblaciones localizadas, si bien aquellas que constituyen el límite sur del área de distribución de la especie en el centro de Madrid se encuentran más fragmentadas y presumiblemente aisladas unas de otras.

\section{Alytes obstetricans (Laurenti, 1768) (Fig. 2B)}

Presente en 31 cuadrículas UTM de 10x10 km (32 cuadrículas de $5 \times 5 \mathrm{~km}$ ). Se han encontrado poblaciones entre 490 y 2170 metros de altitud.

En este caso la mayor parte de las poblaciones cuya presencia actual no ha sido corroborada parecen haberse extinguido en el breve periodo de tiempo trascurrido entre la publicación del Atlas provisional de los anfibios y reptiles de Madrid (García-París et al., 1989) y los muestreos de los últimos años. Este parece ser el caso de las poblaciones localizadas en las cuadrículas UK-99, VK09, VK-53, VK-54, VK-67, VL-45, VL-54 y VL-55 (Martínez-Solano \& García-París, 2001; MartínezSolano \& González-Fernández, 2003).

Los mapas de distribución muestran la existencia de dos grupos de poblaciones bien delimitados geográficamente, que además corresponden desde un punto de vista genético, a diferentes linajes dentro de A. obstetricans (Martínez-Solano et al., 2004a). El primer grupo de poblaciones lo constituyen aquellas localizadas en las zonas más altas de la Sierra del Guadarrama, desde Peguerinos y El Escorial hasta Somosierra, asignadas provisionalmente a $A$. o. boscai Lataste, 1879 (MartínezSolano et al., 2004a). Actualmente, en lo que respecta a la vertiente madrileña de la Sierra, parecen existir poblaciones tan sólo en el Parque Natural de Peñalara, en el Circo del Nevero (Pinilla del Valle), así como en Cercedilla (Camorritos, $\mathrm{M}$. García-París, com. pers.). Al menos las dos primeras poblaciones se encuentran gravemente amenazadas de extinción a corto plazo debido a una epidemia de quitridiomicosis (Bosch et al., 2001; ver también www.sosanfibios.org). Por otro lado, las poblaciones del sureste madrileño corresponden a la subespecie $A$. o. pertinax García-París \& Martínez-Solano, 2001. Si bien parece existir cierta continuidad entre poblaciones, es patente también la existencia de núcleos aislados, como los localizados en las localidades de Villanueva de la Torre o Villasequilla de Yepes, en las provincias de

Fig. 2.- Mapas de distribución de los anfibios de la Comunidad de Madrid (II). Los círculos blancos indican citas que no han podido ser confirmadas recientemente. (A) Alytes cisternasii; (B) Alytes obstetricans; (C) Discoglossus galganoi / D. jeanneae; (D) Pelobates cultripes; (E) Pelodytes punctatus; (F) Bufo bufo. En 2C, los círculos grises y las "X" indican poblaciones asignadas respectivamente a $D$. galganoi y $D$. jeanneae mediante el uso de marcadores moleculares. Los asteriscos indican poblaciones en las que se encontraron haplotipos correspondientes a ambas especies.

Fig. 2.- Distribution maps of amphibians in Comunidad de Madrid (II). White circles indicate records that were not confirmed recently. (A) Alytes cisternasii; (B) Alytes obstetricans; (C) Discoglossus galganoi / D. jeanneae; (D) Pelobates cultripes; (E) Pelodytes punctatus; (F) Bufo bufo. In 2C, grey circles and " $\mathrm{X}$ " indicate populations assigned respectively to D. galganoi or to D. jeanneae by molecular markers. Asterisks indicate populations where haplotypes corresponding to both species were found. 
A

Alytes cisternasii

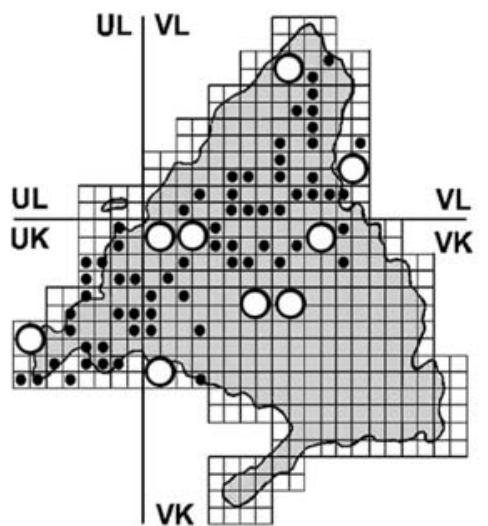

C Discoglossus galganoi Discoglossus jeanneae

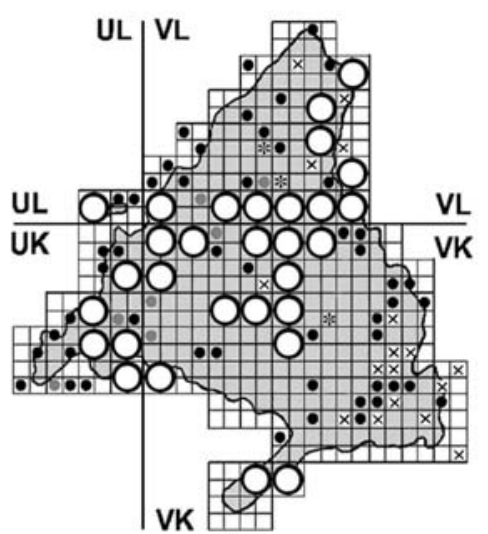

E Pelodytes punctatus

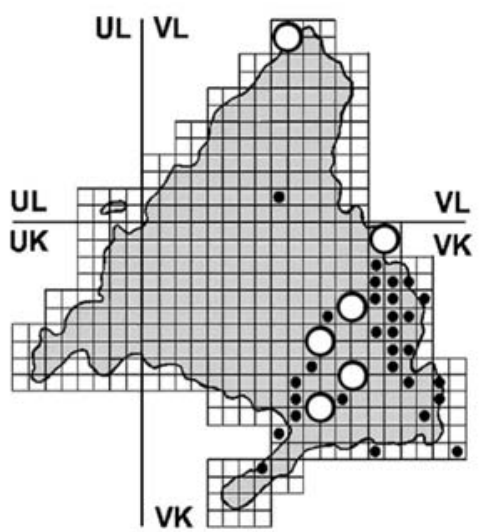

B

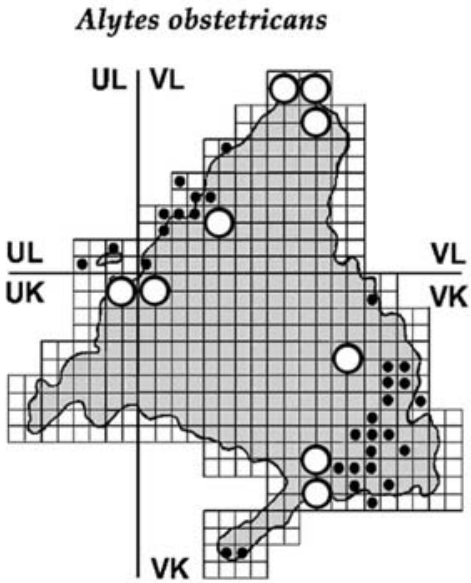

D

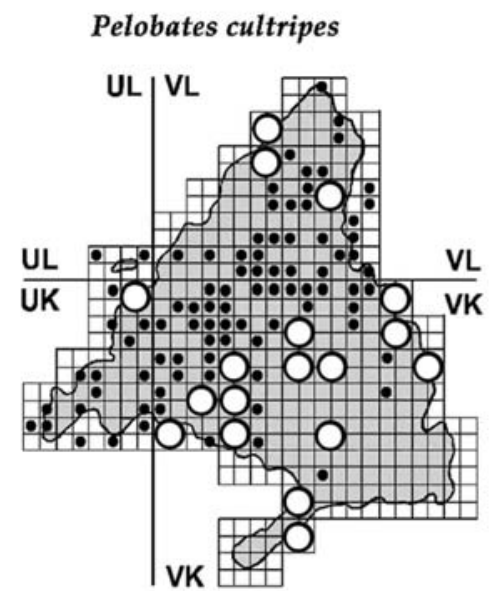

F

Bufo bufo

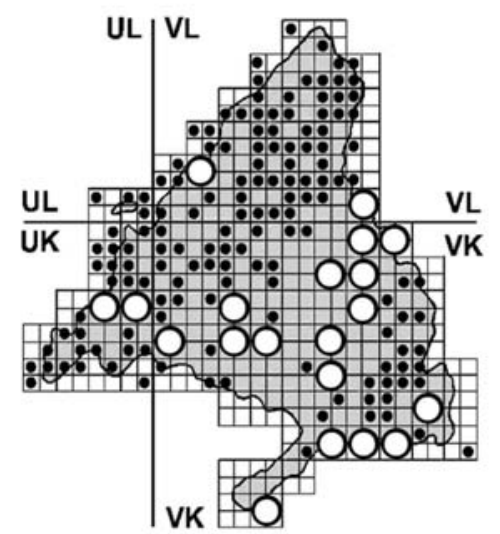


Guadalajara y Toledo, respectivamente. La extinción de poblaciones (como la de Loeches, ver Martínez-Solano \& García-París, 2001) está contribuyendo rápidamente a fragmentar las poblaciones de $A$. obstetricans en el sureste madrileño, con el consiguiente riesgo de extinción a medio plazo.

Discoglossus galganoi Capula, Nascetti, Lanza, Bullini y Crespo, 1985 / D. jeanneae Busack 1986 (Fig. 2C)

Presente en 87 cuadrículas UTM de 10x10 km (77 cuadrículas de $5 \times 5 \mathrm{~km}$ ). Se han encontrado poblaciones entre 480 y 1900 metros de altitud.

No se han confirmado las citas de las cuadrículas UL-80, UK-86, UK-87, UK-95, UK-96, UK-98, VL-00, VL-20, VL-30, VL-40, VL-50, VL-52, VL53, VL-60, VL-61, VL-64, VK-05, VK-08, VK-09, VK-19, VK-27, VK-32, VK-37, VK-39, VK-42, VK-46, VK-47, VK-48, VK-49, VK-59. En la mayor parte de los casos puede tratarse de defectos de prospección, si bien las poblaciones citadas en el área urbana de Madrid y sus alrededores podrían haberse extinguido durante los últimos años debido a la destrucción de sus puntos de reproducción (Martínez-Solano \& González-Fernández, 2003). En cualquier caso, resulta necesario llevar a cabo muestreos centrados de manera específica en esta especie para determinar con mayor rigor el grado de cohesión geográfica entre sus poblaciones.

En este trabajo se ha tratado la distribución de las dos especies de Discoglossus de manera conjunta, si bien en base a una combinación de análisis moleculares y modelos climáticos de la distribución de ambas especies (Martínez-Solano, 2004; Real et al., 2005; datos sin publicar) es posible delimitar de manera tentativa la distribución de las dos especies en la Comunidad de Madrid (Fig. 2C). Según estos resultados, D. galganoi se distribuye por el suroeste de Madrid, en la Sierra de la Higuera, a lo largo de los valles de los ríos Tiétar y Alberche y en zonas al pie de la Sierra del Guadarrama. Las citas de Peguerinos, San Rafael y El Espinar se han asignado de manera preliminar a $D$. galganoi, si bien es necesario llevar a cabo estudios moleculares para confirmar esta hipótesis. Discoglossus jeanneae ocupa las áreas calizas o yesíferas del este y sureste de la región así como zonas silíceas en las inmediaciones de Somosierra y Ayllón (Aoslos, Puebla de la Sierra, El Cardoso). Se han encontrado ejemplares con haplotipos correspondientes a ambas especies en simpatría en tres localidades: el Puerto de Canencia, Guadalix de la Sierra y RivasVaciamadrid. Otras posibles zonas de contacto incluirían las cuencas de los ríos Guadarrama y Jarama y el valle del Lozoya. Las poblaciones del área urbana de Madrid también se localizarían en zonas favorables para ambas especies.

\section{Pelobates cultripes (Cuvier, 1829) (Fig. 2D)}

Presente en 76 cuadrículas UTM de 10x10 km (88 cuadrículas de $5 \times 5 \mathrm{~km}$ ). Se han encontrado poblaciones entre 480 y 1470 metros de altitud.

Los registros de las cuadrículas UK-99, VL-33, VL-34, VL-52, VK-05, VK-16, VK-25, VK-26, VK-27, VK-42, VK-43, VK-47, VK-48, VK-55, VK-57, VK-78, VK-79, VK-87 no han podido confirmarse durante los muestreos de los últimos años. En algunas de estas cuadrículas se han extinguido poblaciones por desaparición de puntos de reproducción (VL-52, El Berrueco), en otras simplemente no existe confirmación reciente aunque probablemente haya ocurrido lo mismo en la mayoría (citas de la cuenca del río Guadarrama: VK-16, VK-25, VK-26, VK-27, ver Recuero-Gil et al., 2002; alrededores de Aranjuez y Laguna de Ontígola: VK-42, VK-43, M. Sánchez com. pers.; área urbana de Madrid y alrededores: VK-47, VK48, Martínez-Solano \& González-Fernández, 2003). En cualquier caso parece claro que las poblaciones localizadas al sur del núcleo principal del área de distribución de la especie en Madrid se encuentran prácticamente aisladas entre sí y por tanto seriamente amenazadas de extinción a medio plazo.

\section{Pelodytes punctatus (Daudin, 1802) (Fig. 2E)}

Presente en 28 cuadrículas UTM de 10x10 km (29 cuadrículas de $5 \times 5 \mathrm{~km}$ ). Se han encontrado poblaciones entre 490 y 880 metros de altitud.

El área de distribución de P. punctatus se limita fundamentalmente al sureste de la región. De hecho, existen únicamente dos citas relativamente alejadas de esta zona: la primera en los alrededores de San Agustín de Guadalix (Moncalvillo; G. Astudillo, com. pers.) y la segunda ya en la provincia de Segovia (Prádena; García-París et al., 1989, sin datos recientes).

Los núcleos de población más abundantes se asientan en el sureste de la región, siendo las citas más dispersas en sentido oeste.

\section{Bufo bufo (Linnaeus, 1758) (Fig. 2F)}

Presente en 97 cuadrículas UTM de 10x10 km (146 cuadrículas de $5 \times 5 \mathrm{~km}$ ). Se han encontrado poblaciones entre 470 y 2170 metros de altitud. 
No se han podido confirmar con citas recientes los datos acerca de la presencia de la especie en las cuadrículas UK-87, UK97, VK-06, VK-26, VK-27, VK-31, VK-36, VK-53, VK-55, VK-56, VK-58, VK-63, VK-67, VK-68, VK-69, VK-73, VK-79, VK-84, VL-11, VL-60. En algunos casos (VL-11 en Cercedilla, UK-87 y 97 en los alrededores del Pantano de San Juan, VL-60) la ausencia de citas refleja probablemente defectos de muestreo. Sin embargo en el caso de las citas del este y sureste de Madrid podría tratarse de ausencias reales, o al menos estar reflejando un posible declive de esta especie en la zona.

Las poblaciones de $B$. bufo se extienden por la mayor parte del territorio madrileño a excepción de una amplia franja que separa las poblaciones que ocupan el suroeste de la región y la Sierra del Guadarrama de las del sureste (Figura 2F). Entre ambos grupos de poblaciones tan sólo hay citas dispersas. Cabe señalar también la escasez de citas en los tramos inferiores del curso del río Guadarrama, no atribuibles a defectos de muestreo.

\section{Bufo calamita Laurenti, 1768 (Fig. 3A)}

Presente en 105 cuadrículas UTM de 10x10 km (216 cuadrículas de $5 \times 5 \mathrm{~km}$ ). Se han encontrado poblaciones entre 460 y 2200 metros de altitud.

De las citas históricas tan sólo quedan sin confirmar las cuadrículas VK-27, VK-37, VK-68 y VK-79. En la mayor parte de los casos se trata de poblaciones localizadas en las inmediaciones de grandes núcleos urbanos cuya supervivencia debe confirmarse con muestreos más específicos.

Bufo calamita mantiene poblaciones por la mayor parte del territorio madrileño, a excepción de las zonas más intensamente pobladas, aunque incluso en éstas presenta aún poblaciones marginales (por ejemplo en el Parque Juan Carlos I en Madrid). Las ausencias en las cuadrículas VL-63 y VL-64 parecen no obedecer a defectos de muestreo, sino más bien a la existencia de condiciones poco favorables para el asentamiento de poblaciones de esta especie en la zona.

Entre las poblaciones de $B$. calamita pueden establecerse dos grupos bien diferenciados desde un punto de vista biológico: las que se reproducen en medios acuáticos con altas concentraciones salinas y las que se reproducen en medios de agua dulce. En el sureste de Madrid existen poblaciones asentadas en medios temporales salobres, habitualmente considerados medios estresantes para las larvas de anfibios ya que disminuyen la supervivencia y alargan el periodo de desarrollo
(Gómez-Mestre \& Tejedo, 2003). Según han demostrado estudios de transplante recíproco, las poblaciones de medios salobres se encuentran especialmente adaptadas a las condiciones hiperosmóticas locales, de manera que siempre presentan valores de supervivencia y viabilidad superiores a los observados en poblaciones de medios dulces. En este sentido podrían ser consideradas como unidades de gestión independientes (sensu Crandall et al., 2000) y ser por tanto susceptibles de catalogación bajo alguna categoría de amenaza. Por el momento, se han localizado poblaciones con estas características en las localidades de Aranjuez, Colmenar de Oreja y RivasVaciamadrid.

\section{Hyla arborea (Linnaeus, 1758) (Fig. 3B)}

Presente en 38 cuadrículas UTM de 10x10 km (57 cuadrículas de $5 \times 5 \mathrm{~km}$ ). Se han encontrado poblaciones de $H$. arborea entre 620 y 2140 metros de altitud.

No se han podido confirmar las citas de las cuadrículas VL-11, VL-23, VL-34 y VL-52. En esta última cuadrícula se ha registrado la extinción local de una población por la desaparición de un punto de reproducción (Martínez-Solano \& González-Fernández, 2003), si bien como estos mismos autores señalan posiblemente existen otros núcleos de población de ésta y otras especies en los alrededores.

Las poblaciones de $H$. arborea se distribuyen fundamentalmente por las zonas de altitud media y alta de la Sierra del Guadarrama, desde El Escorial hasta Somosierra. Además existe otro núcleo de población en el suroeste de la región (Cenicientos, Cadalso de los Vidrios) que podría estar en contacto con el grupo de poblaciones de la Sierra en la zona de Valdemaqueda y Robledo de Chavela. Estas poblaciones se asientan generalmente en lugares de menor altitud y representan la continuación natural de las poblaciones del valle del Tiétar en la provincia de Avila.

\section{Hyla meridionalis Boettger, 1874 (Fig. 3C)}

Presente en 4 cuadrículas UTM de 10x10 km (8 cuadrículas de $5 \times 5 \mathrm{~km}$ ), con poblaciones entre los 480 y 830 metros de altitud.

La presencia de esta especie en Madrid ha sido confirmada recientemente (Aceituno, 2001). Ocupa zonas de media altitud en las inmediaciones de las Sierras de La Higuera y San Vicente y en los valles de los ríos Tiétar y Alberche, en muchos casos en simpatría estricta con $H$. arborea. 
A

Bufo calamita

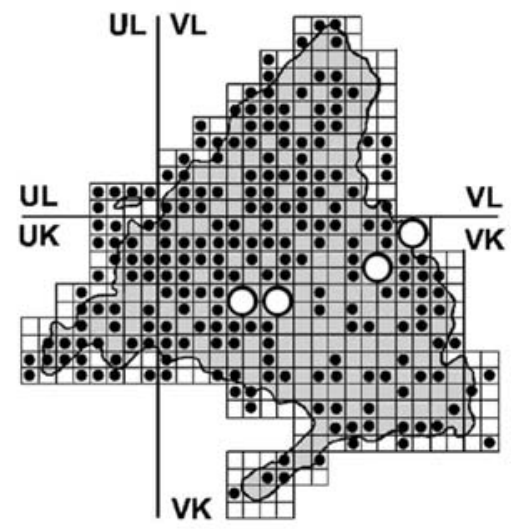

C

\section{Hyla meridionalis}

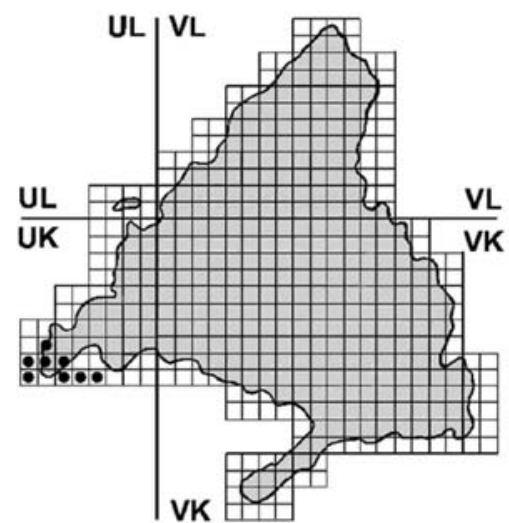

E

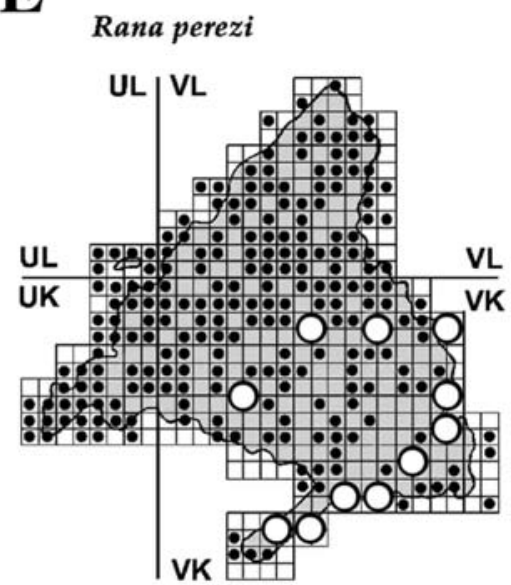

B

Hyla arborea

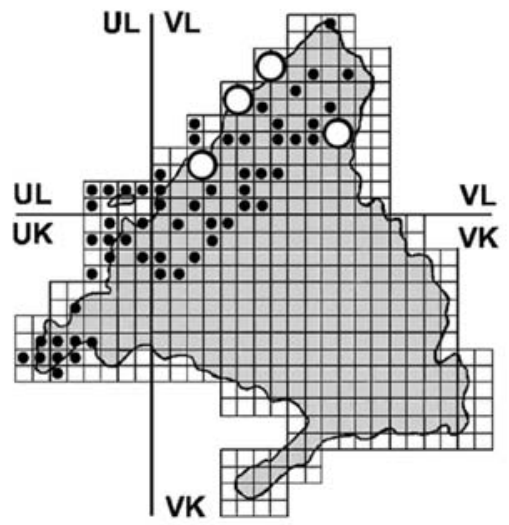

D

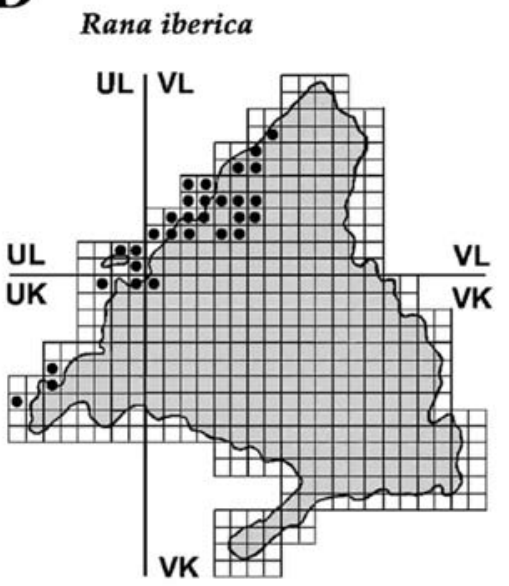

Fig. 3.- Mapas de distribución de los anfibios de la Comunidad de Madrid (III). Los círculos blancos indican citas que no han podido ser confirmadas recientemente. (A) Bufo calamita; (B) Hyla arborea; (C) Hyla meridionalis; (D) Rana iberica; (E) Rana perezi.

Fig. 3.- Distribution maps of amphibians in Comunidad de Madrid (III). White circles indicate records that were not confirmed recently. (A) Bufo calamita; (B) Hyla arborea; (C) Hyla meridionalis; (D) Rana iberica; (E) Rana perezi. 


\section{Rana iberica Boulenger, 1879 (Fig. 3D)}

Esta especie está presente en 17 cuadrículas UTM de 10x10 km (30 cuadrículas de 5x5 km). Se han encontrado poblaciones entre 660 y 2080 metros de altitud.

A una escala de análisis de $10 \times 10 \mathrm{~km}$ se ha confirmado la presencia de la especie en todas las cuadrículas donde había sido citada históricamente. Sin embargo, las poblaciones del entorno de El Escorial parecen haberse extinguido durante los últimos años (SCV, 2001a; Martínez-Solano \& González-Fernández, 2003).

Rana iberica se distribuye en dos núcleos principales en el área de estudio: uno en zonas medias y altas de la Sierra del Guadarrama, y otro en las estribaciones de la Sierra de Gredos en las localidades de El Tiemblo y La Adrada.

Se ha estudiado la variación genética de las poblaciones de $R$. iberica a lo largo del Sistema Central mediante el uso de marcadores moleculares nucleares (microsatélites) (Martínez-Solano et al., 2005b). Según estos resultados, las poblaciones del Sistema Central muestran una variabilidad genética muy reducida en comparación con otras poblaciones. Se ha propuesto que esto es una consecuencia de la combinación de factores históricos (colonización relativamente reciente del extremo oriental del Sistema Central a partir de un refugio glaciar localizado más al oeste) y otros más recientes (fragmentación creciente de las poblaciones por alteración y destrucción de hábitats, ver por ejemplo Bosch et al., 2006).

\section{Rana perezi Seoane, 1885 (Fig. 3E)}

Presente en 110 cuadrículas UTM de 10x10 km (221 cuadrículas de $5 \times 5 \mathrm{~km}$ ). Su rango de altitud oscila entre 460 y 2170 metros de altitud.

La mayor parte de las citas históricas han sido confirmadas. Entre aquellas que no han sido confirmadas recientemente se encuentran las siguientes cuadrículas: VK-26, VK-32, VK-42, VK-48, VK53, VK-63, VK-68, VK-74, VK-85, VK-86, VK-88.

Rana perezi se distribuye por la mayor parte del territorio madrileño, si bien las citas son más dispersas en el sureste de la región.

\section{Actualización de las categorías de Amenaza según criterios de la IUCN (2001)}

En la Tabla 1 se ofrecen los resultados del cálculo de los índices de continuidad y marginalidad para cada especie y la propuesta de catalogación en cada caso. Tan sólo en un caso (M. alpestris) existe una clara falta de continuidad entre las poblaciones madrileñas y las del resto del área de distribución de la especie en España. Sin embargo, dada la naturaleza alóctona de las poblaciones madrileñas (Arano et al., 1991; M. Alcobendas \& M. GarcíaParís, com. pers., 2004), se ha optado por recomendar mantener la categoría de amenaza vigente a nivel nacional.

Respecto al resto de los taxones, cuatro mostraron un nivel de continuidad que se consideró insuficiente para asegurar un posible "efecto rescate": A. o. pertinax, P. punctatus, $H$. meridionalis y $R$. iberica. Otros siete taxones presentan valores de continuidad intermedios: $S$. salamandra, L. boscai, T. marmoratus, T. pygmaeus, A. cisternasii, A. o. boscai y $D$. jeanneae. El resto (siete taxones) presenta valores de continuidad que justifican la reducción de las categorías de amenaza.

Según esta nueva propuesta, dos taxones se consideran "En Peligro": A. o. pertinax y R. iberica; cuatro "Vulnerable": S. salamandra, M. alpestris, T. pygmaeus y H. meridionalis; cuatro como "Casi Amenazada": A. cisternasii, A. o. boscai, D. jeanneae y $P$. punctatus. El resto (nueve especies) quedaría catalogado como "Preocupación Menor".

Los valores de marginalidad oscilan entre 0,018 (M. alpestris) y 0,973 (R. perezi). Las especies más ampliamente distribuidas en la Comunidad de Madrid de acuerdo con este índice son: $P$. waltl, $P$. cultripes, B. bufo, B. calamita y $R$. perezi.

En cuanto al establecimiento de prioridades de conservación, se ha propuesto la máxima para los siguientes taxones:

- Salamandra salamandra: Propuesta de catalogación: "Vulnerable". Justificación: en regresión en toda su área de distribución en Madrid (De la Riva et al., 1998; Martínez-Solano et al., 2003). Las poblaciones del Parque Natural de Peñalara parecen estar siendo afectadas ahora por la epidemia de quitridiomicosis que mantiene al borde de la extinción a la población de $A$. o. boscai de la zona (Bosch \& Martínez-Solano, 2006). Por otro lado, la distribución global de $S$. s. almanzoris es muy restringida (Martínez-Solano et al., 2005a) y la responsabilidad de la Comunidad de Madrid en la conservación de este taxon es muy elevada ya que la mayor parte de sus poblaciones se encuentran repartidas por su territorio.

- Alytes o. boscai: Propuesta de catalogación: "Casi Amenazada". Justificación: como se ha comentado anteriormente, sus poblaciones están sufriendo un fuerte declive por quitridiomicosis que podría afec- 


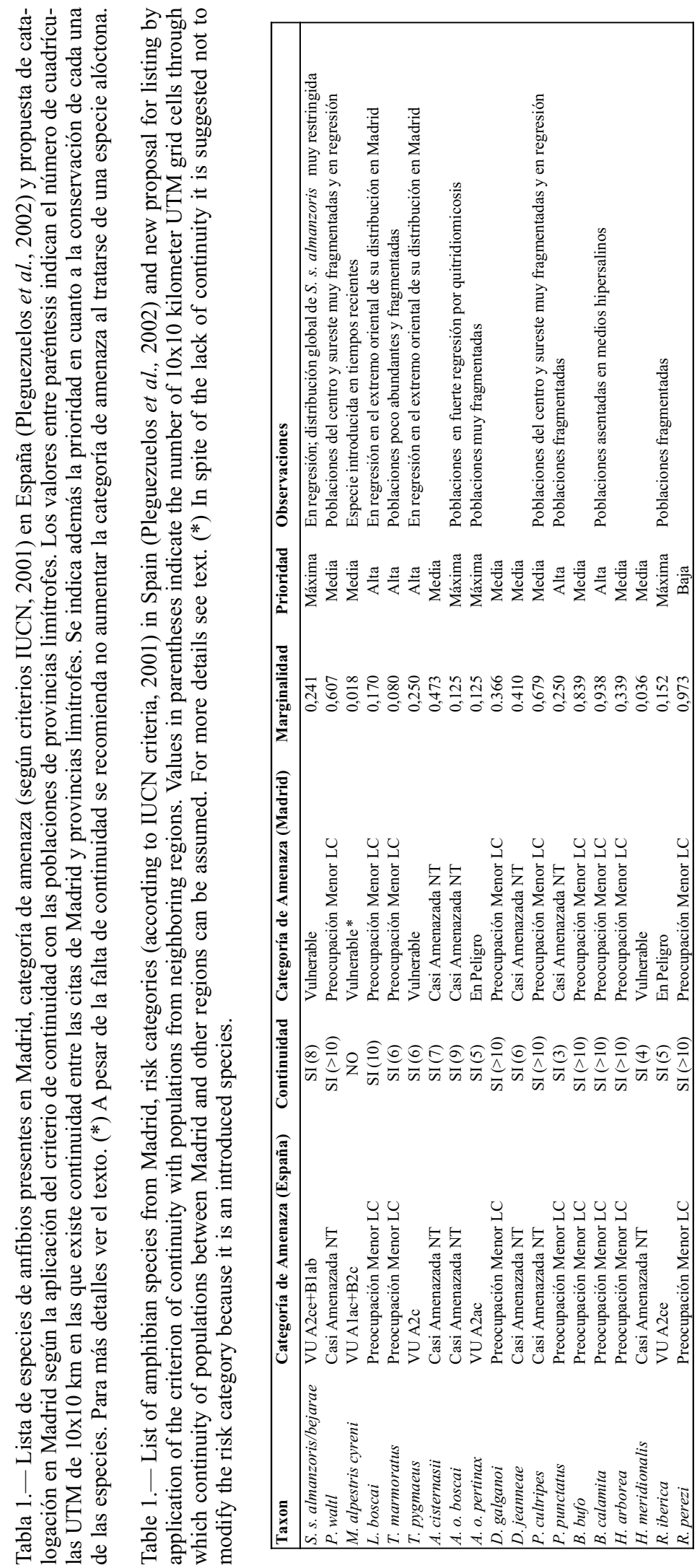

tar a toda su área de distribución en Madrid (Bosch et al., 2001; MartínezSolano et al., 2003; Martínez-Solano \& González-Fernández, 2003) y por extensión, podría acabar con las de provincias limítrofes, cuyo estatus debe ser reevaluado con urgencia.

- Alytes o. pertinax: Propuesta de catalogación: "En Peligro". Justificación: presenta poblaciones muy fragmentadas y asentadas en medios muy alterados, casi sin hábitats naturales de reproducción (ver García-París et al., 2000; Martínez-Solano et al., 2004b), por lo que su supervivencia a medio plazo está seriamente comprometida.

- Rana iberica: Propuesta de catalogación: "En Peligro". Justificación: poblaciones limitadas a áreas de montaña, en regresión en los últimos años y con una variabilidad genética reducida (SCV, 2001a; Esteban \& Martínez-Solano, 2002; MartínezSolano et al., 2005b; Bosch et al., 2006).

Para la mayor parte del resto de las especies se propone un grado de prioridad medio, en la mayor parte de los casos sobre la base de observaciones que sugieren una regresión continua de las poblaciones en los últimos años (Tabla 1).

\section{Discusión}

Los datos presentados en este trabajo pretenden servir de base para el estudio a largo plazo de la dinámica poblacional de las poblaciones de anfibios de la Comunidad de Madrid. Una primera cuestión a tener en cuenta es qué aportan los nuevos datos recogidos durante los últimos años. ¿Es posible inferir tendencias poblacionales comparando los nuevos datos con los de la década de los 80 (García-París et al., 1989)? Pese a lo limitado de las comparaciones posibles debido a diferencias en metodología, y como ya se ha comentado con anterioridad, puede pensarse que, en 
algunos casos, un esfuerzo de muestreo adicional podría aportar confirmación para algunas de las poblaciones citadas históricamente que no han sido localizadas en muestreos recientes. Sin embargo, en muchos casos los resultados de las prospecciones sugieren que la desaparición de poblaciones en los últimos años es un hecho. En particular, las citas históricas localizadas en el área urbana de Madrid y en sus alrededores representan probablemente la última constancia de una fauna residual que ha ido paulatinamente desapareciendo a medida que han ido creciendo los cada vez más numerosos núcleos urbanos de la periferia de la ciudad de Madrid, un fenómeno generalizado en la fauna asociada a ecosistemas fluviales en todo el mundo (ver Riley et al., 2005, y las referencias allí citadas).

Por otro lado, los estudios elaborados durante los últimos años han permitido delimitar con mayor precisión las unidades de conservación. A finales de los años 80 se tenía constancia de la existencia en la Comunidad de Madrid de 15 especies de anfibios, la mayoría comunes según los correspondientes mapas de distribución. Hoy la situación es muy distinta: al margen del caso de $H$. meridionalis, cuya existencia en la Comunidad de Madrid era considerada muy probable por GarcíaParís et al. (1989), la aplicación de marcadores moleculares (ADN mitocondrial, aloenzimas, microsatélites...) ha revelado la existencia de nuevos taxones en Madrid, como D. jeanneae, o bien ha permitido reevaluar el estatus taxonómico de otras, como T. pygmaeus. Además, otros estudios publicados durante la última década, con datos genéticos y ecológicos, han revelado diferencias significativas en la historia evolutiva de grupos de poblaciones dentro de algunas especies, como $S$. salamandra, $A$. obstetricans y $B$. calamita. Estos grupos de poblaciones presentan problemáticas específicas y deben ser considerados como unidades independientes a efectos de conservación, como se ha señalado en el apartado correspondiente a las categorías de amenaza.

Respecto a éstas, los nuevos datos sugieren la necesidad de introducir algunos cambios en la propuesta de De la Riva et al. (1998). Según la nueva propuesta, ninguna especie estaría catalogada como "En Peligro Crítico" (una en De la Riva et al., 1998), dos especies estarían incluidas en la categoría "En Peligro" (tres en De la Riva et al., 1998), cuatro como "Vulnerable" (cinco en De la Riva et al., 1998) y otras cuatro "Casi Amenazada" (cinco en De la Riva et al., 1998), lo que supone una reducción general en las categorí- as de amenaza. Por otro lado, ninguna especie queda incluida en la categoría "Datos Insuficientes", ya que tanto en los casos de D. galganoi / D. jeanneae como en el de P. punctatus se dispone ya de información suficiente para realizar una evaluación con garantías de su estado de conservación. En cualquier caso, es patente la necesidad de reevaluar periódicamente el estatus de conservación de las especies incluidas en los Catálogos Regionales de Especies Amenazadas, más aún considerando que, de seguir la tendencia actual de no adoptar medidas de protección en ningún caso, lo previsible es que el ya de por sí delicado estado de conservación de los anfibios madrileños no haga sino empeorar progresivamente.

Es ciertamente llamativo que más de la mitad de las especies de anfibios que habitan en la Comunidad de Madrid estén incluidas en alguna categoría de amenaza según los datos presentados y la metodología empleada en este trabajo. Entre los factores negativos identificados durante la elaboración de los muestreos se encuentra en primer lugar la pérdida continuada de hábitats terrestres y acuáticos y la consiguiente fragmentación de las poblaciones. En este sentido cabe destacar que la mayor parte de las áreas a través de las cuales puede asumirse cierto grado de continuidad entre las poblaciones madrileñas y las de provincias vecinas se encuentran en los alrededores de los valles de los ríos Tiétar y Alberche, o bien en los alrededores de los puertos de Guadarrama y Los Leones. En el sureste de la región la fragmentación es mucho mayor y, por tanto, la continuidad entre poblaciones que puede inferirse es mínima, aunque en este caso podría aducirse también una prospección insuficiente de las provincias limítrofes, algo que debe tenerse en cuenta en el futuro. Por tanto, la adecuada preservación de los corredores del oeste y suroeste así como la adopción de medidas para favorecer la continuidad de las poblaciones en el sureste (en particular, asegurando la buena calidad del agua de ríos y arroyos pero también creando y manteniendo adecuadamente nuevos puntos de reproducción) deben ser contemplados como medidas necesarias para la supervivencia a largo plazo de las poblaciones de anfibios.

Otras posibles medidas de conservación, complementarias con respecto a la adecuada conservación de los hábitats terrestres y acuáticos, incluirían aquellas que reducen el impacto de otros dos serios problemas detectados: la introducción de especies alóctonas y el atropello de ejemplares en carreteras. La gravedad de la generalización de introducciones 
de especies de crustáceos y peces alóctonos por parte de los pescadores e incluso por parte de anteriores Administraciones, y sus efectos negativos sobre las poblaciones autóctonas de anfibios han sido ampliamente reconocidos en la literatura científica (ver, por ejemplo, Aronsson \& Stenson, 1995; Axelsson et al., 1997; Beja \& Alcázar, 2003; Bosch et al., 2006; Drost \& Fellers, 1996; Fisher \& Shaffer, 1996; Gamradt \& Kats, 1996; Gamradt et al., 1997; Galán, 1997; Rodríguez et al., 2005). Como ha podido constatarse durante los muestreos, el problema de las introducciones incontroladas de especies de peces e invertebrados exóticos está muy extendido en toda la Comunidad de Madrid, si bien parece tener un efecto mayor en las zonas localizadas al pie de la Sierra del Guadarrama. Por el momento no se ha realizado un esfuerzo serio por parte de la Administración para atajar este problema, a excepción de medidas puntuales (Álvarez \& Martín, 2001). En zonas de alta montaña las repoblaciones inadecuadas con salmónidos en cabeceras de arroyos también han tenido consecuencias apreciables sobre las poblaciones autóctonas de anfibios, aunque en este caso sí se han adoptado medidas para paliar esta situación (como la eliminación de las poblaciones invasoras mediante pesca eléctrica en el Parque Natural de Peñalara).

En el caso de los atropellos, existe una cada vez más numerosa bibliografía acerca del impacto de esta causa de mortalidad en las poblaciones de muchas especies de anfibios en España (Lizana \& Barbadillo, 1997; López, 2001), e incluso se han identificado ya algunos "puntos negros" en la Comunidad de Madrid (SCV, 2001b). Durante nuestros muestreos se han observado también numerosos ejemplares atropellados en las carreteras y caminos de toda la Comunidad de Madrid. Por el momento, lamentablemente tampoco se han adoptado medidas para reducir el impacto de esta causa de mortalidad de manera generalizada, y pese al bajo coste de estas medidas correctoras, tan sólo existen barreras fijas para el paso de anfibios en un "punto negro" (entre las localidades de Torrelodones y Hoyo de Manzanares) en el Parque Regional de la Cuenca Alta del Manzanares.

Los datos presentados aquí deben constituir una base sólida sobre la cual fundamentar la gestión a largo plazo de las poblaciones de anfibios de Madrid. Esto no sólo implica adoptar las medidas necesarias en cada caso (como revisar las categorías de amenaza y proteger los medios de reproducción, o invertir en medidas correctoras) o profundizar en algunos aspectos aún insuficientemente conocidos de la biología e historia evolutiva de estos organismos (por ejemplo, aspectos demográficos básicos de la mayor parte de las especies), sino también mantener un seguimiento periódico, en especial sobre las especies más amenazadas. Los nuevos datos presentados muestran la importancia de actualizar la información biológica, genética y corológica para asegurar una gestión dinámica y por tanto más eficaz.

\section{AGRADECIMIENTOS}

Agradezco especialmente a Mario García-París y Borja Sanchiz su ayuda a lo largo del desarrollo del proyecto "Evaluación del conocimiento y del estado de conservación de la diversidad animal de Madrid" (GR/AMB/0750/2004), financiado por la Comunidad de Madrid. La Asociación Herpetológica Española facilitó el acceso a la base de datos de citas de anfibios de la Comunidad de Madrid. Agradezco al personal de los Parques Naturales de la "Cumbre, Circo y Lagunas de Peñalara» y del «Curso Medio del Río Guadarrama y su Entorno» su apoyo y colaboración. J. Aceituno, E. Albert, A. Álvarez, G. Astudillo, E. Ayllón, J. Barbadillo, J. Bocanegra, J. Bosch, D. Buckley, F. Cabezos, I. de la Riva, A. Doncel, P. Flores, C. Gábana, G. García, I. García-Paredes, J. García, L. García, M. García, M. García-París, M. Gómez, C. Grande, N. Guil, J. Hortal, S. Jiménez, M. Lapeña, A. López, J. I. López-Colón, B. Lorente, J. Manzanilla, F. Martínez, L. Martínez-Solano, P. Moreno, A. Murias, M. Nieto, M. París, M. Peinado, el grupo "Proyecto Verde" de Colmenarejo (gracias especialmente a Carlos, Roberto y Elvira), A. Quirós, E. Recuero, I. Rey, M. Salesa, I. Sánchez, M. Sánchez, I. Urbán y V. Quiralte aportaron citas desinteresadamente y/o colaboraron en los muestreos. Este estudio ha sido financiado por una beca predoctoral CAM-CSIC-MNCN.

\section{Referencias}

ACEITUNO, J., 2001. Hyla meridionalis (Ranita meridional), presencia en suroeste de Madrid y nuevas citas en Toledo. Boletín de la Asociación Herpetológica Española, 12: 9-10.

Alford, R. A. \& Richards, S. J., 1999. Global amphibian declines: a problem in applied ecology. Annual Review of Ecology and Systematics, 30: 133-165.

Álvarez, A. \& Martín, L., 2001. Apuesta por conservar los anfibios en un espacio protegido de Madrid. Quercus, 181: 6-7.

Álvarez-Vasserot, J. L., 1991. Extraña coloración de una larva de Alytes cisternasii. Boletín de la Asociación Herpetológica Española, 2: 19.

Arano, B., Arntzen, J. W., Herrero, P. \& GarciaPARís, M., 1991. Genetic differentiation among Iberian populations of the Alpine newt, Triturus alpestris. Amphibia-Reptilia, 12: 409-421.

Aronsson, S. \& Stenson, J. A. E., 1995. Newt-fish interactions in a small forest lake. Amphibia-Reptilia, 16: 177-184. 
Astudillo, G., García-París, M., Prieto, J. \& Rubio, J. L., 1993. Primeros datos sobre la distribución de anfibios y reptiles en la provincia de Guadalajara (Castilla-La Mancha, España). Revista Española de Herpetología, 7: 75-87.

Axelsson, E., Nyström, P., SidENMARK, J. \& BRÖNMARK, C., 1997. Crayfish predation on amphbian eggs and larvae. Amphibia-Reptilia, 18: 217-228.

Barbadillo, L. J. \& GARcíA-PARÍs, M., 1991. Problemas de conservación de los anfibios en España. Quercus, 62: 20-25.

BEJA, P. \& AlCÁzAR, R., 2003. Conservation of Mediterranean temporary ponds under agricultural intensification: an evaluation using amphibians. Biological Conservation, 114: 317-326.

Bosch, J., López, I., Cejudo, D. \& Álvarez, J. L., 1998. Parque Regional de la Cuenca Alta del Manzanares. In: X. Santos, M. A. Carretero, G. Llorente \& A. Montori (eds.). Inventario de las áreas importantes para los anfibios y reptiles de España. ICONA. Madrid: 1-237.

Bosch, J. \& Martínez-Solano, I., 2006. Chytrid fungus infection related to unusual mortalities of Salamandra salamandra and Bufo bufo in the Peñalara Natural Park (Central Spain). Oryx, 40: 84-89.

Bosch, J., Martínez-Solano, I. \& García-París, M., 2000. Inventario, estado de conservación y medidas correctoras para la conservación de los anfibios del Parque Natural de la Cumbre, Circo y Lagunas de Peñalara. Informe interno (inédito).

Bosch, J., Martínez-Solano, I. \& García-París, M., 2001. Evidence of a chytrid fungus infection involved in the decline of the common midwife toad (Alytes obstetricans) in protected areas of Central Spain. Biological Conservation, 97: 331-337.

Bosch, J., Rincón, P., Boyero, L. \& Martínez-Solano, I., 2006. Effects of introduced salmonids on a montane population of Iberian frogs. Conservation Biology, 20: 180-189.

Crandall, K. A., Bininda-Edmonds, O. R. P., Mace, G. M. \& WAYNE, R. K., 2000. Considering evolutionary processes in conservation biology. Trends in Ecology and Evolution, 15: 290-295.

Cushman, S. A., 2006. Effects of habitat loss and fragmentation on amphibians: a review and prospectus. Biological Conservation, 128: 231-240.

De la Riva, I., Dorda, J., García-París, M., LÓPez, P., Martín, J. \& SAlvador, A., 1998. Plan de acción de los anfibios y reptiles de la Comunidad de Madrid. Convenio de Colaboración CAM-CSIC (inédito).

Drost, C. A. \& Fellers, G. M., 1996. Collapse of a regional frog fauna in the Yosemite area of the California Sierra Nevada, USA. Conservation Biology, 10: 414-425.
Esteban, M. \& MARTíneZ-SolAno, I., 2002. Rana iberica. In: J. M. Pleguezuelos, R. Márquez \& M. Lizana (eds.). Atlas y Libro Rojo de los Anfibios y Reptiles de España. Dirección General de la Naturaleza Asociación Herpetológica Española ( $2^{\mathrm{a}}$ impresión), Madrid: 123-125.

Fisher, R. N. \& SHAFFER, H. B., 1996. The decline of amphibians in California's Great Central Valley. Conservation Biology, 10: 1387-1397.

Galán, P., 1997. Declive de poblaciones de anfibios en dos embalses de La Coruña (noroeste de España) por introducción de especies exóticas. Boletín de la Asociación Herpetológica Española, 8: 38-40.

GAMradt, S. C. \& Kats, L. B., 1996. Effect of introduced crayfish and mosquitofish on California newts. Conservation Biology, 10: 1155-1162.

Gamradt, S. C., Kats, L. B. \& Anzalone, C. B., 1997. Aggression by non-native crayfish deters breeding in California newts. Conservation Biology, 11: 793-796.

García-Avilés, J., Roblas Moreno, N. \& Hidalgo ZAMORA, J., 1999. Biodiversidad de los humedales del Parque Regional del Sureste. I. Vertebrados acuáticos. Informe interno (inédito).

GarcíA-PARÍs, M., 2002. Triturus pygmaeus. In: J. M. Pleguezuelos, R. Márquez \& M. Lizana (eds.). Atlas y Libro Rojo de los Anfibios y Reptiles de España. Dirección General de la Naturaleza - Asociación Herpetológica Española (2a impresión), Madrid: 70-72.

García-París, M., Alcobendas, M., Buckley, D. \& WAKE, D. B., 2003. Dispersal of viviparity across contact zones in Iberian populations of Fire Salamanders (Salamandra) inferred from discordance of genetic and morphological traits. Evolution, 57: 129-143.

García-París, M., Arano, B. \& Herrero, P., 2001. Molecular characterization of the contact zone between Triturus pygmaeus and T. marmoratus (Caudata: Salamandridae) in central Spain and their taxonomic assessment. Revista Española de Herpetología, 15: 115-126.

García-París, M., Astudillo, G., Prieto, J. \& MÁRQUEZ, R., 1990. Distribución de Alytes cisternasii Boscá, 1879, en el Centro de la Península Ibérica. Revista Española de Herpetología, 4: 87-92.

GARcíA-PArís, M. \& Jockusch, E. L., 1999. A mitochondrial DNA perspective on the evolution of Iberian Discoglossus. Journal of Zoology, London, 248: 209-218.

García-París, M., Martín, C., Dorda, J. \& Esteban, M., 1989. Atlas provisional de los anfibios y reptiles de Madrid. Revista Española de Herpetología, 3: 237-257.

García-París, M. \& Martínez-Solano, I., 2001. Nuevo estatus taxonómico para las poblaciones iberomediterráneas de Alytes obstetricans (Anura: Discoglossidae). Revista Española de Herpetología, 15: 99-113. 
GARCÍA-PARÍS，M., MARTíneZ-SOLANO, I. \& GARCÍA RomÁn, L., 2000. Situación crítica del sapo partero común en la Comunidad de Madrid. Quercus, 174: 50-51.

García-París, M., Montori, A. \& Herrero, P., 2004. Amphibia. Lissamphibia. En: Ramos, M. A. et al. (eds.). Fauna Iberica, vol. 24. Museo Nacional de Ciencias Naturales, CSIC. Madrid. 640 pp.

GARDENFÖRS, U., 2001. Classifying threatened species at national versus global levels. Trends in Ecology and Evolution, 16: 511-516.

Gardenförs, U., Hilton-Taylor, C., Mace, G. \& Rodríguez, J. P., 2001. The application of IUCN Red List Criteria at regional levels. Conservation Biology, 15: 1206-1212.

Gardenförs, U., Rodríguez, J. P., Hilton-Taylor, C., Hyslop, C., Mace, G., Molur, S. \& Poss, S., 2001. Draft guidelines for the application of IUCN Red List Criteria at national and regional levels. Species, 31-32: 58-70.

Gómez-Mestre, I. \& Tejedo, M., 2003. Local adaptation of an anuran amphibian to osmotically stressful environments. Evolution, 57: 1889-1899.

GRIJALBO, J., 1991. La laguna de San Juan y demás zonas húmedas del Tajuña. Agencia de Medio Ambiente de la Comunidad de Madrid. Madrid. 265 pp.

IUCN, 1994. IUCN Red List Categories. IUCN, Gland. www.redlist.org/info/categories criteria1994.

IUCN. 2001. IUCN Red List Categories: Version 3.1. IUCN. Gland \& Cambridge. 30 pp.

Keller, V., Zbinden, N., Schmid, H. \& Volet, B., 2005. A case study in applying the IUCN regional guidelines for national red lists and justifications for their modification. Conservation Biology, 19: 1827-1834.

Kiesecker, J. M., Blaustein, A. R. \& Belden, L. K., 2001. Complex causes of amphibian population declines. Nature, 410: 681-683.

Lizana, M., Arco, C., Morales, J. J., Bosch, J., Cejudo, J., López-GonzÁlez, F. J., Gutiérrez, J. \& MARTÍN-SÁNCHEZ, R., 1995. Atlas provisional de la herpetofauna en el Sistema Central segoviano. Revista Española de Herpetología, 9: 113-132.

Lizana, M. \& Barbadillo, L. J., 1997. Legislación, protección y estado de conservación de los anfibios y reptiles españoles. In: J. M. Pleguezuelos (ed.). Distribución y Biogeografía de los anfibios y reptiles en España y Portugal. Asociación Herpetológica Española - Universidad de Granada. Granada: 477516.

LÓPEZ, C., 2001. El impacto de las carreteras en las poblaciones de anfibios. Quercus, 183: 14-18.

MartínEZ-Solano, I., 2004. Phylogeography of Iberian Discoglossus (Anura: Discoglossidae). Journal of Zoological Systematics and Evoutionary Research, 42: 298-305.
MartíneZ-Solano, I., Alcobendas, M., Buckley, D. \& GARCÍA-PARÍs, M., 2005a. Molecular characterization of the endangered Salamandra salamandra almanzoris (Caudata, Salamandridae). Annales Zoologici Fennici, 42: 57-68.

Martínez-Solano, I. \& Bosch, J., 2001. Peligro para los anfibios de las canteras madrileñas de Alpedrete. Quercus, 188: 54-55.

Martínez-Solano, I., Bosch, J. \& García-París, M., 2003. Demographic trends and community stability in a montane amphibian assemblage. Conservation Biology, 17: 238-244.

Martínez-Solano, I. \& García-París, M., 2001. Distribución y estado de conservación de Alytes obstetricans y Pelodytes punctatus en el Sureste de Madrid. Boletín de la Asociación Herpetológica Española, 12: 37-41.

Martínez-Solano, I., García-París, M. \& JimÉnez, S., 2004b. Medidas para la conservación del sapo partero común en el sureste de Madrid. Quercus, 219: 3237.

Martínez-Solano, I., Gonçalves, H., Arntzen, J. W. \& GARcíA-PARís, M., 2004a. Phylogenetic relationships and biogeography of midwife toads (Discoglossidae, Alytes). Journal of Biogeography, 31: 603-618.

Martínez-Solano, I. \& GonZÁlEZ-FERnÁNDEZ, J. E., 2003. La colección de anfibios de Madrid del Museo Nacional de Ciencias Naturales y su utilidad en conservación. Graellsia, 59: 105-128.

Martínez-Solano, I., Rey, I. \& García-París, M., 2005b. The impact of historical and recent factors on genetic variability in a mountain frog: the case of Rana iberica (Anura: Ranidae). Animal Conservation, 8: 431-441.

Pleguezuelos, J. M., Márquez, R. \& Lizana, M., 2002. Atlas y Libro Rojo de los Anfibios y Reptiles de España. Dirección General de la Naturaleza Asociación Herpetológica Española ( $2^{\mathrm{a}}$ impresión), Madrid. 587 pp.

Pounds, J. A., Bustamante, M. R., Coloma, L. A., Consuegra, J. A., Fogden, M. P. L., Foster, P. N., La Marca, E., Masters, K. L., Merino-Viteri, A., Puschendorf, R., Ron, S. R., SANChez-Azofeifa, G. A., Still, C. J. \& Young, B. E., 2006. Widespread amphibian extinctions from epidemic disease driven by global warming. Nature, 439: 161-167.

Prieto, J., 1991. Nuevas cuadrículas para el atlas de anfibios y reptiles de Madrid. Boletín de la Asociación Herpetológica Española, 2: 20-21.

Real, R., Barbosa, A. M., Martínez-Solano, I. \& GARCÍA-PARÍs, M., 2005. Distinguishing the distributions of two cryptic Iberian frog species (Anura: Discoglossidae) on the basis of molecular data and environmental modeling. Canadian Journal of Zoology, 83: 536-545. 
Recuero-Gil, E., MartíneZ-Solano, I., Esteban, M. \& GARCíA-PARÍs, M., 2002. Inventario y estado de conservación de las poblaciones de anfibios del Parque Regional del Curso Medio del Río Guadarrama y su entorno. Informe interno (inédito).

Riley, S. P. D., Busteed, G. T., Kats, L. B., VAndergon, T. L., Lee, L. F. S., Dagit, R. G., Kerby, J. L., Fisher, R. N. \& SAUvaJot, R. M., 2005. Effects of urbanization on the distribution and abundance of amphibians and invasive species in southern California streams. Conservation Biology, 19: 1894-1907.

Rodríguez, C. F., Bécares, E., Fernández-AlÁez, M. \& FernándeZ-AlÁEZ, C., 2005. Loss of diversity and degradation of wetlands as a result of introducing exotic crayfish. Biological Invasions, 7: 75-85.
SCV. 2001a. Situación actual y problemas de conservación de Rana patilarga en la C.A.M. Boletín $S C V$, 89: 7-15.

SCV. 2001b. Atropello de anfibios en el valle del Lozoya. Quercus, 183: 19.

Stuart, S. N., Chanson, J. S., Cox, N. A., Young, B. E., Rodrigues, A. S. L., Fischman, D. L. \& Waller, R. W., 2004. Status and trends of amphibian declines and extinctions worldwide. Science, 306: 1783-1786. 
Apéndice.- - Listado de puntos de muestreo, localización (término municipal, cuadrículas UTM de 1x1 km y de 10x10 km, altitud) y especies observadas. Abreviaturas: $\mathrm{AV}=$ Ávila; $\mathrm{CU}=$ Cuenca; $\mathrm{GU}=$ Guadalajara; $\mathrm{SG}=\mathrm{Segovia}$; $\mathrm{TO}=\mathrm{Toledo}$.

Appendix.- List of sampling localities, localization (town, 1x1 and 10x10 kilometer UTM grids, altitude) and observed species. Abbreviations: $\mathrm{AV}=$ Ávila; $\mathrm{CU}=$ Cuenca; $\mathrm{GU}=$ Guadalajara; $\mathrm{SG}=$ Segovia; $\mathrm{TO}=$ Toledo.

\begin{tabular}{|c|c|c|c|c|}
\hline Municipio & UTM 1x1 & UTM 10x10 & Altitud & Especies presentes \\
\hline Ajalvir & VK-5787 & VK-58 & 720 & B. calamita; $R$. perezi \\
\hline Alameda del Valle & VL-2929 & VL-22 & 1100 & B. calamita ; R. perezi \\
\hline Alameda del Valle & VL-2830 & VL-23 & 1100 & R.perezi \\
\hline Alameda del Valle & VL-2831 & VL-23 & 1140 & B. calamita , R. perezi \\
\hline Alcalá de Henares & VK-6879/6978 & VK-67 & 600 & R. perezi; $B$. calamita \\
\hline Alcalá de Henares & VK-7079 & VK-77 & 620 & B. calamita \\
\hline Alcalá de Henares & VK-6482/6483 & VK-68 & 590 & \\
\hline Alcalá de Henares & VK-6486/6487 & VK-68 & 610 & A. cisternasii \\
\hline Alcobendas & VK-4387 & VK-48 & 680 & A. cisternasii \\
\hline Alcobendas & VK-4388 & VK-48 & 700 & \\
\hline Alcorcón & VK-2968 & VK-26 & 700 & B. calamita \\
\hline Aldea del Fresno & UK-9760 & UK-96 & 480 & B. bufo \\
\hline Aldea del Fresno & UK-9464/63 & UK-96 & 510 & \\
\hline Aldea del Fresno & UK-9766 & UK-96 & 500 & B. calamita \\
\hline Aldea del Fresno & UK-9765 & UK-96 & 480 & P. waltl \\
\hline Aldea del Fresno & UK-9765 & UK-96 & 480 & B. calamita \\
\hline Aldea del Fresno & UK-9765 & UK-96 & 480 & A. cisternasii \\
\hline Aldea del Fresno & VK-0063 & VK-06 & 490 & P. waltl; P. cultripes \\
\hline Aldea del Fresno & VK-0063 & VK-06 & 490 & B. calamita \\
\hline Aldea del Fresno & VK-0063 & VK-06 & 490 & B. calamita \\
\hline Aldea en Cabo (TO) & UK-7652 & UK-75 & 600 & A. cisternasii; H. meridionalis; $P$. waltl \\
\hline Aldealengua de Pedraza (SG) & VL-3138 & VL-33 & 1720 & B. bufo \\
\hline Aldealengua de Pedraza (SG) & VL-3247 & VL-34 & 1160 & T. marmoratus; Discoglossus; R. perezi \\
\hline Aldealengua de Pedraza (SG) & VL-3242 & VL-34 & 1420 & \\
\hline Almoguera (GU) & VK-9747 & VK-94 & 580 & \\
\hline Almorox (TO) & UK-8054 & UK-85 & 460 & B. calamita; $R$. perezi \\
\hline Almorox (TO) & UK-8362 & UK-86 & 760 & R. perezi \\
\hline Almorox (TO) & UK-8259 & UK-85 & 610 & R. perezi \\
\hline Almorox (TO) & UK-8053 & UK-85 & 480 & B. calamita \\
\hline Almorox (TO) & UK-8461 & UK-86 & 680 & B. bufo \\
\hline Almorox (TO) & UK-8356 & UK-85 & 590 & T. pygmaeus; $P$. waltl; A. cisternasii; R. perezi \\
\hline Almorox (TO) & UK-8263 & UK-86 & 760 & $\begin{array}{l}\text { B. calamita; A. cisternasii; } R \text {. perezi; } \text { T. pygmaeus; } H \text {. } \\
\text { arborea; } P \text {. waltl }\end{array}$ \\
\hline Almorox (TO) & UK-8364 & UK-86 & 770 & T. pygmaeus; L. boscai; R. perezi \\
\hline Almorox (TO) & UK-8260 & UK-86 & 640 & A. cisternasii; $R$. perezi \\
\hline Alovera (GU) & VK-7894 & VK-79 & 640 & R.perezi \\
\hline Alpedrete & VL-1302 & VL-10 & 800 & T. pygmaeus; R. perezi \\
\hline Alpedrete & VL-1400/1402 & VL-10 & 1000 & $\begin{array}{l}\text { R. perezi; } \text { T. pygmaeus; } P \text {. waltl; L. boscai; } B \text {. calamita; } H \text {. } \\
\text { arborea; A. cisternasii; } B \text {. bufo }\end{array}$ \\
\hline Ambite & VK-8564 & VK-86 & 640 & A. obstetricans; $B$. calamita \\
\hline Anchuelo & VK-7779 & VK-77 & 840 & B. calamita; $P$. punctatus \\
\hline Anchuelo & VK-7880 & VK-78 & 750 & B. bufo \\
\hline Anchuelo & VK-7378/7278 & VK-77 & 680 & B. bufo \\
\hline Anchuelo & VK-7278/7479/7580/7680 & VK-77/78 & 720 & B. bufo \\
\hline Añover de Tajo (TO) & VK -3625 & VK-32 & 460 & \\
\hline Añover de Tajo (TO) & VK-3526 & VK-32 & 490 & B. calamita; $P$. punctatus \\
\hline Aoslos & VL-4945 & VL-44 & 1070 & D. jeanneae; $R$. perezi \\
\hline
\end{tabular}




\begin{tabular}{|c|c|c|c|c|}
\hline Aranjuez & VK-3221 & VK-32 & 490 & B. calamita \\
\hline Aranjuez & VK-4930 & VK-43 & 570 & P. waltl; B. bufo \\
\hline Aranjuez & VK-4626 & VK-42 & 550 & B. calamita \\
\hline Aranjuez & VK-4738 & VK-43 & 500 & R. perezi \\
\hline Aranjuez & VK-2518 & VK-21 & 470 & R. perezi \\
\hline Aranjuez & VK-3621 & VK-32 & 510 & \\
\hline Aranjuez & VK-4224 & VK-42 & 530 & \\
\hline Aranjuez & VK-4225 & VK-42 & 530 & \\
\hline Aranjuez & VK-4425 & VK-42 & 540 & \\
\hline Arcones (SG) & VL-4050 & VL-45 & 1280 & R.perezi \\
\hline Arganda del Rey & VK-6557 & VK-65 & & P. waltl; R. perezi \\
\hline Arganda del Rey & VK-6263 & VK-66 & 580 & \\
\hline Arroyomolinos & VK-1957/2057/2158 & VK-15 & 560 & \\
\hline Arroyomolinos & VK-2157 & VK-25 & 610 & \\
\hline Arroyomolinos & VK-2059 & VK-25 & 600 & \\
\hline Azuqueca de Henares (GU) & VK-7888 & VK-78 & 600 & B. calamita; $R$. perezi \\
\hline Azuqueca de Henares (GU) & VK-7588 & VK-78 & 620 & R. perezi \\
\hline Barajas de Melo (CU) & VK-9542 & VK-94 & 600 & \\
\hline Barajas de Melo (CU) & VK-9642 & VK-94 & 590 & \\
\hline Batres & VK-2052 & VK-25 & 560 & \\
\hline Batres & VK-2052 & VK-25 & 560 & B. bufo; R. perezi \\
\hline Batres & VK-2152 & VK-25 & 630 & B. calamita \\
\hline Batres & VK-1954 & VK-15 & 550 & R. perezi \\
\hline Batres & VK-2155 & VK-25 & 640 & \\
\hline Batres & VK-1953 & VK-15 & 550 & R. perezi; B. calamita \\
\hline Batres & VK-1952 & VK-15 & 550 & B. bufo; A. cisternasii; R. perezi; B. calamita \\
\hline Batres & VK-1953 & VK-15 & 550 & R. perezi \\
\hline Batres & VK-2252 & VK-25 & 600 & B. bufo \\
\hline Becerril de la Sierra & VL-1708 & VL-10 & 1110 & B. bufo \\
\hline Becerril de la Sierra & VL-1707 & VL-10 & 1050 & B. calamita \\
\hline Becerril de la Sierra & VL-1709 & VL-10 & 1110 & $\begin{array}{l}\text { P. waltl; T. pygmaeus; B. calamita; H. arborea; P. cultripes; } \\
\text { A. cisternasii }\end{array}$ \\
\hline Becerril de la Sierra & VL-1808 & VL-10 & 1070 & B. calamita \\
\hline Becerril de la Sierra & VL-1807 & VL-10 & 1040 & $\begin{array}{l}\text { B. calamita; D. galganoi; T. pygmaeus; H. arborea; } P \text {. } \\
\text { cultripes }\end{array}$ \\
\hline Becerril de la Sierra & VL-1807 & VL-10 & 1010 & B. bufo; T. pygmaeus; A. cisternasii \\
\hline Becerril de la Sierra & VL-1907 & VL-10 & 1000 & $\begin{array}{l}\text { P. waltl; P. cultripes; } B \text {. bufo; B. calamita; } R \text {. perezi; } T \text {. } \\
\text { pygmaeus }\end{array}$ \\
\hline Belinchón (CU) & VK-9632 & VK-93 & 700 & \\
\hline Belinchón (CU) & VK-9633 & VK-93 & 700 & B. bufo; R. perezi; B. calamita; P. punctatus; D. jeanneae \\
\hline Belinchón (CU) & VK-9432 & VK-93 & 770 & \\
\hline Belinchón (CU) & VK-9636 & VK-93 & 640 & \\
\hline Belmonte de Tajo & VK-7145 & VK-74 & 720 & A. obstetricans \\
\hline Belmonte de Tajo & VK-7143 & VK-74 & 690 & A. obstetricans \\
\hline Belmonte de Tajo & VK-7141 & VK-74 & 760 & A. obstetricans \\
\hline Belmonte de Tajo & VK-7043 & VK-74 & 680 & A. obstetricans; D. jeanneae \\
\hline Berzosa de Lozoya & VL-5536/5535/5435 & VL-53 & 1020 & B. calamita; R. perezi \\
\hline Boadilla del Monte & VK- 2572 & VK-27 & 660 & R. perezi \\
\hline Boadilla del Monte & VK-2673 & VK-27 & 670 & \\
\hline Boadilla del Monte & VK-2474 & VK-27 & 670 & \\
\hline Braojos & VL-4543 & VL-44 & 1200 & \\
\hline Braojos & VL-4444 & VL-44 & 1240 & R. perezi \\
\hline Braojos & VL-4643 & VL-44 & 1180 & \\
\hline Braojos & VL-4346 & VL-44 & 1400 & S. salamandra \\
\hline Brea de Tajo & VK-9153 & VK-95 & 670 & \\
\hline Brea de Tajo & VK-9051 & VK-95 & 720 & P. punctatus; D. jeanneae \\
\hline Brunete & VK-1569 & VK-16 & 650 & B. calamita; $R$. perezi \\
\hline Brunete & VK-1569 & VK-16 & 650 & B. calamita \\
\hline Brunete & VK-1671 & VK-17 & 640 & \\
\hline Brunete & VK-1574 & VK-17 & 650 & P. waltl; P. cultripes; $B$. calamita \\
\hline Brunete & VK-1570 & VK-17 & 650 & B. calamita; R. perezi \\
\hline Brunete & VK-1572 & VK-17 & 640 & B. calamita \\
\hline Brunete & VK-1571 & VK-17 & 640 & B. calamita \\
\hline Brunete/Villaviciosa de Odón & VK-1968/1969 & VK-16 & 580 & R. perezi \\
\hline Bustarviejo & VL-4222 & VL-42 & 970 & R. perezi; P. waltl; H. arborea; T. marmoratus; B. bufo \\
\hline Bustarviejo & VL-3722 & VL-32 & 970 & P. waltl; R. perezi \\
\hline Bustarviejo & VL-3923 & VL-32 & 1270 & $P$. waltl; $P$. cultripes; $R$. perezi \\
\hline Bustarviejo & VL-4124 & VL-42 & 1230 & B. calamita; $R$. perezi \\
\hline Bustarviejo & VL-4023 & VL-42 & 1210 & B. bufo \\
\hline Bustarviejo & VL-4322 & VL-42 & 1110 & B. calamita \\
\hline Bustarviejo & VL-4221 & VL-42 & 1040 & B. bufo \\
\hline Bustarviejo & VL-4123 & VL-42 & 1140 & B. bufo \\
\hline Bustarviejo & VL-3520 & VL-32 & 1240 & S. salamandra \\
\hline Bustarviejo & VL-3524 & VL-32 & 1500 & B. calamita; S. salamandra \\
\hline Bustarviejo & VL-3622 & VL-32 & 1130 & B. bufo \\
\hline
\end{tabular}




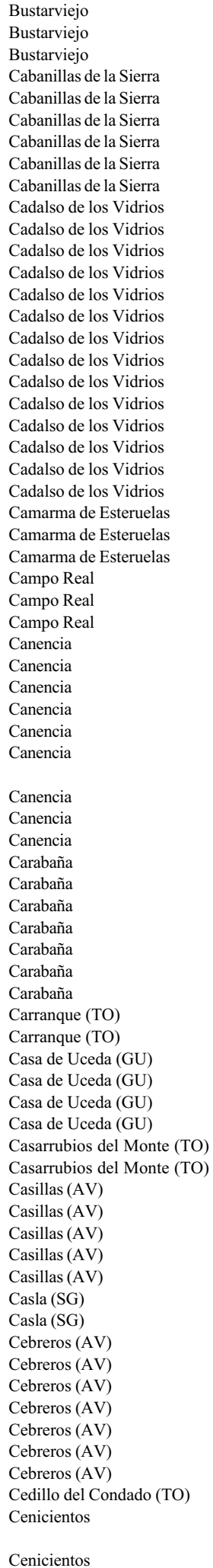

\begin{tabular}{c} 
VL-3521 \\
VL-3523/3524 \\
VL-4224 \\
VL-4719 \\
VL-4521 \\
VL-4720 \\
VL-4621 \\
VL-4521 \\
VL-4522 \\
UK-8064 \\
UK-7863 \\
UK-7860 \\
UK-7863 \\
UK-8164 \\
UK-7761 \\
UK-8161 \\
UK-7865 \\
UK-7863 \\
UK-8261 \\
UK-8061 \\
UK-8063 \\
UK-7963 \\
UK-8163 \\
VK-6889 \\
VK-6991 \\
VK-6988 \\
VK-6665 \\
VK-6765 \\
VK-6967 \\
VL-3729 \\
VL-3627 \\
VL-3830 \\
VL-3524 \\
VL-3425 \\
VL-3624/3625 \\
\\
\hline
\end{tabular}

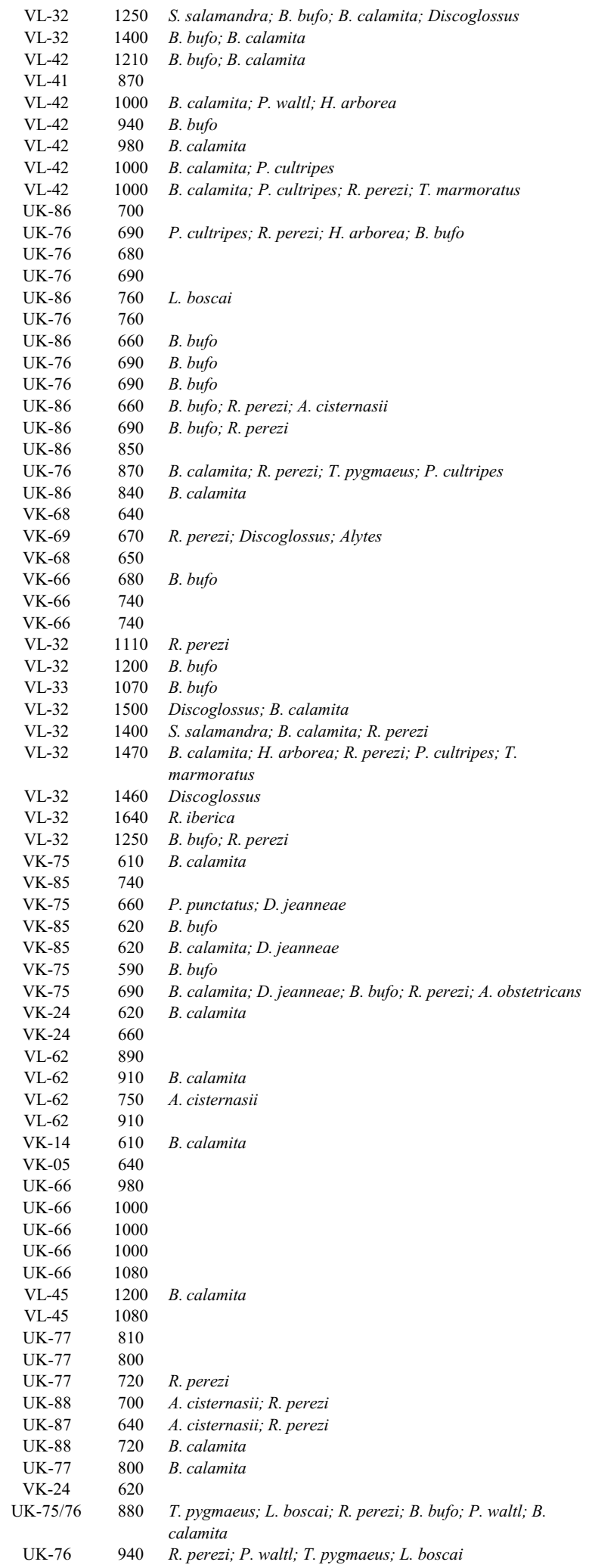




\begin{tabular}{|c|c|c|c|c|}
\hline Cenicientos & UK-7155/7156 & UK-75 & 800 & H. meridionalis \\
\hline Cenicientos & UK-7555 & UK-75 & 700 & B. calamita \\
\hline Cenicientos & UK-7556 & UK-75 & 720 & B. bufo; B. calamita \\
\hline Cenicientos & UK-7557 & UK-75 & 720 & B. bufo; B. calamita; H. arborea; R. perezi \\
\hline Cenicientos & UK-7655 & UK-75 & 670 & B. bufo \\
\hline Cenicientos & UK-6856 & UK-65 & 670 & R. perezi; L. boscai; T. pygmaeus \\
\hline Cenicientos & UK-7057 & UK-75 & 640 & $\begin{array}{l}\text { S. salamandra; A. cisternasii; B. calamita; } L . \text { boscai; } T \text {. } \\
\text { pygmaeus; } R \text {. perezi }\end{array}$ \\
\hline Cenicientos & UK-6757/6756 & UK-65 & 750 & B. bufo; $H$. arborea; $H$. meridionalis \\
\hline Cenicientos & UK-6957 & UK-65 & 710 & L. boscai; B. calamita \\
\hline Cenicientos & UK-7157 & UK-75 & 820 & A. cisternasii; B. calamita \\
\hline Cenicientos & UK-7054 & UK-75 & 800 & H. meridionalis \\
\hline Cenicientos & UK-7053 & UK-75 & 740 & D. galganoi; L. boscai \\
\hline Cenicientos & UK-6752 & UK-65 & 840 & \\
\hline Cenicientos & UK-7357 & UK-75 & 770 & H. meridionalis \\
\hline Cenicientos & UK-7256 & UK-75 & 830 & B. bufo; H. meridionalis \\
\hline Cenicientos & UK-7055 & UK-75 & 730 & B. bufo \\
\hline Cercedilla & VL-1114 & VL-11 & 1500 & S. salamandra \\
\hline Cercedilla & VL-0809 & VL-00 & 1160 & B. calamita; $P$. cultripes \\
\hline Cercedilla & VL-0915 & VL-01 & 1800 & R. iberica \\
\hline Cercedilla & VL-1014/1015 & VL-11 & 1600 & R. iberica; S. salamandra \\
\hline Cercedilla & VL-1214 & VL-11 & 1510 & R. iberica \\
\hline Cervera de Buitrago & VL-5630 & VL-53 & 900 & R. perezi \\
\hline Cervera de Buitrago & VL-5431/5531 & VL-53 & 950 & R. perezi \\
\hline Chapinería & UK-9669 & UK-96 & 660 & B. calamita \\
\hline Chapinería & UK-9771 & UK-97 & 670 & R. perezi; A. cisternasii; B. calamita; P. cultripes \\
\hline Chapinería & UK-9670 & UK-97 & 690 & R. perezi; $P$. cultripes \\
\hline Chapinería & UK-9570 & UK-97 & 690 & $\begin{array}{l}\text { R. perezi; B. calamita; D. galganoi; T. pygmaeus; L. boscai; } \\
\text { P. waltl; A. cisternasii }\end{array}$ \\
\hline Chapinería & UK-9670 & UK-97 & 680 & P. cultripes; B. calamita \\
\hline Chapinería & UK-9772 & UK-97 & 690 & B. calamita; $P$. cultripes; $P$. waltl \\
\hline Chapinería & UK-9673 & UK-97 & 710 & L. boscai; B. calamita; R. perezi \\
\hline Chinchón & VK-6145 & VK-64 & 640 & P. punctatus \\
\hline Chinchón & VK-6546 & VK-64 & 740 & \\
\hline Chinchón & VK-6342 & VK-64 & 740 & A. obstetricans; B. calamita \\
\hline Chinchón & VK-6545 & VK-64 & 720 & \\
\hline Chinchón & VK-6544 & VK-64 & 740 & D. jeanneae; $R$. perezi \\
\hline Chinchón & VK-5947 & VK-54 & 520 & B. calamita \\
\hline Chinchón & VK-6143 & VK-64 & 630 & \\
\hline Chinchón & VK-5745 & VK-54 & 510 & \\
\hline Chinchón & VK-6043 & VK-64 & 630 & D. jeanneae \\
\hline Chinchón & VK-5645 & VK-54 & 500 & B. bufo \\
\hline Ciempozuelos & VK-4742 & VK-44 & 530 & P. punctatus; $R$. perezi \\
\hline Ciempozuelos & VK-4947 & VK-44 & 520 & P. punctatus \\
\hline Ciempozuelos & VK-4644 & VK-44 & 580 & R.perezi \\
\hline Ciempozuelos & VK-5247 & VK-54 & 510 & R. perezi \\
\hline Ciempozuelos & VK-5144 & VK-54 & 540 & $\begin{array}{l}\text { B. bufo; B. calamita; D. jeanneae; P. cultripes; P. walt } ; R \text {. } \\
\text { perezi }\end{array}$ \\
\hline Ciempozuelos & VK-4641 & VK-44 & 530 & B. calamita \\
\hline Cobeña & VK-5891 & VK-59 & 700 & \\
\hline Cobeña & VK-5690 & VK-59 & 670 & B. calamita \\
\hline Collado Mediano & VL-1205 & VL-10 & 1080 & T. pygmaeus; $R$. perezi; B. calamita \\
\hline Colmenar de Oreja & VK-5534 & VK-53 & 500 & B. calamita \\
\hline Colmenar de Oreja & VK-6136 & VK-63 & 630 & B. calamita \\
\hline Colmenar de Oreja & VK-5035 & VK-53 & 540 & \\
\hline Colmenar de Oreja & VK-5036 & VK-53 & 540 & \\
\hline Colmenar de Oreja & VK-6540 & VK-64 & 740 & \\
\hline Colmenar de Oreja & VK-6838 & VK-63 & 740 & \\
\hline Colmenar de Oreja & VK-6739 & VK-63 & 740 & A. obstetricans \\
\hline Colmenar de Oreja & VK-6939 & VK-63 & 760 & A. obstetricans \\
\hline Colmenar de Oreja & VK-6639 & VK-63 & 730 & A. obstetricans \\
\hline Colmenar de Oreja & VK-5437 & VK-53 & 580 & B. calamita \\
\hline Colmenar de Oreja & VK-6238 & VK-63 & 650 & B. calamita \\
\hline Colmenar de Oreja & VK-6338 & VK-63 & 660 & B. calamita \\
\hline Colmenar de Oreja & VK-6138 & VK-63 & 630 & \\
\hline Colmenar de Oreja & VK-7238 & VK-73 & 660 & \\
\hline Colmenar de Oreja & VK-7436 & VK-73 & 590 & \\
\hline Colmenar de Oreja & VK-7338 & VK-73 & 660 & B. calamita \\
\hline Colmenar del Arroyo & VK-0071 & VK-07 & 570 & B. bufo; R. perezi; A. cisternasii \\
\hline Colmenar del Arroyo & UK-9775 & UK-97 & 710 & P. waltl \\
\hline Colmenar del Arroyo & UK-9773 & UK-97 & 710 & L. boscai \\
\hline Colmenar del Arroyo & UK-9774 & UK-97 & 710 & P. waltl; L. boscai; R. perezi \\
\hline Colmenar Viejo & VL-3808 & VL-30 & 1040 & R. perezi \\
\hline Colmenar Viejo & VL-4104 & VL-40 & 840 & A. cisternasii; R. perezi \\
\hline
\end{tabular}




\begin{tabular}{|c|c|c|c|c|}
\hline Colmenar Viejo & VL-3702 & VL-30 & 840 & R. perezi; A. cisternasii \\
\hline Colmenar Viejo & VL-3702 & VL-30 & 840 & P. waltl; B. calamita; $R$. perezi \\
\hline Colmenar Viejo & VL-4005 & VL-40 & 950 & B. calamita \\
\hline Colmenar Viejo & VL-4106 & VL-40 & 950 & B. calamita \\
\hline Colmenar Viejo & VL-4004 & VL-40 & 930 & B. calamita \\
\hline Colmenar Viejo & VL-3704 & VL-30 & 900 & P. cultripes \\
\hline Colmenar Viejo & VL-4203 & VL-40 & 850 & R. perezi \\
\hline Colmenar Viejo & VL-3704 & VL-30 & 880 & R.perezi; P. cultripes; B. bufo \\
\hline Colmenar Viejo & VK-3398 & VK-39 & 800 & P. waltl; T. pygmaeus; R. perezi; P. cultripes \\
\hline Colmenar Viejo & VK-3397 & VK-39 & 760 & R. perezi \\
\hline Colmenar Viejo & VL-3500 & VL-30 & 800 & \\
\hline Colmenar Viejo & VK-3499 & VK-39 & 820 & B. bufo \\
\hline Colmenar Viejo & VK-3498 & VK-39 & 780 & P. waltl; B. calamita \\
\hline Colmenar Viejo & VK-5099 & VK-59 & 600 & \\
\hline Colmenar Viejo & VL-3809 & VL-30 & 1040 & \\
\hline Colmenar Viejo & VL-3305 & VL-30 & 930 & B. calamita; P. cultripes; $R$. perezi \\
\hline Colmenar Viejo & VK-4399 & VK-49 & 710 & B. calamita \\
\hline Colmenar Viejo & VK-4399 & VK-49 & 710 & B. calamita \\
\hline Colmenar Viejo & VL-4300 & VL-40 & 720 & B. bufo \\
\hline Colmenar Viejo & VL-4300 & VL-40 & 720 & B. calamita \\
\hline Colmenar Viejo & VL-4300 & VL-40 & 720 & B. calamita \\
\hline Colmenar Viejo & VL-4300 & VL-40 & 720 & B. calamita \\
\hline Colmenar Viejo & VL-4300 & VL-40 & 720 & B. calamita \\
\hline Colmenar Viejo & VL-4301 & VL-40 & 720 & B. bufo; B. calamita \\
\hline Colmenar Viejo & VL-4301 & VL-40 & 720 & R. perezi; B. bufo \\
\hline Colmenarejo & VK-1483 & VK-18 & 750 & \\
\hline Colmenarejo & VK-1588 & VK-18 & 860 & \\
\hline Colmenarejo & VK-1385 & VK-18 & 730 & R. perezi \\
\hline Colmenarejo & VK-1583 & VK-18 & 660 & R.perezi \\
\hline Colmenarejo & VK-1488 & VK-18 & 840 & $\begin{array}{l}\text { T. pygmaeus; R. perezi; P. cultripes; P. waltl; B. bufo; } B \text {. } \\
\text { calamita }\end{array}$ \\
\hline Colmenarejo & VK-1488 & VK-18 & 830 & B. calamita; T. pygmaeus; P. cultripes; R. perezi \\
\hline Colmenarejo & VK-1588 & VK-18 & 830 & T. pygmaeus; $P$. waltl; R. perezi \\
\hline Colmenarejo & VK-1588 & VK-18 & 830 & P. cultripes; B. calamita; R. perezi \\
\hline Colmenarejo & VK-1288 & VK-18 & 830 & R. perezi \\
\hline Colmenarejo & VK-1288 & VK-18 & 830 & T. pygmaeus; P. waltl; B. calamita; R. perezi \\
\hline Colmenarejo & VK-1288 & VK-18 & 830 & R. perezi; P. waltl \\
\hline Colmenarejo & VK-1391 & VK-18 & 870 & R.perezi \\
\hline Colmenarejo & VK-1391 & VK-18 & 870 & P. waltl; B. calamita \\
\hline Colmenarejo & VK-1391 & VK-18 & 870 & P. cultripes; R. perezi; B. calamita; $P$. waltl; T. pygmaeus \\
\hline Colmenarejo & VK-1288 & VK-18 & 800 & B. bufo \\
\hline Colmenarejo & VK-1188 & VK-18 & 800 & B. bufo \\
\hline Colmenarejo & VK-1193/1293 & VK-19 & 830 & \\
\hline Corpa & VK-8077 & VK-87 & 840 & B. calamita \\
\hline Corpa & VK-7776 & VK-77 & 830 & B. calamita; $P$. punctatus; $D$. jeanneae \\
\hline Corpa & VK-7774 & VK-77 & 810 & D. jeanneae \\
\hline Corpa & VK-7975 & VK-77 & 820 & B. calamita; $P$. punctatus; D. jeanneae \\
\hline Corpa & VK-7772 & VK-77 & 820 & A. obstetricans; D. jeanneae; P. punctatus; B. calamita \\
\hline Daganzo de Arriba & VK-6189/6194 & VK-68 & 680 & P. cultripes \\
\hline Daganzo de Arriba & VK-6092 & VK-69 & 720 & R. perezi \\
\hline Daganzo de Arriba & VK-6193 & VK-69 & 710 & R. perezi \\
\hline Daganzo de Arriba & VK-6491 & VK-69 & 670 & \\
\hline Driebes (GU) & VK-9752 & VK-95 & 620 & B. calamita \\
\hline Driebes (GU) & VK-9655 & VK-95 & 720 & D. jeanneae \\
\hline Driebes (GU) & VK-9749 & VK-94 & 570 & \\
\hline Driebes (GU) & VK-9750 & VK-95 & 570 & R. perezi \\
\hline El Álamo & VK-1654 & VK-15 & 580 & P. cultripes \\
\hline El Álamo & VK-1753 & VK-15 & 580 & B. bufo \\
\hline El Álamo & VK-1754 & VK-15 & 580 & B. calamita \\
\hline El Atazar & VL-6031 & VL-63 & 910 & B. bufo \\
\hline El Atazar & VL-6032 & VL-63 & 910 & R. perezi \\
\hline El Berrueco & VL-5125 & VL-52 & 1020 & B. bufo \\
\hline El Berrueco & VL-5226 & VL-52 & 930 & \\
\hline El Berrueco & VL-5325 & VL-52 & 960 & P. waltl; B. calamita R. perezi \\
\hline El Berrueco & VL-5326 & VL-52 & 960 & R. perezi \\
\hline El Berrueco & VL-5227 & VL-52 & 950 & \\
\hline El Berrueco & VL-5424 & VL-52 & 1060 & B. bufo \\
\hline El Cardoso de la Sierra (GU) & VL-6448 & VL-64 & 1250 & \\
\hline El Cardoso de la Sierra (GU) & VL-6549 & VL-64 & 1340 & \\
\hline El Cardoso de la Sierra (GU) & VL-6248 & VL-64 & 1260 & R. perezi \\
\hline El Cardoso de la Sierra (GU) & VL-6349 & VL-64 & 1230 & B. bufo \\
\hline El Casar (GU) & VL-6504 & VL-60 & 830 & B. calamita \\
\hline El Casar (GU) & VL-6307 & VL-60 & 820 & R. perezi \\
\hline El Casar (GU) & VL-6307 & VL-60 & 840 & \\
\hline El Casar (GU) & VL-6512 & VL-61 & 790 & \\
\hline
\end{tabular}




\begin{tabular}{|c|c|}
\hline El Casar (GU) & \\
\hline El Cubillo de Uceda (GU & \\
\hline El Cubillo de Uceda (GU & \\
\hline El Cubillo de Uceda (GU & \\
\hline El Cubillo de Uceda (GU & \\
\hline El Cubillo de Uceda (GU & \\
\hline ElEscorial & \\
\hline ElEscorial & \\
\hline ElEscorial & \\
\hline ElEscorial & \\
\hline ElEscorial & \\
\hline ElEscorial & \\
\hline El Espinar (SG) & \\
\hline El Espinar (SG) & \\
\hline El Espinar (SG) & \\
\hline El Espinar (SG) & \\
\hline El Espinar (SG) & \\
\hline El Espinar (SG) & \\
\hline El Espinar (SG) & \\
\hline El Espinar (SG) & \\
\hline El Espinar (SG) & \\
\hline El Espinar (SG) & \\
\hline El Espinar (SG) & \\
\hline El Espinar (SG) & \\
\hline El Espinar (SG) & \\
\hline El Espinar (SG) & \\
\hline El Espinar (SG) & \\
\hline El Espinar (SG) & \\
\hline El Espinar (SG) & \\
\hline El Espinar (SG) & \\
\hline El Espinar (SG) & \\
\hline El Espinar (SG) & \\
\hline El Espinar (SG) & \\
\hline El Espinar (SG) & \\
\hline El Espinar (SG) & \\
\hline El Espinar (SG) & \\
\hline El Espinar (SG) & \\
\hline El Espinar (SG) & \\
\hline El Espinar (SG) & \\
\hline El Espinar (SG) & \\
\hline El Espinar (SG) & \\
\hline El Espinar (SG) & \\
\hline El Espinar (SG) & \\
\hline El Espinar (SG) & \\
\hline El Espinar (SG) & \\
\hline El Hoyo de Pinares (AV) & \\
\hline El Hoyo de Pinares (AV) & \\
\hline El Hoyo de Pinares (AV) & \\
\hline El Hoyo de Pinares (AV) & \\
\hline El Hoyo de Pinares (AV) & \\
\hline El Hoyo de Pinares (AV) & \\
\hline El Hoyo de Pinares (AV) & \\
\hline El Hoyo de Pinares (AV) & \\
\hline El Hoyo de Pinares (AV) & \\
\hline El Hoyo de Pinares (AV) & \\
\hline El Hoyo de Pinares (AV) & \\
\hline El Hoyo de Pinares (AV) & \\
\hline El Molar & \\
\hline E1 Molar & \\
\hline El Molar & \\
\hline El Molar & \\
\hline El Molar & \\
\hline El Molar & \\
\hline El Molar & \\
\hline El Tiemblo (AV) & \\
\hline El Tiemblo (AV) & \\
\hline El Tiemblo (AV) & \\
\hline
\end{tabular}

\begin{tabular}{|c|c|c|c|}
\hline VL-6511 & VL-61 & 860 & B. calamita \\
\hline VL-6519 & VL-61 & 880 & R. perezi; B. calamita \\
\hline VL-6620 & VL-62 & 910 & P. cultripes \\
\hline VL-6720 & VL-62 & 910 & \\
\hline VL-6216 & VL-61 & 860 & P. waltl; Bufo; P. cultripes; R. perezi \\
\hline VL-6917 & VL-61 & 910 & R. perezi \\
\hline VK-1194 & VK-19 & 840 & R. perezi \\
\hline VK-1093/1193 & VK-19 & 830 & P. waltl; P. cultripes; $R$. perezi \\
\hline VK-0993 & VK-09 & 880 & P. cultripes; P. waltl; R. perezi; B. bufo; B. calamita \\
\hline VK-0388 & VK-08 & 970 & $\begin{array}{l}\text { H. arborea; B. calamita; R. perezi; T. pygmaeus; } P \text {. waltl; B. } \\
\text { bufo }\end{array}$ \\
\hline VK-0588 & VK-08 & 910 & B. calamita \\
\hline VK-0388 & VK-08 & 970 & P. waltl \\
\hline UL-9406 & UL-90 & 1300 & S. salamandra \\
\hline UL-9508 & UL-90 & 1200 & H. arborea; $R$. perezi \\
\hline UL-8807/8808 & UL-80 & 1240 & H. arborea; $R$. perezi \\
\hline UL-9108 & UL-90 & 1150 & R. perezi \\
\hline VL-0815 & VL-01 & 1640 & S. salamandra \\
\hline UL-9305/9306 & UL-90 & 1350 & Discoglossus; B. calamita \\
\hline VL-0615 & VL-01 & 1550 & R. iberica \\
\hline UL-9205 & UL-90 & 1550 & S. salamandra \\
\hline UL-8206/8306 & UL-80 & 1250 & P. cultripes; B. calamita; H. arborea; $R$. perezi \\
\hline UL-8908/9008 & UL- $80 / 90$ & 1250 & B. calamita; H. arborea; $R$. perezi \\
\hline UL-9408 & UL-90 & 1200 & $\begin{array}{l}\text { Discoglossus; } \text { A. obstetricans; B. calamita; } R \text {. iberica; } P . \\
\text { walt } ; \text { B. bufo; H. arborea }\end{array}$ \\
\hline UL-8507 & UL-80 & 1300 & R. perezi \\
\hline VL-0716/0714 & VL-01 & 1580 & $\begin{array}{l}\text { R. iberica; B. bufo; S. salamandra; R. perezi; A. obstetricans; } \\
\text { B. calamita }\end{array}$ \\
\hline VL-0010 & VL-01 & 1200 & B. bufo; B. calamita \\
\hline UL-8305 & UL- 80 & 1250 & P. cultripes: $B$. bufo: $B$. calamita $: R$. perezi \\
\hline UL-8304 & UL-80 & 1250 & $\begin{array}{l}\text { T. marmoratus; A. obstetricans; B. calamita; } H . \text { arborea; } R \text {. } \\
\text { perezi }\end{array}$ \\
\hline UL-8406 & UL- 80 & 1250 & P. cultripes: $B$. bufo $: H$ arborea $: R$. perezi $B$. calamita \\
\hline UL-8905 & UL- 80 & 1400 & R. perezi \\
\hline UL-9706 & UL-90 & 1350 & S. salamandra \\
\hline VL-0614 & VL-01 & 1390 & A. obstetricans; B. bufo; B. calamita; R. iberica; R. perezi \\
\hline VL-0312 & VL-01 & 1300 & S. salamandra: $B$. calamita R. iberica R. perezi \\
\hline VL-0514 & VL-01 & 1380 & R. iberica; B. bufo \\
\hline VL-0916/0715/0816 & VL-01 & 1650 & B. bufo; B. calamita; Discoglossus; R. iberica \\
\hline VL-0011 & VL-01 & 1200 & S. salamandra; Discoglossus; B. calamita; R. iberica \\
\hline VL-0311 & VL-01 & 1250 & Discoglossus; H. arborea \\
\hline VL-0309 & VL-00 & 1360 & H. arborea; $R$. perezi \\
\hline UL-9808 & UL-90 & 1150 & $\begin{array}{l}\text { S. salamandra; Discoglossus; P. cultripes; B. bufo; } B \text {. } \\
\text { calamita; R. iberica }\end{array}$ \\
\hline UL-9806 & UL-90 & 1380 & S. salamandra; R. perezi; B. calamita \\
\hline UL-9805 & UL-90 & 1300 & S. salamandra; $B$. calamita \\
\hline UL-9906 & UL-90 & 1450 & B. calamita \\
\hline UL-9605 & UL-90 & 1700 & S. salamandra \\
\hline UL-9806 & UL-90 & 1390 & D. galganoi \\
\hline UL-9707 & UL-90 & 1360 & S. salamandra; $R$. perezi \\
\hline UK-8084 & UK- 88 & 820 & B. calamita \\
\hline UK-8183 & UK-88 & 830 & B. calamita \\
\hline UK-8482 & UK- 88 & 740 & L. boscai; $R$. perezi; A. cisternasii \\
\hline UK-8281 & UK-88 & 750 & B. calamita \\
\hline UK-8381 & UK-88 & 780 & B. calamita; A. cisternasii \\
\hline UK-8282 & UK-88 & 680 & H. arborea; B. calamita; T. pygmaeus \\
\hline UK-8385 & UK- 88 & 830 & T. pygmaeus; L. boscai; R. perezi; A. cisternasii; B. bufo \\
\hline UK-8586 & UK-88 & 920 & R. perezi \\
\hline UK-8486 & UK-88 & 880 & L. boscai; $R$. perezi \\
\hline UK-8585 & UK- 88 & 910 & D. galganoi; $R$. perezi \\
\hline UK-8785 & UK-88 & 880 & A. cisternasii; $R$. perezi \\
\hline UK-8785 & UK- 88 & 890 & R. perezi; L. boscai; T. pygmaeus; A. cisternasii; B. calamita \\
\hline VL-5110 & VL-51 & 800 & R. perezi \\
\hline VL-5210 & VL-51 & 700 & A. cisternasii; R. perezi \\
\hline VL-5407 & VL-50 & 640 & A. cisternasii; R. perezi \\
\hline VL-5110 & VL-51 & 800 & \\
\hline VL-5102 & VL-50 & 680 & \\
\hline VL-5308 & VL-50 & 700 & B. bufo \\
\hline VL-5407 & VL-50 & 640 & B. bufo \\
\hline UK-7474 & UK-77 & 660 & R. perezi \\
\hline UK-7573 & UK-77 & 640 & T. pygmaeus; P. cultripes; B. calamita; H. arborea; $R$. perezi \\
\hline UK-7574 & UK-77 & 630 & $\begin{array}{l}\text { T. pygmaeus; } H . \text { arborea; } R \text {. perezi; } P \text {. cultripes; } B \text {. } \\
\text { calamita; } P \text {. waltl }\end{array}$ \\
\hline
\end{tabular}




\begin{tabular}{|c|c|c|c|c|}
\hline El Tiemblo (AV) & UK-7574 & UK-77 & 640 & A. cisternasii; R. perezi \\
\hline El Tiemblo (AV) & UK-7169 & UK-76 & 1080 & R. perezi; L. boscai \\
\hline El Tiemblo (AV) & UK-7169 & UK-76 & 1090 & S. salamandra \\
\hline El Tiemblo (AV) & UK-7171 & UK-77 & 980 & S. salamandra \\
\hline El Tiemblo (AV) & UK-7168 & UK-76 & 1080 & R. iberica; R. perezi; S. salamandra; B. bufo; L. boscai \\
\hline El Tiemblo (AV) & UK-7272 & UK-77 & 880 & R. iberica; S. salamandra \\
\hline El Tiemblo (AV) & UK-7171 & UK-77 & 920 & \\
\hline El Tiemblo (AV) & UK-7370 & UK-77 & 1200 & \\
\hline El Tiemblo (AV) & UK-7270 & UK-77 & 1020 & \\
\hline El Tiemblo (AV) & UK-7269 & UK-76 & 1080 & L. boscai \\
\hline El Tiemblo (AV) & UK-7269 & UK-76 & 1160 & \\
\hline El Tiemblo (AV) & UK-7168 & UK-76 & 1100 & \\
\hline El Tiemblo (AV) & UK-7373 & UK-77 & 730 & R. perezi; L. boscai \\
\hline El Tiemblo (AV) & UK-7771 & UK-77 & 640 & \\
\hline El Tiemblo (AV) & UK-7874 & UK-77 & 640 & B. calamita; R. perezi \\
\hline El Tiemblo (AV) & UK-7771 & UK-77 & 640 & $\begin{array}{l}\text { L. boscai; R. perezi; D. galganoi; S. salamandra; B. bufo; } B \text {. } \\
\text { calamita }\end{array}$ \\
\hline El Vellón & VL-5513 & VL-51 & 680 & B. bufo \\
\hline El Vellón & VL-5012 & VL-51 & 850 & A. cisternasii; $B$. calamita \\
\hline El Vellón & VL-5111 & VL-51 & 790 & A. cisternasii; $R$. perezi \\
\hline El Vellón & VL-5013 & VL-51 & 890 & P. waltl; P. cultripes; B. calamita; $R$ perezi \\
\hline El Vellón & VL-5212 & VL-51 & 860 & B. calamita; $R$. perezi \\
\hline ElVellón & VL-5213 & VL-51 & 910 & R.perezi \\
\hline El Vellón & VL-5111 & VL-51 & 820 & B. bufo \\
\hline El Vellón & VL-5114 & VL-51 & 880 & R. perezi \\
\hline El Vellón & VL-5114 & VL-51 & 880 & B. bufo \\
\hline ElVellón & VL-5215 & VL-51 & 900 & \\
\hline El Viso de San Juan (TO) & VK-1346 & VK-14 & 570 & \\
\hline El Viso de San Juan (TO) & VK-1544 & VK-14 & 520 & D. galganoi; R. perezi \\
\hline Estremera & VK-9048 & VK-94 & 640 & P. punctatus; D. jeanneae \\
\hline Estremera & VK-9146 & VK-94 & 570 & \\
\hline Estremera & VK-9148 & VK-94 & 640 & B. calamita \\
\hline Estremera & VK-9243 & VK-94 & 560 & \\
\hline Fresnedilla (AV) & UK-6055 & UK-65 & 620 & B. calamita; H. meridionalis; $P$. cultripes \\
\hline Fresnedillas de la Oliva & VK-0080 & VK-08 & 780 & \\
\hline Fresnedillas de la Oliva & VK-0081 & VK-08 & 830 & B. calamita \\
\hline Fresnedillas de la Oliva & VK-0079 & VK-07 & 760 & B. bufo \\
\hline Fresnedillas de la Oliva & VK-0077 & VK-07 & 780 & P. cultripes; $B$. calamita \\
\hline Fresnedillas de la Oliva & VK-0082 & VK-08 & 910 & B. calamita \\
\hline Fresnedillas de la Oliva & VK-0184 & VK-08 & 890 & H. arborea \\
\hline Fresnedillas de la Oliva & UK-9983 & UK-98 & 960 & B. calamita \\
\hline Fresno de Torote & VK-6794 & VK-69 & 760 & B. calamita; R. perezi \\
\hline Fresno de Torote & VK-6495 & VK-69 & 700 & $\begin{array}{l}\text { A. cisternasii; D. jeanneae; } P \text {. waltl; B. calamita; } R \text {. perezi; } \\
\text { P. cultripes }\end{array}$ \\
\hline Fresno de Torote & VK-6593 & VK-69 & 650 & \\
\hline Fresno de Torote & VK-6593 & VK-69 & 650 & \\
\hline Fuenlabrada & VK-3457 & VK-35 & 620 & B. calamita \\
\hline Fuenlabrada & VK-3456 & VK-35 & 640 & \\
\hline Fuente el Saz de Jarama & VL-5200 & VL-50 & 620 & P. waltl; P. cultripes; B. calamita; R. perezi \\
\hline Fuente el Saz de Jarama & VK-5699 & VK-59 & 620 & \\
\hline Fuente el Saz de Jarama & VK-5596 & VK-59 & 620 & R.perezi; $P$. waltl \\
\hline Fuente el Saz de Jarama & VK-5797 & VK-59 & 640 & R.perezi \\
\hline Fuentidueña de Tajo & VK-8641 & VK-84 & 580 & \\
\hline Fuentidueña de Tajo & VK-8542 & VK-84 & 600 & \\
\hline Fuentidueña de Tajo & VK-8541 & VK-84 & 590 & B. calamita; $P$. punctatus; D. jeanneae; $R$. perezi \\
\hline Fuentidueña de Tajo & VK-8641 & VK-84 & 570 & \\
\hline Fuentidueña de Tajo & VK-8738 & VK-83 & 560 & R.perezi \\
\hline Fuentidueña de Tajo & VK-8640 & VK-84 & 560 & B. calamita \\
\hline Galapagar & VK-1588/1589 & VK-18 & 830 & P. cultripes; T. pygmaeus \\
\hline Galapagar & VK-1589 & VK-18 & 860 & B. calamita; $B$. bufo $; P$. walt $;$ P. cultripes $; R$. perezi \\
\hline Galapagar & VK-1688 & VK-18 & 860 & B. calamita; $R$. perezi \\
\hline Galapagar & VK-1594/1695 & VK-19 & 860 & $\begin{array}{l}\text { P. waltl; T. pygmaeus; } P \text {. cultripes; B. calamita; H. arborea; } \\
\text { R. perezi }\end{array}$ \\
\hline Galapagar & VK-1494 & VK-19 & 870 & \\
\hline Galapagar & VK-1493 & VK-19 & 880 & R. perezi; P. waltl; T. pygmaeus \\
\hline Galapagar & VK-1695 & VK-19 & 850 & \\
\hline Galapagar & VK-1690 & VK-19 & 900 & B. bufo; R. perezi \\
\hline Galapagar & VK-1590 & VK-19 & 870 & B. bufo; R. perezi \\
\hline Galapagar & VK-1793 & VK-19 & 860 & R. perezi \\
\hline Galapagar & VK-1790 & VK-19 & 880 & P. walt \\
\hline Galapagar & VK-1694/1695 & VK-19 & 830 & R. perezi; T. pygmaeus \\
\hline Galapagar & VK-1693 & VK-19 & 880 & \\
\hline Galapagar & VK-1594 & VK-19 & 870 & P. waltl \\
\hline Galapagar & VK-1791 & VK-19 & 830 & \\
\hline
\end{tabular}




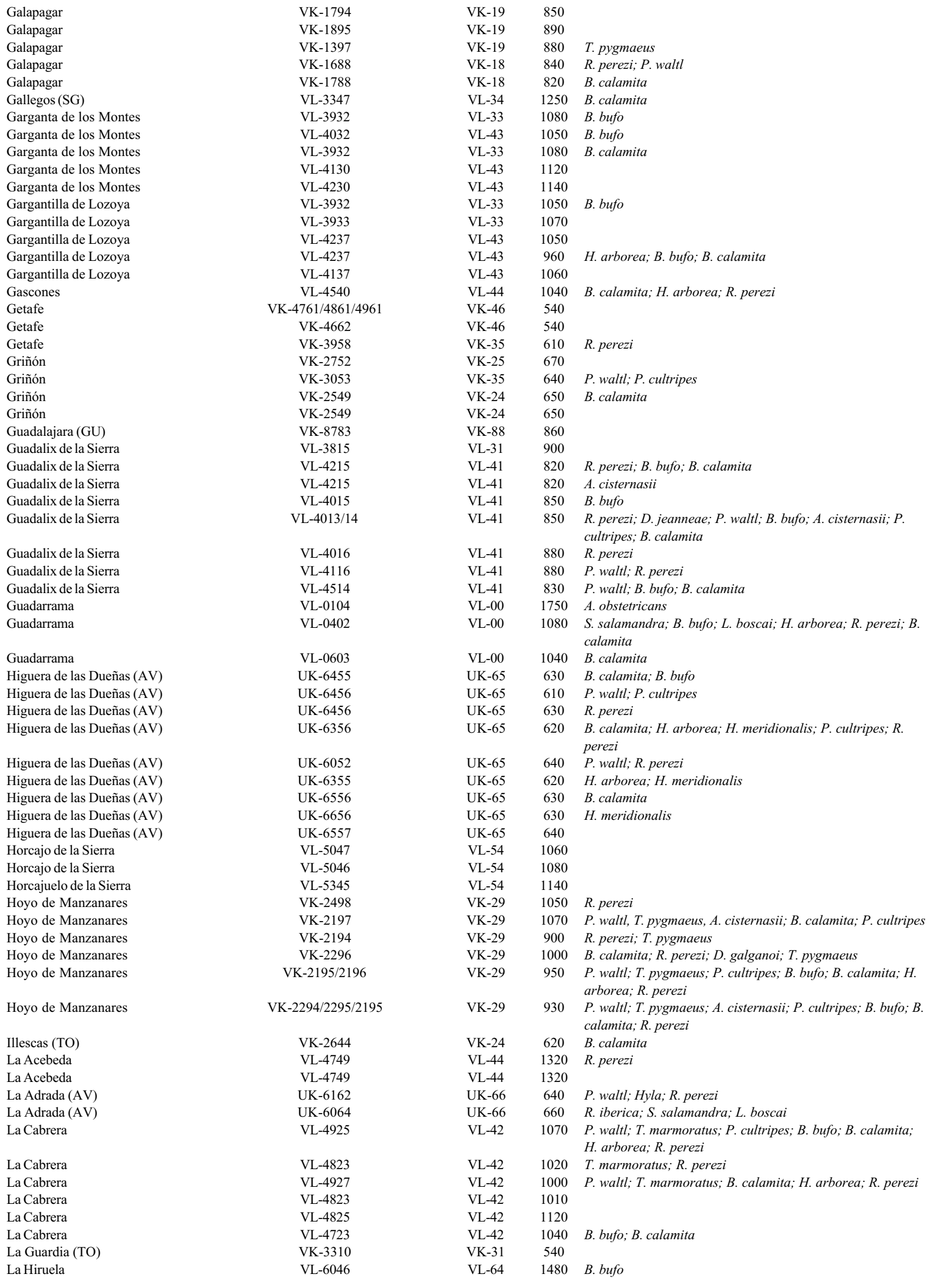


La Hiruela

La Serna del Monte

La Serna del Monte

La Serna del Monte

Las Navas del Marqués (AV)

Las Navas del Marqués (AV)

Las Navas del Marqués (AV)

Las Navas del Marqués (AV)

Las Navas del Marqués (AV)

Las Navas del Marqués (AV)

Las Navas del Marqués (AV)

Las Navas del Marqués (AV)

Las Navas del Marqués (AV)

Las Navas del Marqués (AV)

Las Navas del Marqués (AV)

Las Rozas de Madrid

Las Rozas de Madrid

Las Rozas de Madrid

Las Rozas de Madrid

Las Rozas de Puerto Real

Las Rozas de Puerto Real

Las Rozas de Puerto Real

Las Rozas de Puerto Real

Las Rozas de Puerto Real

Las Rozas de Puerto Real

Las Rozas de Puerto Real

Las Rozas de Puerto Real

Las Rozas de Puerto Real

Las Rozas de Puerto Real

Las Rozas de Puerto Real

Las Rozas de Puerto Real

Las Rozas de Puerto Real

Las Rozas de Puerto Real

Leganés

Leganés

Leganés

Leganés

Leganés

Leganés

Los Santos de la Humosa

Los Santos de la Humosa

Lozoya del Valle

Lozoya del Valle

Lozoya del Valle

Lozoya del Valle

Lozoya del Valle

Lozoya del Valle

Lozoya del Valle

Lozoya del Valle

Lozoya del Valle

Lozoya del Valle

Lozoya del Valle

Lozoyuela-Navas-Sieteiglesias

Lozoyuela-Navas-Sieteiglesias

Lozoyuela-Navas-Sieteiglesias

Lozoyuela-Navas-Sieteiglesias

Lozoyuela-Navas-Sieteiglesias

Lozoyuela-Navas-Sieteiglesias

Madarcos

Madarcos

Madrid

Madrid

Madrid

Madrid

Madrid

Madri

Madrid

Madric

Madric

Madrid

\begin{tabular}{|c|c|c|c|}
\hline VL-6348/6448 & VL-64 & 1120 & B. bufo \\
\hline VL-4742 & VL-44 & 1100 & B. bufo \\
\hline VL-4742 & VL-44 & 1080 & \\
\hline VL-4641 & VL-44 & 1040 & \\
\hline UK-8894 & UK-89 & 1100 & $\begin{array}{l}\text { S. salamandra; D. galganoi; B. calamita; H. arborea; } R \text {. } \\
\text { perezi; L. boscai }\end{array}$ \\
\hline UK-8797 & UK-89 & 1390 & R. perezi; H. arborea; $B$. calamita \\
\hline UK-8197 & UK-89 & 1350 & R. perezi; P. waltl; S. salamandra; B. calamita \\
\hline UK-8896 & UK-89 & 1400 & P. cultripes; B. bufo; R. iberica \\
\hline UK-8799 & UK-89 & 1420 & R. perezi \\
\hline UK-8796 & UK-89 & 1220 & \\
\hline UK-8995 & UK-89 & 1220 & \\
\hline UK-8791 & UK-89 & 1160 & B. calamita \\
\hline UK-8790/8890 & UK-89 & 1100 & R. perezi; B. bufo; L. boscai; T. pygmaeus; D. galganoi \\
\hline UK-8090 & UK-89 & 1250 & S. salamandra; B. bufo; B. calamita; H. arborea; L. boscai \\
\hline UK-9098 & UK-99 & 1320 & R. perezi \\
\hline VK-2491 & VK-29 & 720 & \\
\hline VK-2690 & VK-29 & 700 & \\
\hline VK-2087 & VK-28 & 640 & B. bufo \\
\hline VK-2184/2284 & VK-28 & 660 & P. waltl; P. cultripes; R. perezi; B. calamita \\
\hline UK-7260 & UK-76 & 870 & B. calamita; $R$. perezi \\
\hline UK-7262 & UK-76 & 900 & B. calamita; $R$. perezi \\
\hline UK-7362 & UK-76 & 840 & D. galganoi; R. perezi \\
\hline UK-7260 & UK-76 & 860 & B. calamita \\
\hline UK-7363 & UK-76 & 880 & \\
\hline UK-7263 & UK-76 & 840 & H. arborea; S. salamandra; R. perezi \\
\hline UK-7261 & UK-76 & 900 & Triturus; Bufo \\
\hline UK-7262 & UK-76 & 900 & \\
\hline UK-7262 & UK-76 & 950 & T. pygmaeus; Bufo \\
\hline UK-7063 & UK-76 & 820 & \\
\hline UK-7063 & UK-76 & 790 & S. salamandra; $R$. perezi \\
\hline UK-7163 & UK-76 & 830 & R.perezi \\
\hline UK-7058 & UK-75 & 750 & H. arborea; H. meridionalis; $B$. calamita \\
\hline UK-6858 & UK-65 & 660 & H. arborea; T. pygmaeus; P. waltl; P. cultripes; $R$. perezi \\
\hline VK-3567 & VK-36 & 630 & P. waltl \\
\hline VK-3165 & VK-36 & 680 & \\
\hline VK-3368 & VK-36 & 680 & B. calamita \\
\hline VK-3263 & VK-36 & 680 & B. calamita; $P$. cultripes; $P$. walt $;$ R. perezi \\
\hline VK3267 & VK-36 & 700 & B. calamita \\
\hline VK-3462 & VK-36 & 680 & \\
\hline VK-7784 & VK-78 & 720 & Discoglossus; P. punctatus; B. calamita; R. perezi \\
\hline VK-7883 & VK-78 & 890 & \\
\hline VL-3037 & VL-33 & 1960 & S. salamandra; $B$. calamita \\
\hline VL-2937 & VL-23 & 2100 & A. obstetricans \\
\hline VL-3132 & VL-33 & 1100 & H. arborea \\
\hline VL-3333 & VL-33 & 1120 & R. iberica \\
\hline VL-3434 & VL-33 & 1090 & B. bufo; B. calamita; Discoglossus; R. perezi \\
\hline VL-3136/37 & VL-33 & 1600 & S. salamandra; $R$. iberica \\
\hline VL-3235/36 & VL-33 & 1320 & R. iberica; R. perezi \\
\hline VL-3334 & VL-33 & 1170 & B. bufo \\
\hline VL-3236 & VL-33 & 1320 & B. bufo \\
\hline VL-3137 & VL-33 & 1740 & S. salamandra \\
\hline VL-3137 & VL-33 & 1730 & \\
\hline VL-5128 & VL-52 & 920 & A. cisternasii \\
\hline VL-4931 & VL-43 & 1020 & P. cultripes \\
\hline VL-4932/5032 & VL-43/53 & 980 & R. perezi; A. cisternasii; B. calamita \\
\hline VL-4530 & VL-43 & 1100 & R. perezi \\
\hline VL-4733 & VL-43 & 1040 & \\
\hline VL-4733 & VL-43 & 1030 & \\
\hline VL-5142 & VL-54 & 970 & B. bufo; A. cisternasii \\
\hline VL-5142 & VL-54 & 970 & R. perezi \\
\hline VK-3586 & VK-38 & 640 & B. calamita \\
\hline VK-3887 & VK-38 & 690 & P. waltl; P. cultripes; B. bufo; B. calamita; $R$. perezi \\
\hline VK-3692 & VK-39 & 650 & A. cisternasii; Bufo; R. perezi \\
\hline VK-3090 & VK-39 & 640 & \\
\hline VK-2893 & VK-29 & 680 & T. pygmaeus; A. cisternasii; B. bufo; R. perezi \\
\hline VK-2786 & VK-28 & 660 & A. cisternasii; $R$. perezi \\
\hline VK-2690 & VK-29 & 680 & R. perezi \\
\hline VK-3283 & VK-38 & 630 & P. cultripes; $B$. calamita \\
\hline VK-3783 & VK-38 & 680 & B. calamita; Discoglossus \\
\hline VK-3195 & VK-39 & 640 & R. perezi \\
\hline
\end{tabular}




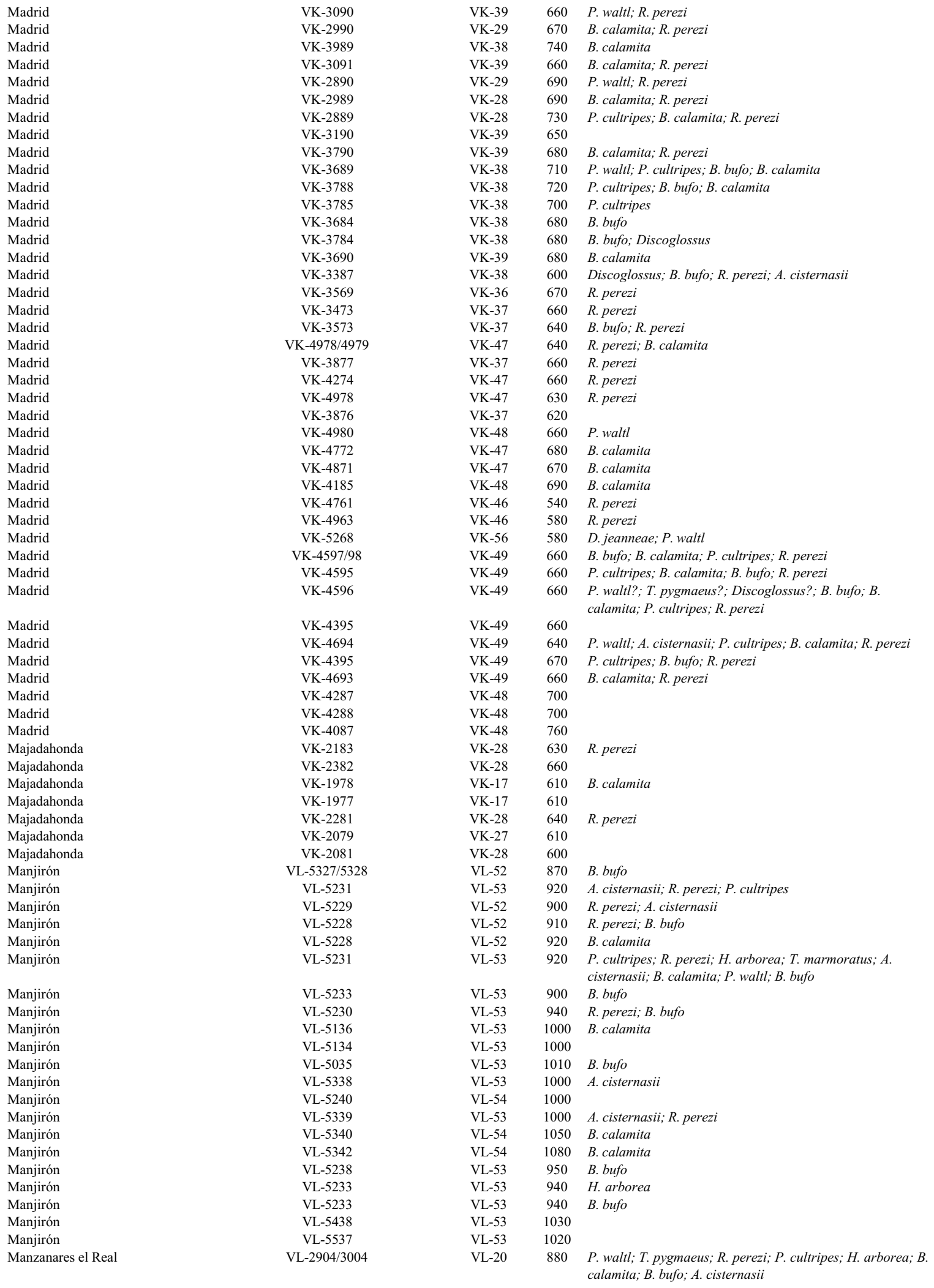




\begin{tabular}{|c|c|c|c|c|}
\hline Manzanares el Real & VL-2613 & VL-21 & 1270 & A. cisternasii; R. iberica \\
\hline Manzanares el Real & VL-2611 & VL-21 & 1300 & A. cisternasii; S. salamandra \\
\hline Manzanares el Real & VL-2914 & VL-21 & 1200 & \\
\hline Manzanares el Real & VL-2512 & VL-21 & 1120 & R. iberica \\
\hline Manzanares el Real & VL-2114 & VL-21 & 1530 & R. iberica \\
\hline Manzanares el Real & VL-3010 & VL-31 & 890 & T. pygmaeus \\
\hline Manzanares el Real & VL-2910 & VL-21 & 900 & A. cisternasii; H. arborea; $R$. perezi; B. calamita \\
\hline Manzanares el Real & VL-2508 & VL-20 & 920 & B. calamita; H. arborea; P. cultripes \\
\hline Manzanares el Real & VL-3012 & VL-31 & 990 & $\begin{array}{l}\text { R. perezi; } \text { T. pygmaeus; A. cisternasii; } D \text {. galganoi; } H \text {. } \\
\text { arborea; } P \text {. cultripes; } B \text {. bufo; } B \text {. calamita }\end{array}$ \\
\hline Manzanares el Real & VL-2910 & VL-21 & 890 & B. bufo; B. calamita; R. perezi; H. arborea \\
\hline Manzanares el Real & VL-2810 & VL-21 & 900 & \\
\hline Manzanares el Real & VL-2505 & VL-20 & 980 & B. bufo; R. perezi \\
\hline Manzanares el Real & VL-2606 & VL-20 & 980 & B. bufo \\
\hline Manzanares el Real & VL-2607 & VL-20 & 980 & P. cultripes \\
\hline Manzanares el Real & VL-2608 & VL-20 & 890 & T. pygmaeus; A. cisternasii; B. bufo; B. calamita; R. perezi \\
\hline Meco & VK-7290 & VK-79 & 670 & \\
\hline Meco & VK-7087 & VK-78 & 630 & R.perezi \\
\hline Meco & VK-7289 & VK-78 & 670 & P. punctatus; B. calamita; R. perezi \\
\hline Meco & VK-7188 & VK-78 & 640 & \\
\hline Meco & VK-7389 & VK-79 & 630 & \\
\hline Meco & VK-7189 & VK-78 & 660 & \\
\hline Meco & VK-7391 & VK-79 & 670 & \\
\hline Mejorada del Campo & VK-5972 & VK-57 & 590 & \\
\hline Mejorada del Campo & VK-5671 & VK-57 & 540 & \\
\hline Méntrida (TO) & UK-9859 & UK-95 & 500 & P. cultripes \\
\hline Méntrida (TO) & UK-9859 & UK-95 & 500 & P. cultripes \\
\hline Méntrida (TO) & UK-9854 & UK-95 & 560 & \\
\hline Méntrida (TO) & UK-9353 & UK-95 & 540 & R. perezi \\
\hline Méntrida (TO) & UK-9653 & UK-95 & 520 & B. calamita \\
\hline Méntrida (TO) & VK-0253 & VK-05 & 620 & B. calamita \\
\hline Méntrida (TO) & VK-0057 & VK-05 & 560 & B. bufo; B. calamita \\
\hline Méntrida (TO) & VK-0157 & VK-05 & 540 & B. bufo \\
\hline Méntrida (TO) & UK-9853 & UK-95 & 560 & \\
\hline Méntrida (TO) & UK-9954 & UK-95 & 580 & B. bufo \\
\hline Miraflores de la Sierra & VL-3519 & VL-31 & 1320 & \\
\hline Miraflores de la Sierra & VL-3317 & VL-31 & 1230 & B. calamita \\
\hline Miraflores de la Sierra & VL-3219 & VL-31 & 1300 & R. perezi; R. iberica; B. bufo; S. salamandra \\
\hline Miraflores de la Sierra & VL-3018 & VL-31 & 2120 & \\
\hline Miraflores de la Sierra & VL-3418 & VL-31 & 1180 & S. salamandra; B. bufo; R. iberica; R. perezi \\
\hline Miraflores de la Sierra & VL-3220 & VL-32 & 1430 & B. bufo \\
\hline Miraflores de la Sierra & VL-3220 & VL-32 & 1500 & B. bufo; R. iberica; S. salamandra \\
\hline Miraflores de la Sierra & VL-3414 & VL-31 & 1020 & H. arborea \\
\hline Miraflores de la Sierra & VL-3612 & VL-31 & 980 & $\begin{array}{l}\text { P. waltl; P. cultripes; R. perezi; H. arborea; D. galganoi; B. } \\
\text { calamita }\end{array}$ \\
\hline Mocejón (TO) & VK-2122 & VK-22 & 480 & R. perezi \\
\hline Mocejón (TO) & VK-2020 & VK-22 & 480 & R. perezi \\
\hline Mocejón (TO) & VK-2223 & VK-22 & 480 & R. perezi \\
\hline Mocejón (TO) & VK-2420 & VK-22 & 470 & \\
\hline Mocejón (TO) & VK-2219 & VK-21 & 480 & R. perezi \\
\hline Mocejón (TO) & VK-2122 & VK-22 & 480 & \\
\hline Mocejón (TO) & VK-2219 & VK-21 & 480 & R. perezi \\
\hline Mocejón (TO) & VK-2221 & VK-22 & 480 & \\
\hline Mocejón (TO) & VK-2319 & VK-21 & 460 & \\
\hline Mocejón (TO) & VK-2122 & VK-22 & 480 & \\
\hline Montejo de la Sierra & VL-5646 & VL-54 & 1140 & \\
\hline Montejo de la Sierra & VL-5546 & VL-54 & 1180 & Discoglossus \\
\hline Montejo de la Sierra & VL-5545 & VL-54 & 1100 & A. cisternasii \\
\hline Montejo de la Sierra & VL-5547/5647 & VL-54 & 1240 & B. bufo; R. perezi; P. cultripes \\
\hline Moraleja de Enmedio & VK-2357 & VK-25 & 620 & \\
\hline Moraleja de Enmedio & VK-2355 & VK-25 & 650 & \\
\hline Moraleja de Enmedio & VK-2557 & VK-25 & 650 & \\
\hline Morata de Tajuña & VK-6354 & VK-65 & 600 & \\
\hline Morata de Tajuña & VK-6355 & VK-65 & 690 & R.perezi \\
\hline Morata de Tajuña & VK-6653 & VK-65 & 670 & A. obstetricans; B. bufo; B. calamita \\
\hline Morata de Tajuña & VK-6252 & VK-65 & 580 & B. calamita \\
\hline Móstoles & VK-2164 & VK-26 & 580 & \\
\hline Móstoles & VK-2364 & VK-26 & 620 & \\
\hline Móstoles & VK-2264 & VK-26 & 620 & B. calamita; Discoglossus \\
\hline Móstoles & VK-2063 & VK-26 & 610 & \\
\hline Navacerrada & VL-1611 & VL-11 & 1380 & R.perezi \\
\hline Navacerrada & VL-1614 & VL-11 & 1700 & S. salamandra \\
\hline
\end{tabular}




\begin{tabular}{|c|c|c|c|c|}
\hline Navacerrada & VL-1613 & VL-11 & 1580 & S. salamandra \\
\hline Navacerrada & VL-1311 & VL-11 & 1380 & Discoglossus \\
\hline Navacerrada & VL-1515 & VL-11 & 1850 & S. salamandra; A. obstetricans; B. calamita \\
\hline Navafría (SG) & VL-3045/3144 & VL-34 & 1200 & B. bufo; B. calamita \\
\hline Navafría (SG) & VL-3041 & VL-34 & 1380 & \\
\hline Navafría (SG) & VL-2941 & VL-24 & 1360 & \\
\hline Navafría (SG) & VL-3241/42 & VL-34 & 1460 & B. bufo \\
\hline Navahondilla (AV) & UK-7465 & UK-76 & 690 & R. perezi; D. galganoi \\
\hline Navahondilla (AV) & UK-7364 & UK-76 & 700 & R.perezi \\
\hline Navahondilla (AV) & UK-7265 & UK-76 & 720 & \\
\hline Navahondilla (AV) & UK-7164 & UK-76 & 720 & R.perezi \\
\hline Navahondilla (AV) & UK-7365 & UK-76 & 700 & R. perezi \\
\hline Navalafuente & VL-4419 & VL-41 & 900 & B. bufo \\
\hline Navalafuente & VL-4218 & VL-41 & 920 & P. waltl; B. calamita; $R$. perezi \\
\hline Navalafuente & VL-4117 & VL-41 & 900 & B. calamita \\
\hline Navalafuente & VL-4218 & VL-41 & 930 & B. calamita; $P$. waltl; R. perezi \\
\hline Navalagamella & VK-0369 & VK-06 & 490 & D. galganoi; R. perezi; A. cisternasii \\
\hline Navalagamella & VK-0369 & VK-06 & 490 & $\begin{array}{l}\text { B. calamita; D. galganoi; } R \text {. perezi; } \text { A. cisternasii; } P \text {. } \\
\text { cultripes; } P \text {. waltl }\end{array}$ \\
\hline Navalagamella & VK-0078 & VK-07 & 740 & B. calamita \\
\hline Navalagamella & VK-0077 & VK-07 & 760 & $\begin{array}{l}\text { P. cultripes; B. calamita; } B \text {. bufo; } R \text {. perezi; D. galganoi; } A \text {. } \\
\text { cisternasii; T. pygmaeus; L. boscai }\end{array}$ \\
\hline Navalagamella & VK-0379 & VK-07 & 810 & $\begin{array}{l}\text { D. galganoi; } R \text {. perezi; L. boscai; T. pygmaeus; P. waltl; } P \text {. } \\
\text { cultripes; } B \text {. calamita }\end{array}$ \\
\hline Navalagamella & VK-0479 & VK-07 & 800 & D. galganoi; $B$. calamita $;$ P. cultripes \\
\hline Navalagamella & VK-0580 & VK-08 & 750 & \\
\hline Navalagamella & VK-0480 & VK-08 & 770 & \\
\hline Navalagamella & VK-0676 & VK-07 & 580 & R. perezi \\
\hline Navalagamella & VK-0775 & VK-07 & 540 & P. cultripes \\
\hline Navalagamella & VK-0580 & VK-08 & 730 & B. bufo \\
\hline Navalagamella & VK-0776 & VK-07 & 550 & B. calamita \\
\hline Navalagamella & VK-0681 & VK-08 & 730 & A. cisternasii; R. perezi \\
\hline Navalcarnero & VK-1861/1862 & VK-16 & 600 & \\
\hline Navalcarnero & VK-1662 & VK-16 & 610 & \\
\hline Navalcarnero & VK-1959 & VK-15 & 570 & \\
\hline Navalcarnero & VK-1959 & VK-15 & 560 & \\
\hline Navalcarnero & VK-1957 & VK-15 & 560 & R. perezi \\
\hline Navalcarnero & VK-1962 & VK-16 & 580 & \\
\hline Navalcarnero & VK-1761 & VK-16 & 610 & \\
\hline Navalcarnero & VK-1959 & VK-15 & 560 & \\
\hline Navalcarnero & VK-1856 & VK-15 & 560 & R. perezi \\
\hline Navalcarnero & VK-1762 & VK-16 & 620 & \\
\hline Navalcarnero & VK-1061 & VK-16 & 600 & \\
\hline Navalcarnero & VK-1961 & VK-16 & 580 & \\
\hline Navalcarnero & VK-1956 & VK-15 & 550 & \\
\hline Navalcarnero & VK-0862 & VK-06 & 570 & P. waltl \\
\hline Navarredonda & VL-3841 & VL-34 & 1650 & R. iberica \\
\hline Navarredonda & VL-3940 & VL-34 & 1300 & \\
\hline Navas del Rey & UK-9465 & UK-96 & 520 & R. perezi; B. bufo \\
\hline Navas del Rey & UK-8970 & UK-87 & 560 & B. calamita \\
\hline Navas del Rey & UK-8973 & UK-87 & 700 & \\
\hline Navas del Rey & UK-9471/72 & UK-97 & 700 & \\
\hline Navas del Rey & UK-9172 & UK-97 & 750 & \\
\hline Navas del Rey & UK-9071 & UK-97 & 580 & P. waltl; A. cisternasii; R. perezi \\
\hline Navas del Rey & UK-9471 & UK-97 & 690 & D. galganoi \\
\hline Navas del Rey & UK-9374 & UK-97 & 690 & A. cisternasii \\
\hline Nombela (TO) & UK-6851 & UK-65 & 860 & A. cisternasii; L. boscai \\
\hline Nuevo Baztán & VK-7766 & VK-76 & 830 & B. calamita \\
\hline Nuevo Baztán & VK-7668 & VK-76 & 820 & P. punctatus; B. calamita \\
\hline Nuevo Baztán & VK-7767 & VK-76 & 840 & R. perezi; B. calamita \\
\hline Nuevo Baztán & VK-7967 & VK-76 & 790 & \\
\hline Nuevo Baztán & VK-8070 & VK-87 & 770 & B. bufo \\
\hline Olmeda de las Fuentes & VK-8070 & VK-87 & 760 & P. punctatus; B. bufo; B. calamita; R. perezi \\
\hline Olmeda de las Fuentes & VK-8168 & VK-86 & 800 & A. obstetricans \\
\hline Olmeda de las Fuentes & VK-8168 & VK-86 & 800 & A. obstetricans \\
\hline Ontígola (TO) & VK-4827 & VK-42 & 590 & B. calamita \\
\hline Orusco & VK-8358 & VK-85 & 630 & \\
\hline Orusco & VK-8358 & VK-85 & 630 & \\
\hline Orusco & VK-8361 & VK-86 & 680 & D. jeanneae \\
\hline Orusco & VK-8360 & VK-86 & 640 & D. jeanneae \\
\hline Palazuelos de Eresma (SG) & VL-1129 & VL-12 & 1080 & T. marmoratus \\
\hline Paracuellos del Jarama & VK-5290/5390 & VK-59 & 590 & R. perezi \\
\hline Paracuellos del Jarama & VK-5289/5389 & VK-58 & 590 & R. perezi \\
\hline
\end{tabular}




\begin{tabular}{|c|c|c|c|c|}
\hline Paracuellos del Jarama & VK-5487 & VK-58 & 620 & \\
\hline Paracuellos del Jarama & VK-5586 & VK-58 & 660 & B. calamita \\
\hline Paracuellos del Jarama & VK-5782 & VK-58 & 630 & B. calamita \\
\hline Paredes de Escalona (TO) & UK-7852 & UK-75 & 500 & A. cisternasii; L. boscai; T. pygmaeus \\
\hline Paredes de Escalona (TO) & UK-7851 & UK-75 & 500 & D. galganoi; $P$. cultripes; H. meridionalis \\
\hline Paredes de Escalona (TO) & UK-8052 & UK-85 & 480 & D. galganoi; R. perezi; H. meridionalis \\
\hline Paredes de Escalona (TO) & UK-7951 & UK-75 & 480 & R. perezi; H. meridionalis \\
\hline Paredes de Escalona (TO) & UK-7654 & UK-75 & 660 & R. perezi; H. meridionalis \\
\hline Paredes de Escalona (TO) & UK-7752 & UK-75 & 580 & B. calamita \\
\hline Paredes de Escalona (TO) & UK-7453 & UK-75 & 640 & H. arborea; T. pygmaeus \\
\hline Paredes de Escalona (TO) & UK-7452 & UK-75 & 620 & H. meridionalis; T. pygmaeus \\
\hline Parla & VK-3355 & VK-35 & 660 & B. calamita \\
\hline Parla & VK-3154 & VK-35 & 650 & B. calamita \\
\hline Parla & VK-3252/3352/3452/3552/3551/3651 & VK-35 & 620 & R. perezi; B. calamita \\
\hline Parla & VK-3254 & VK-35 & 650 & P.walt \\
\hline Parla & VK-3254 & VK-35 & 650 & P. waltl; P. cultripes; B. calamita \\
\hline Parla & VK-3254 & VK-35 & 650 & P. waltl; P. cultripes \\
\hline Parla & VK-3851 & VK-35 & 610 & \\
\hline Parla & VK-3853 & VK-35 & 610 & R. perezi \\
\hline Parla & VK-3552 & VK-35 & 620 & B. calamita \\
\hline Parla & VK-3755 & VK-35 & 620 & P. waltl \\
\hline Patones & VL-6029 & VL-62 & 760 & R. perezi \\
\hline Patones & VL-5824 & VL-52 & 830 & \\
\hline Peguerinos (AV) & UK-9396 & UK-99 & 1340 & \\
\hline Peguerinos (AV) & UL-9801 & UL-90 & 1440 & B. bufo \\
\hline Peguerinos (AV) & UL-9700 & UL-90 & 1440 & R. perezi; R. iberica \\
\hline Peguerinos (AV) & UL-9800 & UL-90 & 1390 & R. iberica \\
\hline Peguerinos (AV) & UL-9803 & UL-90 & 1300 & R. iberica \\
\hline Peguerinos (AV) & UL-9800 & UL-90 & 1420 & R. iberica; R. perezi; S. salamandra \\
\hline Peguerinos (AV) & UL-9704 & UL-90 & 1500 & R. perezi \\
\hline Peguerinos (AV) & UK-9597 & UK-99 & 1360 & T. pygmaeus; R. perezi; H. arborea \\
\hline Peguerinos (AV) & UK-9998 & UK-99 & 1460 & B. calamita \\
\hline Peguerinos (AV) & UK-9899 & UK-99 & 1460 & \\
\hline Peguerinos (AV) & VL-0004 & VL-00 & 1600 & B. calamita \\
\hline Peguerinos (AV) & VK-0098 & VK-09 & 1540 & P. waltl \\
\hline Peguerinos (AV) & UK-9598 & UK-99 & 1350 & \\
\hline Peguerinos (AV) & UK-9496 & UK-99 & 1440 & \\
\hline Pelahustán (TO) & UK-6651 & UK-65 & 840 & R. perezi; A. cisternasii; L. boscai \\
\hline Pelahustán (TO) & UK-6450 & UK-65 & 770 & L. boscai; D. galganoi; B. bufo; B. calamita \\
\hline Pelahustán (TO) & UK-6451 & UK-65 & 760 & $\begin{array}{l}\text { A. cisternasii; L. boscai; T. pygmaeus; S. salamandra; } B \text {. } \\
\text { bufo; H. meridionalis }\end{array}$ \\
\hline Pelayos de la Presa & UK-8768 & UK-86 & 570 & \\
\hline Perales de Tajuña & VK-6753 & VK-65 & 580 & A. obstetricans; B. bufo \\
\hline Perales de Tajuña & VK-7054 & VK-75 & 600 & D. jeanneae; A. obstetricans \\
\hline Perales de Tajuña & VK-7054 & VK-75 & 650 & \\
\hline Perales de Tajuña & VK-7055 & VK-75 & 680 & A. obstetricans \\
\hline Perales de Tajuña & VK-7248 & VK-74 & 720 & A. obstetricans; D. jeanneae \\
\hline Pezuela de las Torres & VK- 8473 & VK-87 & & A. obstetricans \\
\hline Pezuela de las Torres & VK-8474 & VK-87 & 850 & P.punctatus \\
\hline Pezuela de las Torres & VK-8676 & VK-87 & 770 & \\
\hline Pinilla del Valle & VL-2937 & VL-23 & 2100 & A. obstetricans \\
\hline Pinilla del Valle & VL-2834 & VL-23 & 1500 & R. iberica \\
\hline Pinilla del Valle & VL-2936 & VL-23 & 1870 & S. salamandra; $B$. calamita \\
\hline Pinto & VK-4659 & VK-45 & 580 & B. bufo \\
\hline Piñuécar & VL-5041 & VL-54 & 1020 & B. bufo \\
\hline Piñuécar & VL-5042 & VL-54 & 1030 & \\
\hline Piñuécar & VL-5142 & VL-54 & 1000 & B. bufo \\
\hline Pioz (GU) & VK-8678 & VK-87 & 850 & P. punctatus; D. jeanneae \\
\hline Pozo de Guadalajara (GU) & VK-8582 & VK-88 & 880 & \\
\hline Pozo de Guadalajara (GU) & VK-8483 & VK-88 & 880 & B. calamita \\
\hline Pozuelo de Alarcón & VK-3072 & VK-37 & 700 & P. cultripes \\
\hline Pozuelo del Rey & VK-7167/7268 & VK-76 & 780 & P. cultripes; P. punctatus; R. perezi; $B$. calamita \\
\hline Pozuelo del Rey & VK-7268 & VK-76 & 800 & \\
\hline Pozuelo del Rey & VK-7368 & VK-76 & 810 & B. calamita; $P$. punctatus \\
\hline Pozuelo del Rey & VK-7269 & VK-76 & 810 & P. punctatus; D. jeanneae; B. calamita \\
\hline Prádena (SG) & VL-4059 & VL-45 & 1040 & \\
\hline Prádena (SG) & VL-4357 & VL-45 & 1150 & B. bufo \\
\hline Prádena (SG) & VL-4254 & VL-45 & 1120 & B. calamita \\
\hline Prádena del Rincón & VL-5544 & VL-54 & 1140 & B. bufo; R. perezi; H. arborea; $P$. cultripes \\
\hline Prádena del Rincón & VL-5644 & VL-54 & 1180 & B. bufo; H. arborea \\
\hline Prádena del Rincón & VL-5744 & VL-54 & 1230 & B. bufo; H. arborea \\
\hline
\end{tabular}




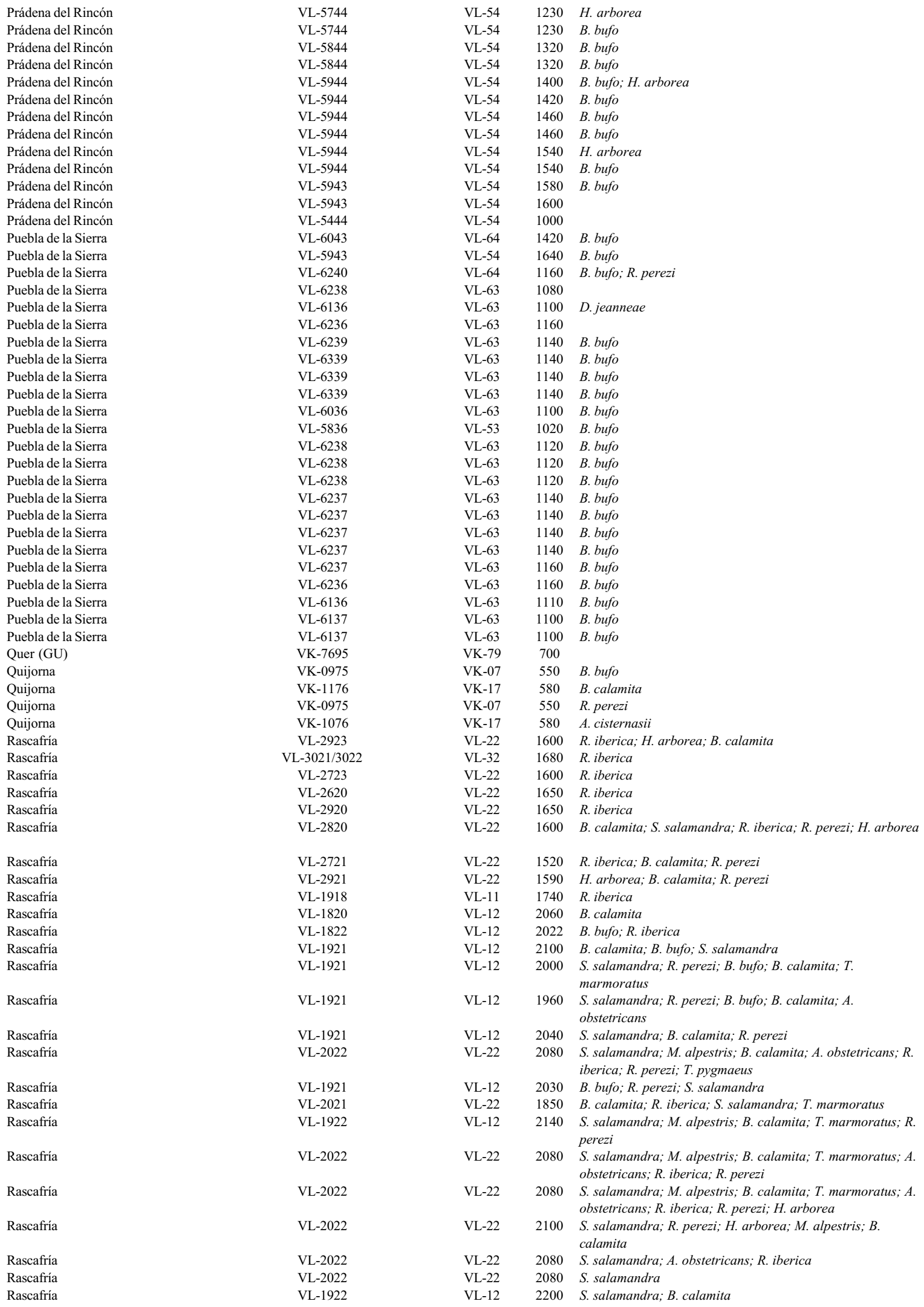




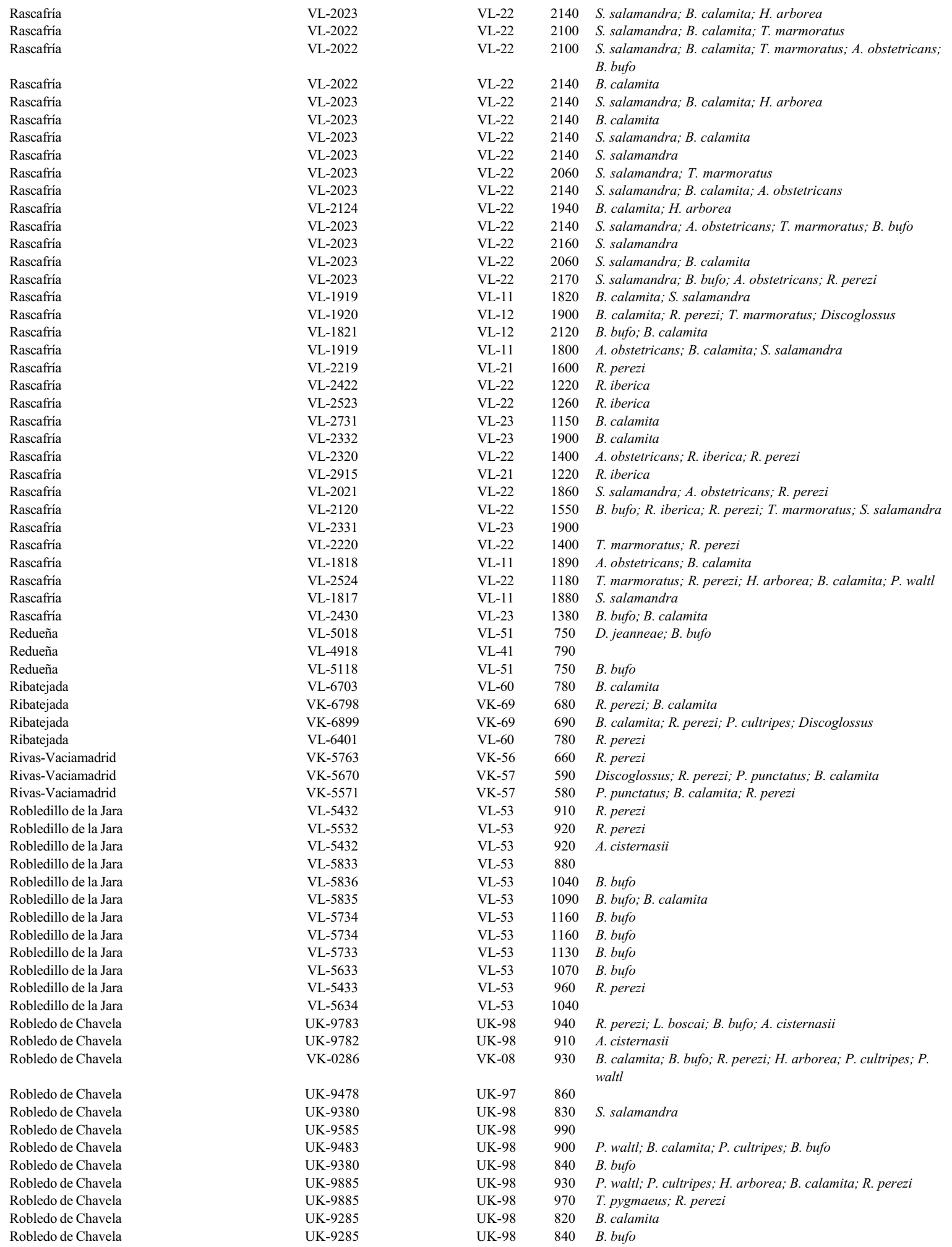


Robledo de Chavela

Robledo de Chavela

Robledo de Chavela

Robledo de Chavela

Robledondo

Robregordo

Robregordo

San Agustín de Guadalix

San Agustín de Guadalix

San Agustín de Guadalix

San Agustín de Guadalix

San Agustín de Guadalix

San Agustín de Guadalix

San Agustín de Guadalix

San Agustín de Guadalix

San Agustín de Guadalix

San Agustín de Guadalix

San Bartolomé de Pinares (AV)

San Bartolomé de Pinares (AV)

San Bartolomé de Pinares (AV)

San Bartolomé de Pinares (AV)

San Fernando de Henares

San Ildefonso o La Granja (SG)

San Ildefonso o La Granja (SG)

San Ildefonso o La Granja (SG)

San Ildefonso o La Granja (SG)

San Ildefonso o La Granja (SG)

San Ildefonso o La Granja (SG)

San Ildefonso o La Granja (SG)

San Ildefonso o La Granja (SG)

San Ildefonso o La Granja (SG)

San Ildefonso o La Granja (SG)

San Lorenzo de El Escorial

San Lorenzo de El Escorial

San Lorenzo de El Escorial

San Lorenzo de El Escorial

San Lorenzo de El Escorial

San Lorenzo de El Escorial

San Lorenzo de El Escorial

San Lorenzo de El Escorial

San Lorenzo de El Escorial

San Lorenzo de El Escorial

San Lorenzo de El Escorial

San Martín de la Vega

San Martín de la Vega

San Martín de la Vega

San Martín de la Vega

San Martín de la Vega

San Martín de la Vega

San Martín de la Vega

San Martín de la Vega

San Martín de la Vega

San Martín de Valdeiglesias

San Martín de Valdeiglesias

San Martín de Valdeiglesias

San Martín de Valdeiglesias

San Martín de Valdeiglesias

San Martín de Valdeiglesias

San Martín de Valdeiglesias

San Martín de Valdeiglesias

San Martín de Valdeiglesia

San Martín de Valdeiglesias

San Martín de Valdeiglesias

San Martín de Valdeiglesias

San Martín de Valdeiglesias

San Martín de Valdeiglesias

San Sebastián de los Reyes

San Sebastián de los Reyes

Santa Cruz de la Zarza (TO)
UK-9890

UK-9784

UK-9385

UK-9793

VL-5051

VL-5052

VK-4499

VL-4804

VL-4403

VL-4406

VL-4607

VL-4406

VL-4405

VL-4306

VL-4806

VL-4605

UK-8789

UK-8687/8787

UK- 8787

UK-8688

VK-5675

VL-1427

VL-1527

VL-1327

VL-1226/1326/1228

VL-1328

VL-1419/1519

VL-1819

VL-1023

VL-1425

VL-1322

VK-0194

VK-0195

VK-0295

VK-0194/0294

VK-0192

VK-0295

VK-0194

VK-0094

VK-0699

VL-1000

VK-0291

VK-5353

VK-4854

VK-4955

VK-4851

VK-5054

VK-4954

VK-5359

VK-5257

VK-5654

UK-7769

UK-7766

UK- 8365

UK-8567

UK-8466

UK-7870

UK-8372

UK-8272

UK-7968

UK- 817

UK-7767

UK-8068

UK-7869

UK-8067

VK-5292

VK-5191

VK-8134
UK-98 $\quad 840 \quad$ B. bufo

UK-98 1240

UK-98 $1000 \quad R$. perezi

UK-98 $\quad 820$

UK-99 1300 L. bosca

VL-55 1300

VL-55 $1365 \quad$ B. bufo; B. calamita

VK-49 $680 \quad$ R.perezi

VL-40 660

VL-40

VL-40

VL-40

VL-40

VL-40

VL-40

VL-40

VL-40

UK- 88

UK- 88

UK- -88

UK- 88

VK-57

VL-12

VL-12

VL-12

VL-12

VL-12

VL-11

VL-11

VL-12

VL-12

VL-12

VK-09

VK-09

VK-09

VK-09

VK-09

VK-09

VK-09

VK-09

VK-09

VL-10

VK-09

VK-55

VK-45

$\mathrm{VK}-45$

VK-45

VK-55

VK-45

$\mathrm{VK}-55$

VK-55

VK-55

UK-76

UK-76

UK-86

UK-86

UK-86

UK-77

UK- 87

UK- 87

UK-76

UK-87

640 B. calamita

850 B. calamita; $P$. waltl; R. perezi; B. bufo

$830 \quad$ B. calamita; $R$. perezi

850 B. calamita; P. cultripes

$800 \quad$ R. perezi

890 B. calamita

$660 \quad$ R. perezi; B. bufo

690 A. cisternasii; R. perezi

1060 B. bufo

960 H. arborea; L. boscai; D. galganoi; R. perezi

950 B. calamita

1000 L. boscai; R. perezi; D. galganoi; T. pygmaeus; B. calamita

$540 \quad$ R. perezi

1250 S. salamandra; T. marmoratus; A. obstetricans; B. bufo; $B$ calamita; H. arborea; R. iberica; R. perezi

1200 R. perezi; B. bufo; R. iberica; S. salamandra

1210 Discoglossus; B. calamita

1100 B. bufo; B. calamita

1190 T. marmoratus; Discoglossus; A. obstetricans; B. bufo; R. perezi

1420 S. salamandra; A. obstetrican

1800 A. obstetricans; $S$. salamandra

1260 R. iberica

1200 S. salamandra; B. bufo; B. calamita; R. iberica

1220 B. calamita; H. arborea

1300 P. waltl; S. salamandra; L. boscai; T. pygmaeus; R. iberica

1400 S. salamandra; P. waltl; T. pygmaeus; L. boscai; B.

calamita; $R$. perezi

1150 L. boscai; R. perezi; S. salamandra; P. waltl; B. bufo; T. pygmaeus; B. calamita

1300 S. salamandra

1320

1370 L. boscai; P. waltl; S. salamandra

960 H. arborea

$900 \quad$ B. bufo; B. calamita; R. perezi

1080 S. salamandra

510 Discoglossus; R. perezi

550

560

590 P. punctatus; B. calamita; R. perezi

520 B. calamita

560

20 R. perezi; B. calamita

560 P. punctatus

620 R. perezi

650 B. bufo; A. cisternasii; R. perezi

650 B. calamita

800 P. cultripes

670 B. calamita

800 S. salamandra; P. waltl; L. boscai; T. pygmaeus; R. perezi

630 L. boscai

600

610

$680 \quad$ R. perezi

$780 \quad$ L. boscai; $P$. waltl; R. perezi; T. pygmaeus; B. calamita; $P$. cultripes

UK-76 $650 \quad$ R. perezi; B. bufo; T. pygmaeus

UK-86 $680 \quad$ R. perezi; L. boscai; P. waltl; P. cultripes

UK-76

UK-86 760

VK-59 600

VK-59 $\quad 610$

VK-83 580 


\begin{tabular}{|c|c|c|c|c|}
\hline Santa María de la Alameda & UK-9995/9996 & UK-99 & 1420 & $\begin{array}{l}\text { S. salamandra; } R . \text { perezi; L. boscai; } T \text {. pygmaeus; } H \text {. } \\
\text { arborea; B. bufo; B. calamita; R. iberica; } P . \text { waltl }\end{array}$ \\
\hline Santa María de la Alameda & VK-0096/0197 & VK-09 & 1560 & R. perezi; B. bufo; S. salamandra \\
\hline Santa María de la Alameda & UK-9296 & UK-99 & 1160 & R. perezi; A. cisternasii \\
\hline Santa María de la Alameda & UK-9693 & UK-99 & 1260 & R. perezi \\
\hline Santa María de la Alameda & UK-9793 & UK-99 & 1300 & R. perezi \\
\hline Santa María de la Alameda & UK-9594 & UK-99 & 1180 & R. perezi \\
\hline Santa María de la Alameda & UK-9494 & UK-99 & 1300 & \\
\hline Santa María de la Alameda & UK-9392 & UK-99 & 1200 & B. calamita \\
\hline Santa María de la Alameda & UK-9391 & UK-99 & 1040 & R. perezi; B. bufo; A. cisternasii \\
\hline Santa María del Tiétar (AV) & UK-6864 & UK-66 & 940 & S. salamandra \\
\hline Santa María del Tiétar (AV) & UK-6764 & UK-66 & 1000 & P.waltl \\
\hline Santa María del Tiétar (AV) & UK-6962 & UK-66 & 700 & B. bufo; P. waltl; S. salamandra; B. calamita \\
\hline Santa María del Tiétar (AV) & UK-6860/6861 & UK-66 & 700 & $\begin{array}{l}\text { P. waltl; H. arborea; } H . \text { meridionalis; } R . \text { perezi; } S . \\
\text { salamandra; } L . \text { boscai; } T \text {. pygmaeus; } B . \text { calamita; } P . \\
\text { cultripes }\end{array}$ \\
\hline Santo Tomé del Puerto (SG) & VL-5059 & VL-55 & 1200 & P. waltl; Discoglossus; P. cultripes; H. arborea; R. perezi \\
\hline Santo Tomé del Puerto (SG) & VL-5158 & VL-55 & 1200 & R. perezi \\
\hline Santorcaz & VK-7981 & VK-78 & 790 & B. bufo; B. calamita; P. punctatus \\
\hline Santorcaz & VK-8281/8282 & VK-88 & 880 & $\begin{array}{l}\text { P. punctatus; B. calamita; P. waltl?; P. cultripes?; D. } \\
\text { jeanneae? }\end{array}$ \\
\hline Santorcaz & VK-8080 & VK-88 & 830 & \\
\hline Santorcaz & VK-8082 & VK-88 & 830 & B. calamita \\
\hline Santorcaz & VK-7982 & VK-78 & 790 & B. bufo \\
\hline Serranillos del Valle & VK-2350 & VK-25 & 620 & \\
\hline Seseña (TO) & VK-4038 & VK-43 & 580 & Discoglossus \\
\hline Seseña (TO) & VK-4438/4538 & VK-43 & 560 & \\
\hline Seseña (TO) & VK-4038 & VK-43 & 590 & P. punctatus; R. perezi; Discoglossus? \\
\hline Seseña (TO) & VK-4040 & VK-44 & 620 & R. perezi \\
\hline Seseña (TO) & VK-4535 & VK-43 & 480 & R. perezi \\
\hline Seseña (TO) & VK-3941 & VK-34 & 620 & \\
\hline Sevilla la Nueva & VK-1267 & VK-16 & 680 & B. calamita \\
\hline Somosierra & VL-5155 & VL-55 & 1400 & B. calamita \\
\hline Sotillo de la Adrada (AV) & UK-6659 & UK-65 & 650 & $\begin{array}{l}\text { P. cultripes; } P \text {. waltl; R. perezi; H. arborea; H. meridionalis; } \\
\text { B. calamita }\end{array}$ \\
\hline Sotillo de la Adrada (AV) & UK-6658 & UK-65 & 640 & $\begin{array}{l}\text { P. waltl; } P . \text { cultripes; } H . \text { meridionalis; } H . \text { arborea; } B . \\
\text { calamita; } \text { R. perezi }\end{array}$ \\
\hline Sotillo de la Adrada (AV) & UK-6558 & UK-65 & 640 & B. calamita; $R$. perezi; H. arborea \\
\hline Sotillo de la Adrada (AV) & UK-6662 & UK-66 & 660 & H. meridionalis \\
\hline Sotillo de la Adrada (AV) & UK-6461 & UK-66 & 640 & R. perezi; S. salamandra \\
\hline Sotillo de la Adrada (AV) & UK-6660 & UK-66 & 620 & R. perezi \\
\hline Sotillo de la Adrada (AV) & UK-6660/6760 & UK-66 & 640 & $\begin{array}{l}\text { B. calamita; D. galganoi; R. perezi; H. arborea; } H \text {. } \\
\text { meridionalis }\end{array}$ \\
\hline Soto del Real & VL-3110 & VL-31 & 900 & B. bufo \\
\hline Soto del Real & VL-3016 & VL-31 & 1300 & B. calamita \\
\hline Soto del Real & VL-3212 & VL-31 & 940 & B. bufo; B. calamita; D. galganoi; R. perezi; P. waltl \\
\hline Soto del Real & VL-3512 & VL-31 & 980 & $\begin{array}{l}\text { P. cultripes; B. bufo; B. calamita; } R \text {. perezi; } H \text {. arborea; } D \text {. } \\
\text { galganoi }\end{array}$ \\
\hline Soto del Real & VL-3512 & VL-31 & 980 & B. calamita; D. galganoi \\
\hline Soto del Real & VL-3412/13 & VL-31 & 940 & B. bufo \\
\hline Soto del Real & VL-3308 & VL-30 & 910 & \\
\hline Talamanca del Jarama & VL-6209 & VL-60 & 730 & R. perezi \\
\hline Talamanca del Jarama & VL-5707 & VL-50 & 660 & P. cultripes; A. cisternasii; R. perezi; B. calamita; P. waltl \\
\hline Talamanca del Jarama & VL-5714/5613/5614 & VL-51 & 670 & T. pygmaeus?; P. waltl; R. perezi; D. jeanneae \\
\hline Talamanca del Jarama & VL-5908/6008 & VL-50/60 & 700 & A. cisternasii; $P$. cultripes; $R$. perezi \\
\hline Tarancón (CU) & VK-9830 & VK-93 & 800 & D. jeanneae \\
\hline Tielmes & VK-7455 & VK-75 & 590 & B. bufo \\
\hline Tielmes & VK-7355 & VK-75 & 590 & A. obstetricans \\
\hline Tielmes & VK-7456 & VK-75 & 600 & D. jeanneae \\
\hline Tielmes & VK-7353 & VK-75 & 590 & A. obstetricans; D. jeanneae \\
\hline Tielmes & VK-7354 & VK-75 & 580 & A. obstetricans \\
\hline Tielmes & VK-7452 & VK-75 & 650 & A. obstetricans \\
\hline Toledo (TO) & VK-2616 & VK-21 & 480 & \\
\hline Toledo (TO) & VK-3721 & VK-32 & 530 & B. calamita \\
\hline Toledo (TO) & VK-2316 & VK-21 & 480 & \\
\hline Toledo (TO) & VK-2416 & VK-21 & 480 & B. calamita \\
\hline Toledo (TO) & VK-2216 & VK-21 & 480 & \\
\hline Toledo (TO) & VK-2515 & VK-21 & 500 & A. obstetricans; $R$. perezi \\
\hline Torrejón de Ardoz & VK-6176 & VK-67 & 570 & R. perezi \\
\hline Torrejón de Velasco & VK-3745 & VK-34 & 640 & \\
\hline Torrejón de Velasco & VK-3544 & VK-34 & 600 & \\
\hline Torrejón de Velasco & VK-3547 & VK-34 & 590 & \\
\hline Torrejón de Velasco & VK-3548 & VK-34 & 600 & \\
\hline Torrejón del Rey (GU) & VL-6900 & VL-60 & 690 & B. calamita; $P$. cultripes; $R$. perezi \\
\hline
\end{tabular}




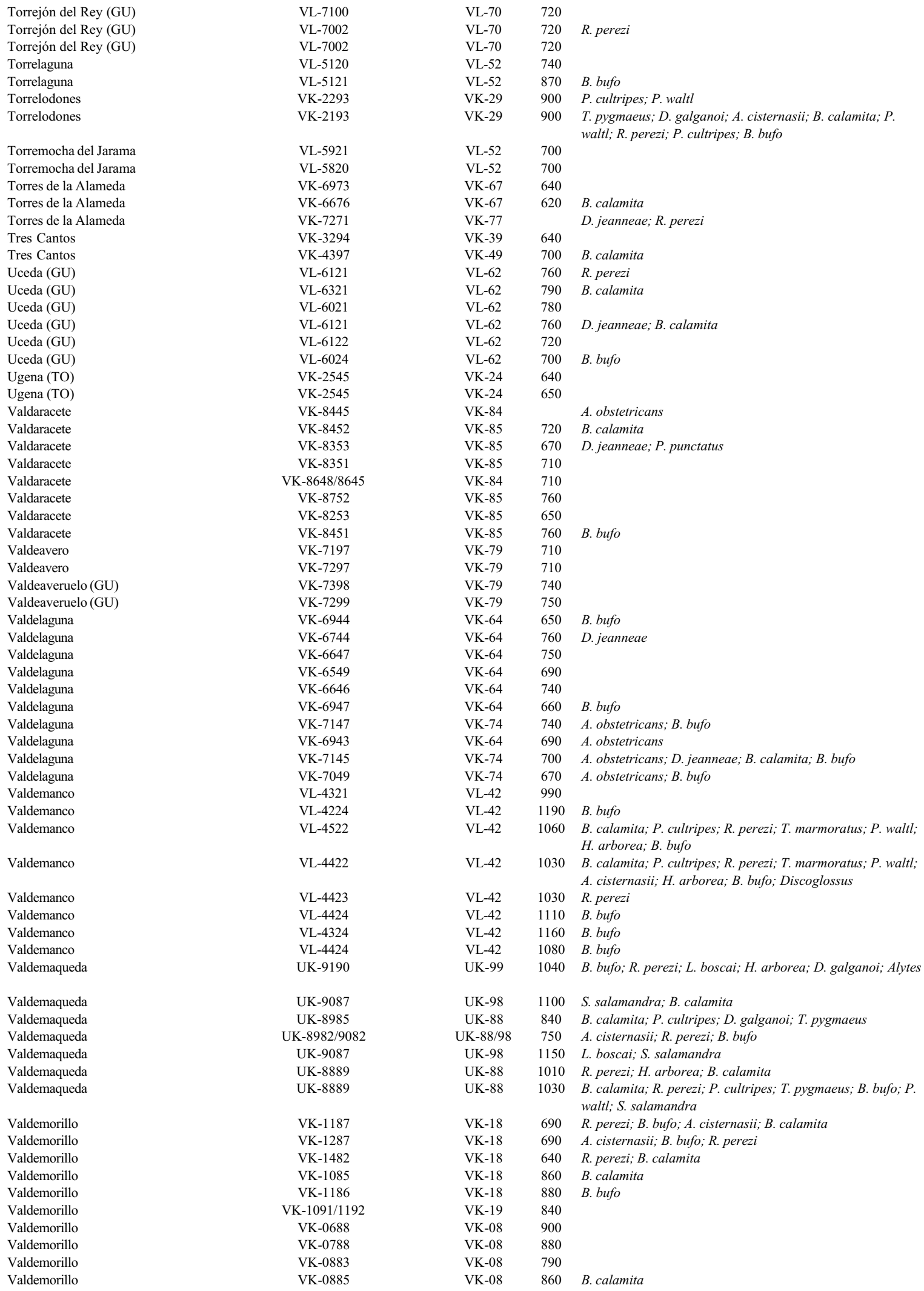




\begin{tabular}{|c|c|c|c|c|}
\hline Valdemorillo & VK-1285 & VK-18 & 880 & B. calamita \\
\hline Valdemorillo & VK-1084 & VK-18 & 820 & \\
\hline Valdemorillo & VK-0682 & VK-08 & 700 & A. cisternasii; H. arborea; R. perezi \\
\hline Valdemorillo & VK-0981 & VK-08 & 750 & B. bufo \\
\hline Valdemorillo & VK-0881 & VK-08 & 750 & B. bufo \\
\hline Valdemorillo & VK-0881 & VK-08 & 750 & B. bufo \\
\hline Valdemorillo & VK-1086 & VK-18 & 840 & H. arborea; B. calamita; $R$. perezi \\
\hline Valdemorillo & VK-1581 & VK-18 & 620 & \\
\hline Valdemoro & VK-4748 & VK-44 & 580 & \\
\hline Valdenuño-Fernández (GU) & VL-6913 & VL-61 & 870 & \\
\hline Valdenuño-Fernández (GU) & VL-6811 & VL-61 & 870 & \\
\hline Valdenuño-Fernández (GU) & VL-6913 & VL-61 & 890 & \\
\hline Valdeolmos & VK-6199 & VK-69 & 700 & \\
\hline Valdeolmos & VK-6198 & VK-69 & 690 & \\
\hline Valdepeñas de la Sierra (GU) & VL-6226 & VL-62 & 720 & R. perezi \\
\hline Valdepeñas de la Sierra (GU) & VL-6926 & VL-62 & 750 & \\
\hline Valdepeñas de la Sierra (GU) & VL-6428 & VL-62 & 800 & R. perezi \\
\hline Valdepeñas de la Sierra (GU) & VL-6331 & VL-63 & 910 & R. perezi \\
\hline Valdepeñas de la Sierra (GU) & VL-6827 & VL-62 & 800 & R.perezi \\
\hline Valdepeñas de la Sierra (GU) & VL-6729 & VL-62 & 890 & R. perezi; $P$. cultripes; $P$. waltl \\
\hline Valdepeñas de la Sierra (GU) & VL-6728 & VL-62 & 840 & R.perezi \\
\hline Valdepiélagos & VL-6212 & VL-61 & 820 & B. calamita; $P$. cultripes \\
\hline Valdepiélagos & VL-6112 & VL-61 & 780 & B. bufo \\
\hline Valdetorres de Jarama & VL-5903 & VL-50 & 690 & \\
\hline Valdilecha & VK-7458 & VK-75 & 620 & B. bufo; B. calamita \\
\hline Valdilecha & VK-7457 & VK-75 & 620 & B. calamita \\
\hline Valdilecha & VK-7457 & VK-75 & 610 & B. bufo \\
\hline Valdilecha & VK-7561 & VK-76 & 700 & D. jeanneae; P. punctatus; B. bufo \\
\hline Valmojado (TO) & VK-0651 & VK-05 & 660 & \\
\hline Valmojado (TO) & VK-0651 & VK-05 & 660 & \\
\hline Valverde de Alcalá & VK-7573 & VK-77 & 710 & \\
\hline Valverde de Alcalá & VK-7574 & VK-77 & 710 & B. bufo \\
\hline Valverde de Alcalá & VK-7473 & VK-77 & 750 & \\
\hline Velilla de San Antonio & VK-5967 & VK-56 & 540 & \\
\hline Velilla de San Antonio & VK-5769 & VK-56 & 550 & \\
\hline Velilla de San Antonio & VK-5966/6066 & VK-56/66 & 560 & R. perezi; B. calamita \\
\hline Velilla de San Antonio & VK-5868 & VK-56 & 550 & \\
\hline Villa del Prado & UK-9160 & UK-96 & 490 & B. calamita \\
\hline Villa del Prado & UK-9260 & UK-96 & 490 & B. bufo \\
\hline Villa del Prado & UK-8961 & UK-86 & 520 & A. cisternasii; R. perezi \\
\hline Villa del Prado & UK-9059 & UK-95 & 490 & R. perezi; A. cisternasii \\
\hline Villa del Prado & UK-8759 & UK-85 & 570 & \\
\hline Villa del Prado & UK-8659 & UK-85 & 570 & A. cisternasii; B. calamita; $R$. perezi; $P$. waltl \\
\hline Villa del Prado & UK-8659 & UK-85 & 600 & B. bufo \\
\hline Villa del Prado & UK-8958 & UK-85 & 500 & B. calamita \\
\hline Villa del Prado & UK-8957 & UK-85 & 490 & B. calamita \\
\hline Villa del Prado & UK-8956 & UK-85 & 480 & B. calamita \\
\hline Villa del Prado & UK-8955 & UK-85 & 480 & B. calamita \\
\hline Villa del Prado & UK-8854 & UK-85 & 480 & B. calamita; $P$. cultripes \\
\hline Villacastín(SG) & UL-8003 & UL-80 & 1250 & H. arborea \\
\hline Villaconejos & VK-5939 & VK-53 & 630 & B. calamita \\
\hline Villalbilla & VK-7177 & VK-77 & 660 & \\
\hline Villalbilla & VK-7275 & VK-77 & 790 & B. calamita; P. punctatus; $P$. cultripes \\
\hline Villamanrique de Tajo & VK-7836/7837 & VK-73 & 580 & A. obstetricans; B. calamita; R. perezi \\
\hline Villamanrique de Tajo & VK-7735 & VK-73 & 560 & B. calamita \\
\hline Villamanrique de Tajo & VK-8035 & VK-83 & 540 & B. calamita; $R$. perezi \\
\hline Villamanrique de Tajo & VK-7836 & VK-73 & 580 & R. perezi \\
\hline Villamanrique de Tajo & VK-7636 & VK-73 & 600 & \\
\hline Villamanta & VK-0361 & VK-06 & 520 & P. cultripes \\
\hline Villamanta & VK-0461 & VK-06 & 530 & P. cultripes \\
\hline Villamanta & VK-0361 & VK-06 & 520 & P.waltl \\
\hline Villamanta & VK-0663 & VK-06 & 580 & R. perezi; B. calamita \\
\hline Villamanta & VK-0461 & VK-06 & 550 & \\
\hline Villamanta & VK-0659 & VK-05 & 560 & $R$. perezi \\
\hline Villamanta & VK-0262 & VK-06 & 510 & P. cultripes \\
\hline Villamanta & VK-0262 & VK-06 & 510 & P. cultripes \\
\hline Villamanta & VK-0461 & VK-06 & 540 & B. calamita \\
\hline Villanueva de la Cañada & VK-1980/2080 & VK-18/28 & 610 & \\
\hline Villanueva de la Cañada & VK-1579/1679 & VK-17 & 630 & B. calamita; R. perezi \\
\hline Villanueva de la Cañada & VK-1477 & VK-17 & 640 & B. calamita \\
\hline Villanueva de la Cañada & VK-1977 & VK-17 & 620 & \\
\hline
\end{tabular}




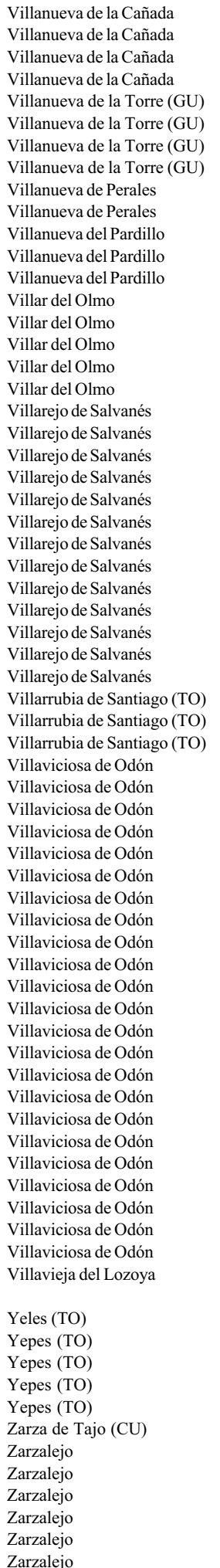

\begin{tabular}{|c|c|c|c|}
\hline VK-1777 & VK-17 & 650 & P. cultripes; B. calamita \\
\hline VK-1680 & VK-18 & 620 & R. perezi \\
\hline VK-1878 & VK-17 & 660 & \\
\hline VK-1978 & VK-17 & 600 & B. calamita; R. perezi; P. waltl; B. bufo \\
\hline VK-7591 & VK-79 & 670 & R.perezi \\
\hline VK-7493 & VK-79 & 710 & \\
\hline VK-7492 & VK-79 & 690 & \\
\hline VK-7492 & VK-79 & 700 & A. obstetricans \\
\hline VK-0666 & VK-06 & 580 & R.perezi \\
\hline VK-0670 & VK-07 & 580 & P. cultripes; B. calamita; R. perezi \\
\hline VK-2082 & VK-28 & 620 & B. bufo \\
\hline VK-2084 & VK-28 & 610 & \\
\hline VK-1884 & VK-18 & 660 & R. perezi; B. calamita; $P$. cultripes \\
\hline VK-8164 & VK-86 & 680 & B. bufo; Alytes? \\
\hline VK-8164 & VK-86 & 650 & B. bufo; B. calamita; P. punctatus \\
\hline VK-7964 & VK-76 & 770 & A. obstetricans \\
\hline VK-7965 & VK-76 & 690 & A. obstetricans \\
\hline VK-7965 & VK-76 & 820 & A. obstetricans \\
\hline VK-7340 & VK-74 & 700 & A. obstetricans; D. jeanneae; B. bufo \\
\hline VK-8237 & VK-83 & 540 & \\
\hline VK-8237 & VK-83 & 560 & R. perezi; B. calamita \\
\hline VK-8236 & VK-83 & 540 & R.perezi \\
\hline VK-8136 & VK-83 & 540 & B. calamita \\
\hline VK-7649 & VK-74 & 670 & D. jeanneae \\
\hline VK-7334 & VK-73 & 530 & A. obstetricans; B. calamita; R. perezi \\
\hline VK-8237 & VK-83 & 560 & B. bufo \\
\hline VK-7434 & VK-73 & 540 & P. punctatus \\
\hline VK-7747 & VK-74 & 740 & D. jeanneae \\
\hline VK-7348 & VK-74 & 770 & A. obstetricans \\
\hline VK-7749 & VK-74 & 710 & \\
\hline VK-7449 & VK-74 & 740 & B. bufo; D. jeanneae \\
\hline VK-7232 & VK-73 & 530 & A. obstetricans; R. perezi; B. calamita \\
\hline VK-7333 & VK-73 & 540 & R. perezi \\
\hline VK-7033 & VK-73 & 530 & P. punctatus; B. calamita; $R$. perezi \\
\hline VK-2173 & VK-27 & 640 & \\
\hline VK-1963 & VK-16 & 580 & \\
\hline VK-1869 & VK-16 & 620 & \\
\hline VK-1965 & VK-16 & 580 & R. perezi \\
\hline VK-1967/1767 & VK-16 & 580 & R. perezi; A. cisternasii \\
\hline VK-2267 & VK-26 & 630 & \\
\hline VK-2166 & VK-26 & 630 & B. calamita \\
\hline VK-1668 & VK-16 & 630 & B. calamita \\
\hline VK-2266 & VK-26 & 630 & B. calamita \\
\hline VK-1668 & VK-16 & 630 & B. calamita \\
\hline VK-1963 & VK-16 & 580 & B. calamita \\
\hline VK-1964 & VK-16 & 580 & B. bufo \\
\hline VK-1669 & VK-16 & 620 & \\
\hline VK-1768 & VK-16 & 640 & B. calamita \\
\hline VK-1868 & VK-16 & 630 & B. calamita \\
\hline VK-2166 & VK-26 & 650 & \\
\hline VK-1963 & VK-16 & 580 & Discoglossus \\
\hline VK-2068 & VK-26 & 580 & \\
\hline VK-1971/1970 & VK-17 & 580 & R.perezi \\
\hline VK-1863 & VK-16 & 630 & \\
\hline VK-1766 & VK-16 & 640 & B. calamita \\
\hline VK-2069 & VK-26 & 600 & \\
\hline VK-1963 & VK-16 & 570 & \\
\hline VL-4239 & VL-43 & 1080 & $\begin{array}{l}\text { P. waltl; Discoglossus; T. marmoratus; H. arborea; } R \text {. } \\
\text { perezi; B. calamita; P. cultripes }\end{array}$ \\
\hline VK-3141 & VK-34 & 550 & \\
\hline VK-3416 & VK-31 & 490 & R. perezi; A. obstetricans \\
\hline VK-3417 & VK-31 & 490 & \\
\hline VK-3317 & VK-31 & 490 & \\
\hline VK-3819/3918 & VK-31 & 620 & \\
\hline VK-8733 & VK-83 & 660 & \\
\hline VK-0089 & VK-08 & 1090 & S. salamandra \\
\hline VK-0189 & VK-08 & 1100 & S. salamandra \\
\hline VK-0288 & VK-08 & 990 & P. waltl; T. pygmaeus; P. cultripes \\
\hline VK-0288 & VK-08 & 1000 & B. calamita; R. perezi \\
\hline VK-0088 & VK-08 & 1000 & R.perezi \\
\hline UK-9990 & UK-99 & 1160 & P. waltl \\
\hline
\end{tabular}

